University of Tennessee Health Science Center

UTHSC Digital Commons

\title{
Vitamin D Levels Affect Survival in a BCR-ABL Acute Lymphoblastic Leukemia Mouse Model but Do Not Cause Vitamin-Drug Interactions
}

\author{
Kavya Annu \\ University of Tennessee Health Science Center
}

Follow this and additional works at: https://dc.uthsc.edu/dissertations

Part of the Chemical and Pharmacologic Phenomena Commons, Hemic and Lymphatic Diseases

Commons, Medical Nutrition Commons, Medical Pharmacology Commons, Medicinal and

Pharmaceutical Chemistry Commons, Nutritional and Metabolic Diseases Commons, Oncology

Commons, and the Other Pharmacy and Pharmaceutical Sciences Commons

\section{Recommended Citation}

Annu, Kavya (https://orcid.org/0000-0001-7312-2947), "Vitamin D Levels Affect Survival in a BCR-ABL Acute Lymphoblastic Leukemia Mouse Model but Do Not Cause Vitamin-Drug Interactions" (2020). Theses and Dissertations (ETD). Paper 534. http://dx.doi.org/10.21007/etd.cghs.2020.0523. 


\title{
Vitamin D Levels Affect Survival in a BCR-ABL Acute Lymphoblastic Leukemia Mouse Model but Do Not Cause Vitamin-Drug Interactions
}

\begin{abstract}
It is a well-established phenomenon that dietary components containing CYP3A inducers or inhibitors if co-administered with drugs that are CYP3A4 substrates lead to marked drug-drug interactions. Because vitamin $D$ is known to regulate intestinal CYP3A expression and gut CYP3A expression plays an important role in pre-systemic metabolism of CYP3A drugs, we determined the impact of vitamin D (VD3) status on systemic exposure and efficacy of chemotherapeutic agents that are CYP3A substrates. We employed VD3 sufficient and deficient mice to perform pharmacokinetics (PK) and anti-leukemic efficacy studies.

First, using hCYP3A4 transgenic mouse model we evaluated the intestinal, hepatic and renal expression of hCYP3A4, mouse CYPs and transporters in VD3 sufficient vs. deficient mice. We observed that female VD3 deficient mice had significantly lower duodenal mouse Cyp3a11 and hCYP3A4 expression than VD3 sufficient mice. Since intestinal CYP3A plays a significant role in first pass metabolism of drugs that are administered orally, chemotherapeutic agents such as dexamethasone (DEX) and dasatinib which are given orally to the patients were chosen to study the effect of VD3 status on systemic exposure of these CYP3A4 substrates.
\end{abstract}

In C57BL/6 mice, we performed dexamethasone PK study, where we observed male VD3 deficient mice had higher plasma DEX levels compared to sufficient mice on day 3.5. No significant differences were observed in females.

Another CYP3A4 substrate, dasatinib PK was also performed where AUC's were not significantly different between groups. However, during early absorption at $t=0.25 \mathrm{hr}$, VD3 deficient male mice had significantly higher dasatinib plasma levels compared to VD3 sufficient mice. In conclusion, VD3 levels (sufficient vs. deficient) significantly altered intestinal Cyp3a expression in female hCYP3A4 mice, and in male's, plasma exposure was different for orally administered DEX at 3.5 days, dasatinib at $0.25 \mathrm{hr}$. Ultimately, there was no significant effect of VD3 status on DEX or dasatinib PK indicating no potential vitamin drug interactions. However, DEX being a potential CYP3A inducer, we also observed significant induction of multiple mouse Cyp3a's in intestine of VD3 deficient but not VD3 sufficient mice. This differential induction of Cyp3a's due to vitamin D status could lead to drug interactions with other co-administered CYP3A substrates.

Given the high prevalence of VD3 deficiency in acute lymphoblastic leukemia (ALL) patients, we evaluated the effect of vitamin D deficiency on survival outcome from pre-B cell Arf -/- BCR-ABL acute lymphoblastic leukemia in a murine model. Vitamin D sufficient mice died earlier ( $p$

To investigate the mechanism by which vitamin $D$ affected the survival and disease growth in VD3 sufficient mice, we performed in vitro studies and demonstrated 1, 25-dihydroxy vitamin D (1, 25-(OH)2VD3) increased the number of BCR-ABL ALL cells only when co-cultured with bone marrow stroma. 1, 25-(OH)2VD3 induced CXCL12 production in vivo and in vitro in bone marrow stromal cells and CXCL12 increased stromal migration and the number of BCR-ABL blasts. Vitamin D together with ALL reprogrammed the marrow environment by increasing production of Type I and $V$ collagens, potentially trapping ALL blasts and CXCL12 expression to support tumor progression and leukemia cell homing.

In conclusion, although vitamin D deficiency did not cause significant interactions with anti-leukemic CYP3A4 substrates, there was a significant effect on disease progression and survival of mice from BCRABL ALL. 


\section{Document Type}

Dissertation

\section{Degree Name}

Doctor of Philosophy (PhD)

\section{Program}

Biomedical Sciences

\section{Research Advisor}

Erin G. Schuetz, PhD

\section{Keywords}

Acute lymphoblastic leukemia, CXCL12, CYP3A, Dasatinib, Dexamethasone, Vitamin D deficiency

\section{Subject Categories}

Chemical and Pharmacologic Phenomena | Diseases | Hemic and Lymphatic Diseases | Medical Nutrition | Medical Pharmacology | Medical Sciences | Medical Specialties | Medicinal and Pharmaceutical Chemistry | Medicine and Health Sciences | Nutritional and Metabolic Diseases | Oncology | Other Pharmacy and Pharmaceutical Sciences | Pharmacy and Pharmaceutical Sciences 
DOCTORAL DISSERTATION

\section{Vitamin D Levels Affect Survival in a BCR-ABL Acute Lymphoblastic Leukemia Mouse Model but Do Not Cause Vitamin-Drug Interactions}

Author:

Kavya Annu
Advisor:

Erin G. Schuetz, Ph.D.

A Dissertation Presented for The Graduate Studies Council of The University of Tennessee Health Science Center

in Partial Fulfillment of the Requirements for the Doctor of Philosophy degree from

The University of Tennessee

in

Integrated Biomedical Sciences: Molecular and Systems Pharmacology

College of Graduate Health Sciences

November 2020 
Copyright ( 92020 by Kavya Annu. All rights reserved. 


\section{DEDICATION}

To my beloved parents, husband, siblings and my friends.

I am and will forever be grateful for your unconditional love, patience and inspiration. 


\section{ACKNOWLEDGEMENTS}

With a number of people that made my journey not only effortless but also eventful and memorable, I would like to express my sincere gratitude to everyone that has been a part of my journey here at University of Tennessee Health Science Center and St. Jude Children's Research Hospital.

Firstly, I would like to express my deepest gratitude and profound appreciation to my mentor Dr. Erin G. Schuetz for believing in me and providing me this opportunity for doing my doctoral research in her laboratory. Dr. Schuetz is a wonderful mentor and has been very supportive in my successful completion of my doctoral degree. Your insightful feedback during our discussions have pushed me further to sharpen my thinking and made my work better. I would like to express my sincere thanks to my dissertation committee members, Dr. Taosheng Chen, Dr. Elizabeth A. Fitzpatrick, Dr. Santosh Kumar, and Dr. Alex Sparreboom who have been instrumental in directing my project towards the right direction. Your guidance and suggestions were very invaluable to conduct my doctoral research.

I would like to acknowledge my previous and current lab mates for their wonderful attitude towards me and being helpful to take my research further. Dr. Yasuda Kazuto and Dr. Samit Ganguly for providing me the hands-on experience with different techniques and making my time here more productive. My special thanks to Dr.

Kamalika Mukherjee who has been very supportive, encouraging me professionally and also has become a personal friend. I would also like to thank Dr. Jingjun Lu, Dr. Amarjit Choudhry, Nick Gerard, Shweta Hosagoudar for being supportive and helpful during my $\mathrm{PhD}$ training. My sincere thanks to Dr. John Schuetz and his lab members for their suggestions and support. A special and heartfelt thanks to my dear friend Mahesh C Kodali, whose support and encouragement got me here.

I am very much grateful to Department of Pharmaceutical Sciences, St. Jude Children's Research Hospital for being very supportive and all the administrative staff for being helpful whenever I needed them. I would like to express my gratitude to administrative staff at Integrated Biomedical Sciences program at College of Graduate Health Sciences, UTHSC.

Finally, I express my heartfelt gratitude to my parents and siblings who supported me throughout my academic career and are always my strength. Unconditional love, support and encouragement from my husband Surya Kothapally has motivated me every time I needed it. I would also like to thank my in-laws and friends for supporting me. 


\begin{abstract}
It is a well-established phenomenon that dietary components containing CYP3A inducers or inhibitors if co-administered with drugs that are CYP3A4 substrates lead to marked drug-drug interactions. Because vitamin D is known to regulate intestinal CYP3A expression and gut CYP3A expression plays an important role in pre-systemic metabolism of CYP3A drugs, we determined the impact of vitamin D (VD 3 ) status on systemic exposure and efficacy of chemotherapeutic agents that are CYP3A substrates. We employed VD3-sufficient and -deficient mice to perform pharmacokinetics (PK) and anti-leukemic efficacy studies.

First, using hCYP3A4 transgenic mouse model we evaluated the intestinal, hepatic and renal expression of hCYP3A4, mouse CYPs and transporters in $\mathrm{VD}_{3}$ sufficient versus $\mathrm{VD}_{3}$ deficient mice. We observed that female $\mathrm{VD}_{3}$ deficient mice had significantly lower duodenal mouse Cyp3a11 and hCYP3A4 expression than $\mathrm{VD}_{3}$ sufficient mice. Since intestinal CYP3A plays a significant role in first pass metabolism of drugs that are administered orally, chemotherapeutic agents such as dexamethasone (DEX) and dasatinib which are given orally to the patients were chosen to study the effect of $\mathrm{VD}_{3}$ status on systemic exposure of these CYP3A4 substrates.
\end{abstract}

In C57BL/6 mice, we performed DEX PK study, where we observed male VD3 deficient mice had higher plasma DEX levels compared to sufficient mice on day 3.5. No significant differences were observed in females.

Another CYP3A4 substrate, dasatinib PK was also performed where AUC's were not significantly different between groups. However, during early absorption at $t=0.25$ $\mathrm{hr}, \mathrm{VD}_{3}$ deficient male mice had significantly higher dasatinib plasma levels compared to $\mathrm{VD}_{3}$ sufficient mice. In conclusion, $\mathrm{VD}_{3}$ levels (sufficient versus deficient) significantly altered intestinal Cyp3a expression in female hCYP3A4 mice, and in male's, plasma exposure was different for orally administered DEX at 3.5 days, dasatinib at $0.25 \mathrm{hr}$. Ultimately, there was no significant effect of VD3 status on DEX or dasatinib PK indicating no potential vitamin drug interactions. However, DEX being a potential CYP3A inducer, we also observed significant induction of multiple mouse Cyp3a's in intestine of $\mathrm{VD}_{3}$ deficient but not VD3 sufficient mice. This differential induction of Cyp3a's due to vitamin D status could lead to drug interactions with other coadministered CYP3A substrates.

Given the high prevalence of $\mathrm{VD}_{3}$ deficiency in acute lymphoblastic leukemia (ALL) patients, we evaluated the effect of vitamin D deficiency on survival outcome from pre-B cell $\mathrm{Arf}{ }^{-/-}$BCR-ABL acute lymphoblastic leukemia in a murine model. Vitamin D sufficient mice died earlier $(\mathrm{p}<0.003)$ compared to vitamin D deficient mice.

To investigate the mechanism by which vitamin $\mathrm{D}$ affected the survival and disease growth in $\mathrm{VD}_{3}$ sufficient mice, we performed in vitro studies and demonstrated 1 , 25-dihydroxy vitamin $\mathrm{D}\left(1,25-(\mathrm{OH})_{2} \mathrm{VD}_{3}\right)$ increased the number of BCR-ABL ALL cells 
only when co-cultured with bone marrow stroma. 1, 25-(OH $)_{2} \mathrm{VD}_{3}$ induced CXCL12 production in vivo and in vitro in bone marrow stromal cells and CXCL12 increased stromal migration and the number of BCR-ABL blasts. Vitamin D together with ALL reprogrammed the marrow environment by increasing production of Type I and V collagens, potentially trapping ALL blasts and CXCL12 expression to support tumor progression and leukemia cell homing.

In conclusion, although vitamin D deficiency did not cause significant interactions with anti-leukemic CYP3A4 substrates, there was a significant effect on disease progression and survival of mice from BCR-ABL ALL. 


\section{TABLE OF CONTENTS}

CHAPTER 1. INTRODUCTION ..........................................................................................1

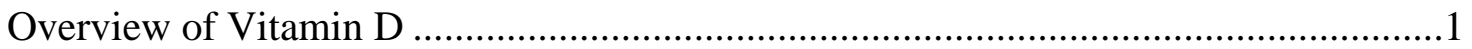

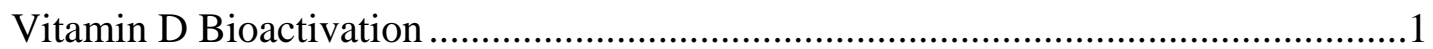

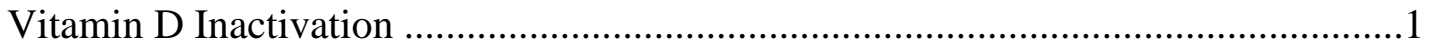

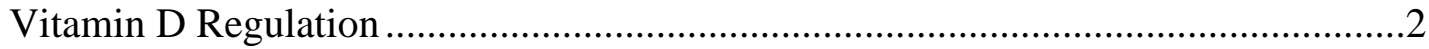

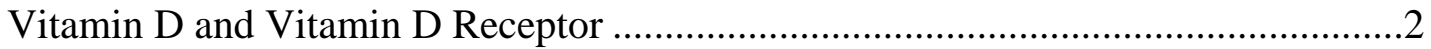

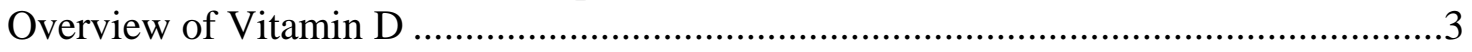

CYP3A4/Cyp3a Gene ........................................................................................

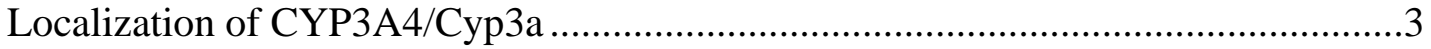

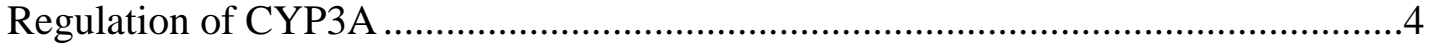

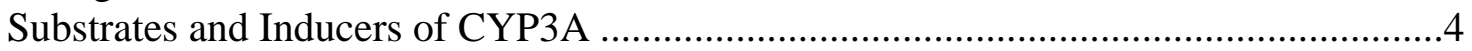

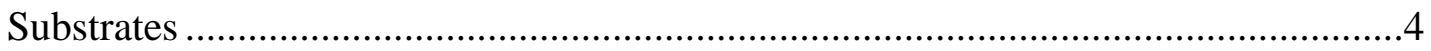

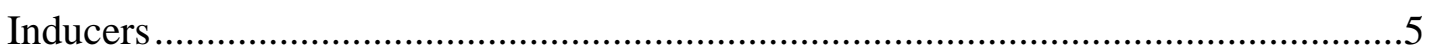

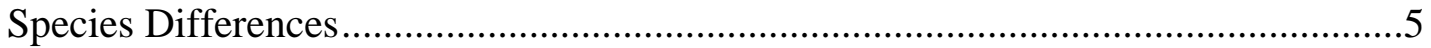

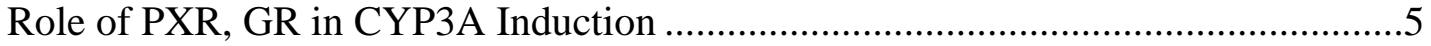

Dietary Interactions with CYP3A ...............................................................6

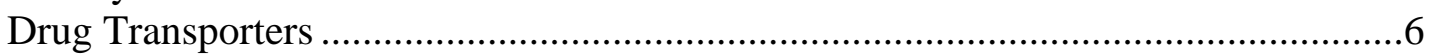

Vitamin D Deficiency ..............................................................................

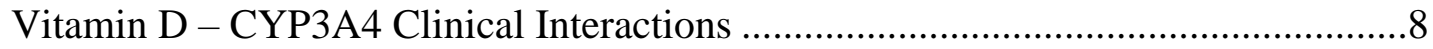

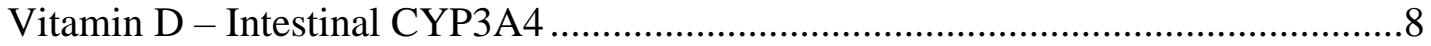

CHAPTER 2. VITAMIN D AND LEUKEMIA ........................................................10

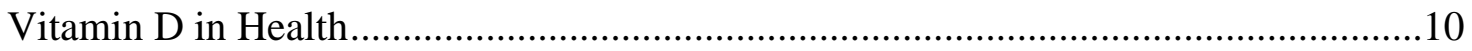

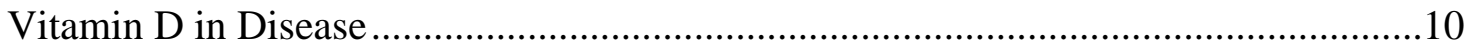

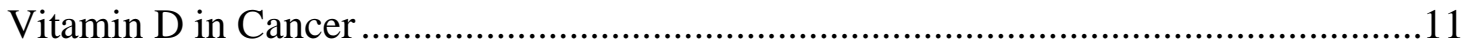

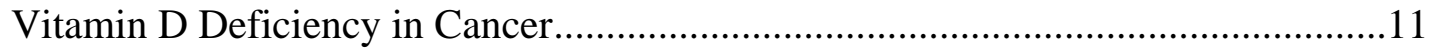

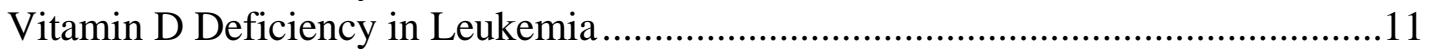

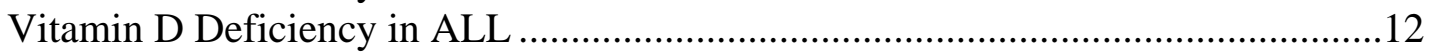

Role of Vitamin D in the Bone Marrow Microenvironment .......................................13

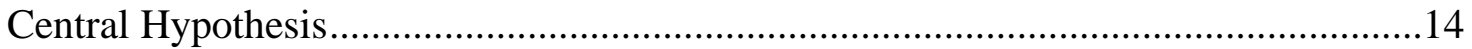

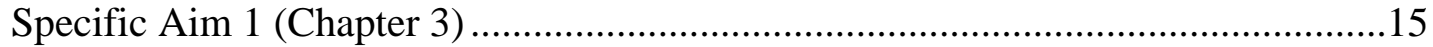

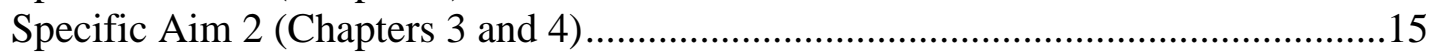

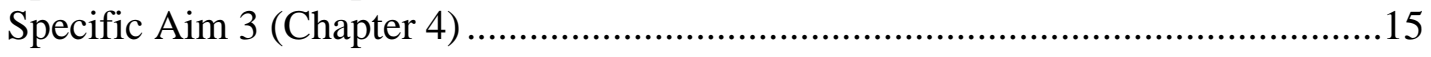

\section{CHAPTER 3. VITAMIN D STATUS ALTERS DRUG DETOXIFICATION GENE EXPRESSION AND DEXAMETHASONE INDUCTION OF

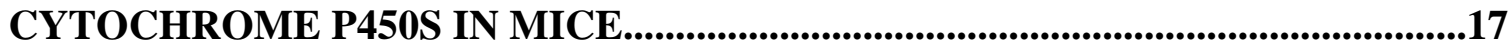

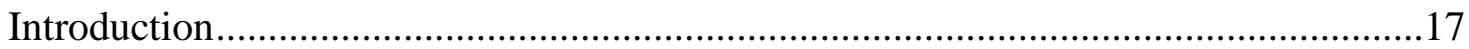

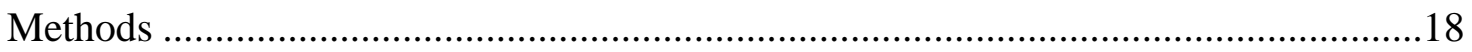

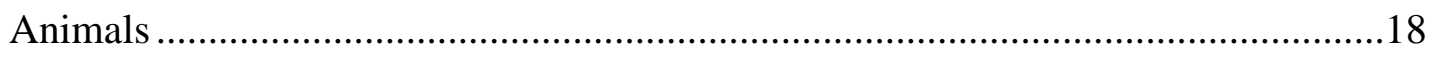

mRNA Quantification by Real-time PCR .......................................................18

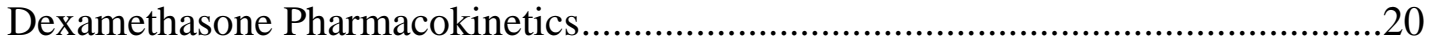

Dexamethasone administered in drinking water .......................................... 20 


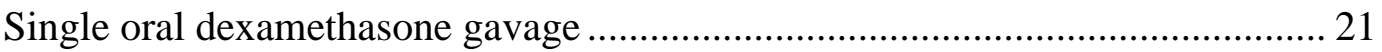

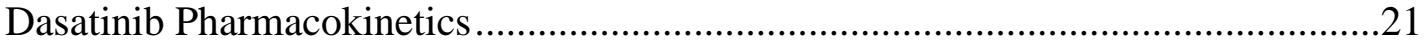

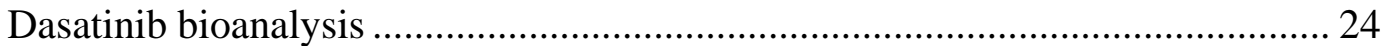

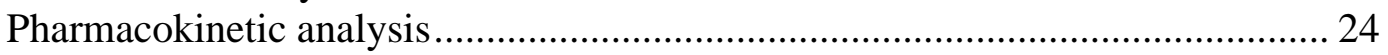

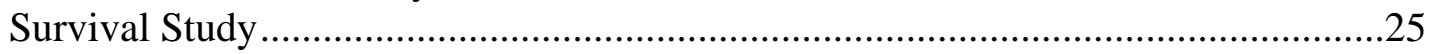

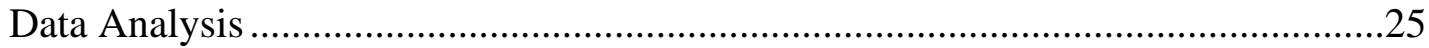

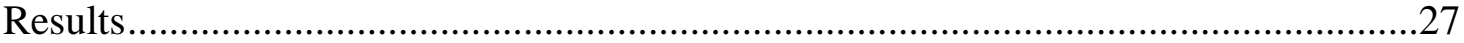

Impact of Vitamin D Deficiency on Murine Duodenum Cyp3a and Transporter

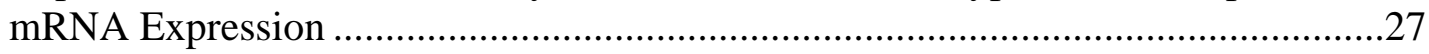

Cyp and Transporter Expression in Liver and Kidney of VD3-Sufficient and -

Deficient hCYP3A4-tg Mice …………………………..........................................27

Plasma Dexamethasone Concentrations in VD3 3 Deficient versus Sufficient

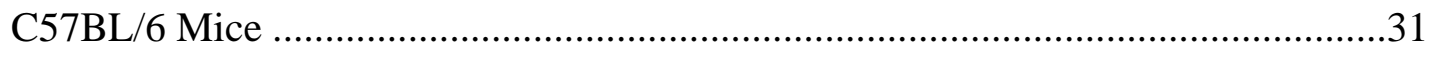

Tissue-specific Impact of Dexamethasone on Intestinal and Hepatic CYP

Expression in C57BL/6 VD 3 Sufficient versus VD 3 Deficient Mice...........................31

Dasatinib Pharmacokinetics (Oral Gavage) in C57BL/6 Mice....................................35

Effect of Vitamin D Status on Anti-leukemic Efficacy of Dexamethasone ...............35

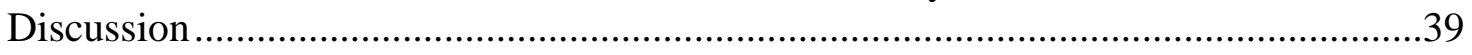

\section{CHAPTER 4. ROLE OF VITAMIN D IN BCR-ABL ARF-/- ACUTE}

LYMPHOBLASTIC LEUKEMIA ........................................................................46

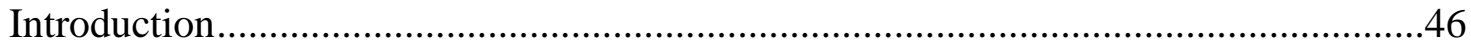

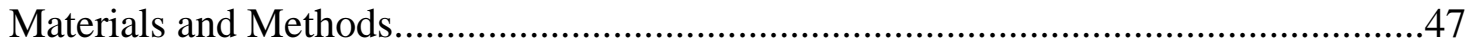

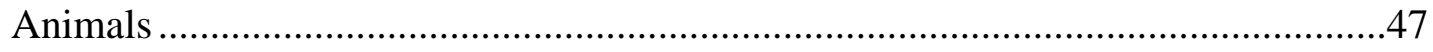

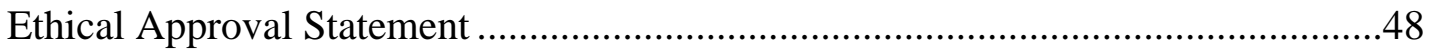

Leukemia and Bone Marrow Stroma Cells ............................................................48

BCR-ABL ALL Disease Generation and Monitoring ……….................................48

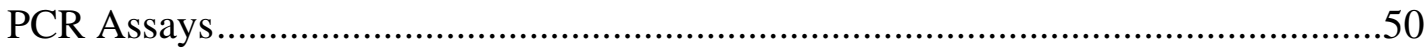

PCR to determine gender of BCR-ABL ALL cells ............................................. 50

PCR to detect VDR mRNA ……………………….................................... 51

Analysis of in vivo Growth of Leukemic Burden .....................................................51

BCR-ABL ALL Viable Cell Number Assay …………….......................................52

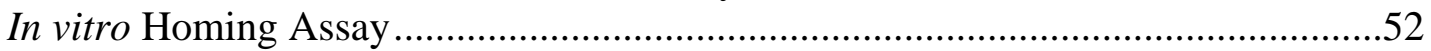

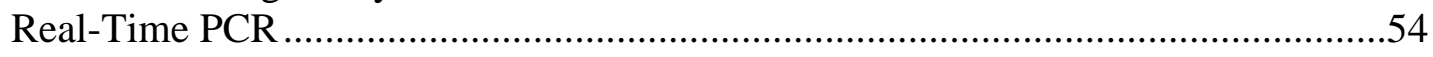

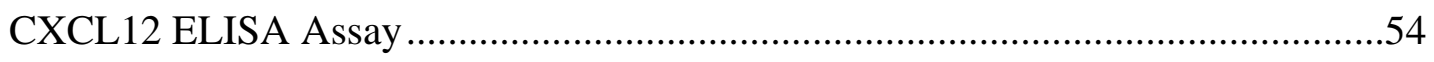

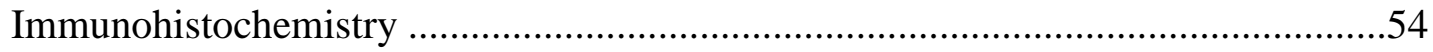

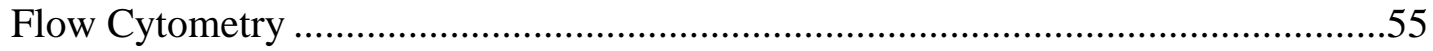

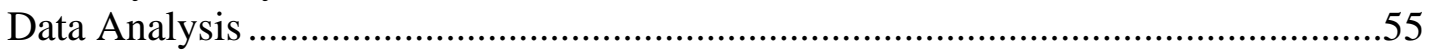

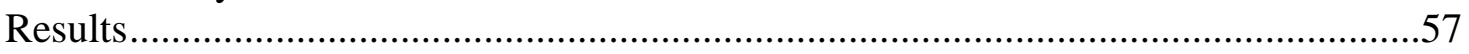

Vitamin D Deficiency Improves Survival of Mice with BCR-ABL Arf-/- B-ALL ..57

Bone Marrow and Spleen BCR-ABL Leukemia Burden is Lower in VDD Mice

Compared to Vitamin D Sufficient Mice.................................................................64

Mice Vitamin D Deficient for a Shorter Time Interval Still Show Increased

Survival from BCR-ABL ALL Compared to Vitamin D-Sufficient Mice ..................64

$1,25(\mathrm{OH})_{2} \mathrm{VD}_{3}$ Increases the Number of BCR-ABL Arf-/- ALL Cells Only

When Co-cultured with Bone Marrow Stromal Cells. 
1, 25(OH) $)_{2} \mathrm{VD}_{3}$ Increases BCR-ABL Cell Migration to hMSCs in Transwell

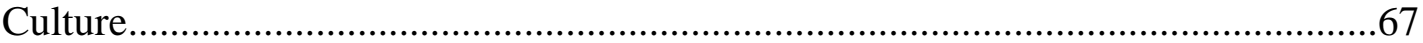

$1,25(\mathrm{OH})_{2} \mathrm{VD}_{3}$ Increased CXCL12 mRNA and Protein Expression in hMSCs in vitro and in Mouse Serum in vivo .........................................................................67

CXCL12 Increases the Number of BCR-ABL Cells in Suspension Culture ..............70

Vitamin D Plus ALL Reprograms the Bone Marrow Stroma..................................70

Vitamin D Deficiency Alters the Number of CXCL12 Expressing Cell Types in Bone Marrow and Bone .................................................................................... 70

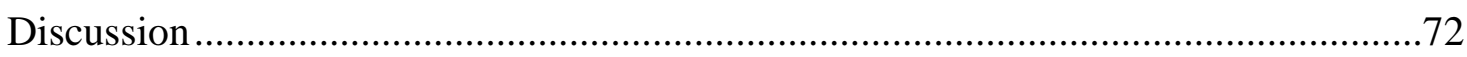

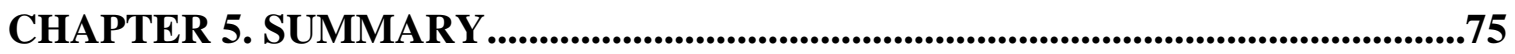

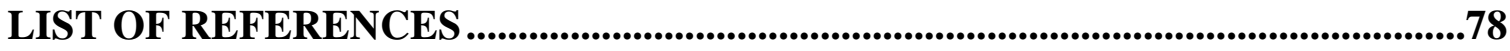

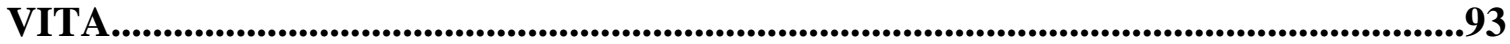




\section{LIST OF TABLES}

Table 3-1. Serum 25(OH)VD 3 levels in $\mathrm{VD}_{3}$-sufficient and -deficient mice (Representative date) ....................................................................29

Table 3-2. Repeat dexamethasone PK in drinking water. .......................................32

Table 3-3. Survival proportions of $\mathrm{VD}_{3}$-sufficient and -deficient mice treated with dexamethasone during survival study from BCR-ABL. ............................38

Table 4-1. Antibodies used to stain bone and bone marrow cells for flow cytometry...56

Table 4-2. Survival proportions of control and vitamin D-deficient mice...................60

Table 4-3. Serum 25-(OH) VD 3 values across groups (Representative data)..............61

Table 4-4. $\quad \mathrm{VD}_{3}$-sufficient (control), -deficient (VDD) male (M) mice complete blood count $(\mathrm{CBC})$..........................................................................62

Table 4-5. $\quad \mathrm{VD}_{3}$-sufficient and -deficient male mice serum chemistry.......................63 


\section{LIST OF FIGURES}

Figure 2-1. Schematic for specific aims.

Figure 3-1. Design to develop age-controlled vitamin D-sufficient and -deficient mice.

Figure 3-2. Experimental design for pharmacokinetics of DEX using discontinuous DEX regimen in drinking water.

Figure 3-3. Experimental design for pharmacokinetics of DEX post oral DEX gavage $(2 \mathrm{mg} / \mathrm{kg})$.

Figure 3-4. Experimental design for pharmacokinetics of dasatinib post oral dasatinib gavage $(10 \mathrm{mg} / \mathrm{kg})$.

Figure 3-5. Experimental design for survival of mice from BCR-ABL ALL placed on discontinuous DEX regimen in drinking water.

Figure 3-6. Cyp and transporter gene expression in duodenum of $\mathrm{VD}_{3}$ sufficient and deficient mice.

Figure 3-7. Cyp and transporter gene expression in liver and kidney of 12-week old hCYP3A4 mice.

Figure 3-8. Plasma dexamethasone concentrations in C57BL/6 VD 3 sufficient and deficient mice on a discontinuous dexamethasone regimen.

Figure 3-9. Plasma DEX pharmacokinetics in VD3-sufficient and -deficient mice after a single oral gavage of $2 \mathrm{mg} / \mathrm{kg}$ dexamethasone.

Figure 3-10. Induction of duodenal and hepatic Cyps in $\mathrm{VD}_{3}$-deficient mice after dexamethasone treatment.

Figure 3-11. Plasma dasatinib pharmacokinetics in $\mathrm{VD}_{3}$-sufficient and -deficient mice after a single oral gavage of $10 \mathrm{mg} / \mathrm{kg}$ dasatinib. .36

Figure 3-12. Xenogen images tracking disease burden in $\mathrm{VD}_{3}$-sufficient and deficient male mice injected with BCR-ABL ALL.

Figure 3-13. Kaplan-Meier curves for the effect of $\mathrm{VD}_{3}$ sufficiency vs. $\mathrm{VD}_{3}$ deficiency on survival of mice from BCR-ABL Arf-/- leukemia.

Figure 3-14. Serum chemistry of $\mathrm{VD}_{3}$-sufficient and -deficient mice.

Figure 3-15. Cartoon depicting dexamethasone and dasatinib metabolism and efflux transport in intestine and liver. 
Figure 3-16. Cartoon depicting hypothesis that dexamethasone-activated PXR can induce Cyp3a in $\mathrm{VD}_{3}$ deficient mice, but not in $\mathrm{VD}_{3}$-sufficient mice where $1,25(\mathrm{OH})_{2} \mathrm{D}_{3}$-activated VDR blocks DEX-PXR induction.

Figure 3-17. Differences in plasma and gut dexamethasone concentrations following oral discontinuous regimen leads to tissue specific activation of GR and PXR.

Figure 4-1. BCR-ABL ALL disease generation and monitoring in mice

Figure 4-2. BCR-ABL cell migration in vitro using transwell membrane .53

Figure 4-3. Experimental design - bone/bone marrow cells isolation and staining.........56

Figure 4-4. BCR-ABL luc+ ALL disease burden in male mice........................................58

Figure 4-5. BCR-ABL luc+ ALL disease burden in female mice....................................58

Figure 4-6. BCR-ABL ALL disease burden on day 17 in male control and VDD mice.

Figure 4-7. Kaplan Meier survival curve of male $\mathrm{VD}_{3}$ sufficient (control) and $\mathrm{VD}_{3}$ deficient mice.

Figure 4-8. Determination of mouse gender using Sly/Xlr and Zfy PCR.

Figure 4-9. Luminescence measure and leukemia score in control and VDD male mice.

Figure 4-10. Kaplan meier survival curves using male mice started on control (vitamin D sufficient) and VDD diet beginning at weaning. .65

Figure 4-11. Determination of VDR expression using PCR. .66

Figure 4-12. 1, 25(OH) $2 \mathrm{VD}_{3}$ increases BCR-ABL proliferation in vitro. .68

Figure 4-13. Increased migration of BCR-ABL leukemia cells to VD3 treated bone marrow stroma. .68

Figure 4-14. Vitamin D induces CXCL12 mRNA in bone marrow stroma in vitro. .69

Figure 4-15. Vitamin D induces CXCL12 protein expression in bone marrow stroma in vitro and in serum of mice in vivo.

Figure 4-16. CXCL12 increases proliferation of BCR-ABL ALL cells in suspension culture.

Figure 4-17. Effect of vitamin D and BCR-ABL leukemia on expression of type 1 and type $\mathrm{V}$ collagens in mouse bone marrow. 
Figure 4-18. Percentage of different CXCL12 expressing cells in bone marrow of $\mathrm{VD}_{3}$-sufficient mice versus $\mathrm{VD}_{3}$-deficient mice. ..........................................73

Figure 4-19. Percentage of different CXCL12 expressing cells in bone tissue of $\mathrm{VD}_{3}$ sufficient mice versus $\mathrm{VD}_{3}$-deficient mice...................................................73 


\section{LIST OF ABBREVIATIONS}

$\mathrm{ABC}$

ABCB 1

$\mathrm{ABCC} 4$

$\mathrm{ABCG} 2$

ALL

AML

BCRP

$\mathrm{BM}$

BMD

BMM

CAR

CARs

$\mathrm{Cl}$

CLL

CML

CYP

DBP

DEX

GR

HAT

HBSS

hMSCs

HPMC

HSCs

IL-2

MRP4

MSCs

NHL

NR

NR1I1

NR1I2

NR1I3

PGP

$\mathrm{Ph}+$

PK

PTH

PXR

RXR

SDF-1

SRC-1

SRC-2

SRC-3

V1

ATP-binding cassette

ABC subfamily $B$ member 1

ABC subfamily $C$ member 4

ABC subfamily $G$ member 2

Acute lymphoblastic leukemia

Acute myeloid leukemia

Breast cancer resistant protein

Bone marrow

Bone mineral density

Bone marrow microenvironment

Constitutive androstane receptor

CXCL12 abundant reticular cells

Clearance

Chronic lymphoid leukemia

Chronic myeloid leukemia

Cytochrome P450

Vitamin D binding protein

Dexamethasone

Glucocorticoid receptor

Histone acetylase activity

Hank's balanced salt solution

Human marrow-derived stromal cells

Hydroxypropyl methyl cellulose

Hematopoietic stem cells

Interleukin-2

Multidrug resistance associated protein 4

Mesenchymal stem cells

Non-Hodgkin's lymphoma

Nuclear hormone receptor

NR subfamily 1 , group 1 , member 1

NR subfamily 1, group 1, member 2

NR subfamily 1, group 1, member 3

P-glycoprotein

Philadelphia chromosome

Pharmacokinetics

Parathyroid hormone

Pregnane X Receptor

Retinoid X Receptor

Stromal cell derived factor 1

Steroid receptor activator 1

Steroid receptor activator 2

Steroid receptor activator 3

Volume of distribution 


$\begin{array}{ll}\mathrm{VD}_{3} & \text { Vitamin D } \\ \text { VDD } & \text { Vitamin D deficiency } \\ \text { VDR } & \text { Vitamin D Receptor } \\ \text { VDREs } & \text { Vitamin D response elements } \\ 1,25-(\mathrm{OH})_{2} \mathrm{VD}_{3} & \text { 1, 25-dihydroxy vitamin D } \\ 25-(\mathrm{OH})_{2} \mathrm{VD}_{3} & \text { 25-hydroxy vitamin D }\end{array}$




\section{CHAPTER 1. INTRODUCTION}

Cytochrome P450s (CYP) play significant roles in detoxification of many exogenous chemicals, drugs and toxins. These enzymes have broad substrate specificity and metabolize various endogenous and natural products as well. CYP's are not only involved in drug disposition but also contribute significantly to first-pass metabolism of many drugs. Various drugs and endogenous compounds like vitamin D can transcriptionally induce CYP3A. Depending on the dose and route of inducing agents, the extent of intestinal and hepatic induction may differ. Moreover, intestinal CYP3A expression is sensitive to seasonal changes correlating with seasonal vitamin D levels (Thirumaran et al., 2012). Consequently the induction of CYP's can lead to decreased oral bioavailability of many drugs that are metabolized by CYP3A (Kaminsky \& Fasco, 1991). The following chapter highlights the role of vitamin $D$ in transcriptional regulation of cytochrome P450s through various receptors and associated drug interactions.

\section{Overview of Vitamin D}

\section{Vitamin D Bioactivation}

Vitamin D (VD3) is a seco-steroid hormone which can be synthesized from 7dehydrocholesterol when skin is exposed to ultraviolet radiation. $\mathrm{VD}_{3}$ is hydroxylated in the liver into 25-hydroxy vitamin $\mathrm{D}\left(25-(\mathrm{OH}) \mathrm{VD}_{3}\right)$ by two different vitamin $\mathrm{D}-25-$ hydroxylases (R. Kumar, 1984): CYP27A1, CYP2R1 which are present in hepatic microsomes and mitochondria. $25-(\mathrm{OH}) \mathrm{VD}_{3}$ is the major circulating form of $\mathrm{VD}_{3}$ (Henry $\&$ Norman, 1984) and it tightly binds to vitamin D binding protein (DBP) in the plasma. It has a long plasma half-life ( 14 days) and is generally used as a measure of vitamin D status (Holick, 2007). 25-(OH) $\mathrm{VD}_{3}$ is further hydroxylated into a biologically active form, 1,25-dihydroxy vitamin D $\left(1,25-(\mathrm{OH})_{2} \mathrm{VD}_{3}\right)$ mainly by CYP27B1 in kidney (Hewison et al., 2007). However, CYP27B1 is also expressed in extra-renal tissues such as intestinal epithelial cells, keratinocytes, dendritic cells, cancer cells etc. where 1,25$(\mathrm{OH})_{2} \mathrm{VD}_{3}$ is locally formed (Feldman, Krishnan, Swami, Giovannucci, \& Feldman, 2014; Gao, Liao, Han, Thummel, \& Mao, 2018). Well known intestinal effects mediated by $1,25-(\mathrm{OH})_{2} \mathrm{VD}_{3}$ could be a result of local bioactivation of $\mathrm{VD}_{3}$ via. CYP27B1 (Gawlik et al., 2015). CYP27B1 not only increases $1,25-(\mathrm{OH})_{2} \mathrm{VD}_{3}$ levels but also decreases its metabolite, $24,25-(\mathrm{OH})_{2} \mathrm{VD}_{3}$ levels by converting it into $1,24,25-(\mathrm{OH})_{3} \mathrm{VD}_{3}$ (Inouye \& Sakaki, 2001).

\section{Vitamin D Inactivation}

Both 25-(OH) VD 3 and 1,25- $(\mathrm{OH})_{2} \mathrm{VD}_{3}$ are converted into polar metabolites via two specific pathways, the first step being the hydroxylation of 1,25- $(\mathrm{OH})_{2} \mathrm{VD}_{3}$ to 1,24R,25-(OH $)_{3} \mathrm{VD}_{3}$ and further oxidation leading to a terminal product, calcitroic acid 
(Endres \& DeLuca, 2001). CYP24A1 (24-hydroxylase) is the enzyme that catalyzes the hydroxylation at C-24 and C-23 positions of vitamin D metabolites, which is physiologically the main route of vitamin D degradation (Christakos, Dhawan, Verstuyf, Verlinden, \& Carmeliet, 2016). CYP24A1 is the mitochondrial P450 mainly expressed in kidney and other vitamin D target tissues such as intestine (R. Kumar, 1984) and bone (Turner et al., 1980). The other P450 enzyme which can also metabolize $1,25-(\mathrm{OH})_{2} \mathrm{VD}_{3}$ was later reported to be CYP3A4 contributing to form 23R- and 24S-hydroxy metabolites, which are stereo chemically opposite to the metabolites produced by CYP24A1 (Xu et al., 2006). Hydroxylation at C-23 is preferred by CYP3A4 and represents a dominant pathway of $1,25-(\mathrm{OH})_{2} \mathrm{VD}_{3}$ metabolism in liver and intestine.

\section{Vitamin D Regulation}

Target cells of vitamin D have the ability to regulate its metabolism at the cellular level indicating the importance of cellular concentration of $1,25(\mathrm{OH})_{2} \mathrm{VD}_{3}$ by which tissues regulate their responsiveness to vitamin D (G. Jones, Strugnell, \& DeLuca, 1998). Moreover, vitamin D bio-activation and degradation is tightly regulated to maintain appropriate levels of active vitamin D and its physiological effects (G. Jones et al., 1998). For example, 1,25- $(\mathrm{OH})_{2} \mathrm{VD}_{3}$ can itself stimulate CYP24A1 to increase its metabolism whereas low calcium and parathyroid hormone $(\mathrm{PTH})$ levels can inhibit CYP24A1 to maintain the active vitamin D levels (Christakos et al., 2016). Similar to this negative feedback mechanism in kidney, CYP3A4 is also prone to negative feedback mechanism by constitutive $1,25-(\mathrm{OH})_{2} \mathrm{VD}_{3}$ in intestine (Xu et al., 2006). It has been reported that 1,25- $(\mathrm{OH})_{2} \mathrm{VD}_{3}$ can regulate intestinal Cyp3a in mice (Makishima et al., 2002), suggesting that induction of CYP3A4 by $1,25(\mathrm{OH})_{2} \mathrm{VD}_{3}$ can in turn result in enhanced metabolism of $1,25(\mathrm{OH})_{2} \mathrm{VD}_{3}$ itself, thereby mitigating the extent of its cellular effects.

\section{Vitamin D and Vitamin D Receptor}

Different forms of vitamin D can bind to DBP which transports vitamin D between tissues such as liver, kidney, skin and to various target tissues including intestine and bone. When the biologically active form of vitamin $\mathrm{D}, 1,25-(\mathrm{OH})_{2} \mathrm{VD}_{3}$ enters plasma, it is taken up by target tissues expressing a cytosolic receptor known as the vitamin $\mathrm{D}$ receptor (VDR). $1,25(\mathrm{OH})_{2} \mathrm{VD}_{3}$ binds to VDR with high affinity, generally in the range of $10^{-10} \mathrm{M}$ (DeLuca \& Schnoes, 1976).

VDR (NR1I2) belongs to nuclear receptor (NR) superfamily and acts as a transcription factor when ligand activated. When $1,25-(\mathrm{OH})_{2} \mathrm{VD}_{3}$ binds to VDR, the complex forms a heterodimer with the retinoid $\mathrm{X}$ receptor (RXR) and binds to vitamin $\mathrm{D}$ response elements (VDREs) on target DNA sequence with high affinity. Following this, recruitment of transcriptional coactivators, such as steroid receptor activator $1,2,3$ (SRC-1, SRC-2, SRC-3) that has histone acetylase activity (HAT), is required to either activate or repress transcription of target genes. These coactivators bind to the AF2 
domain of the liganded VDR and act as master regulators of $1,25-(\mathrm{OH})_{2} \mathrm{VD}_{3}$ action (Christakos et al., 2016; Haussler et al., 2013).

VDR is expressed in many tissues in vivo but at relatively low levels compared to its main target tissues such as bone, kidney and intestine which have high VDR expression (Dame, Pierce, \& DeLuca, 1985). It is also expressed in immune cells, specially T-cells where it modulates interleukin -2 (IL-2) levels (Dame et al., 1985).

\section{Overview of Vitamin D}

\section{CYP3A4/Cyp3a Gene}

CYP enzymes are a superfamily of hemeproteins that metabolize a wide range of xenobiotics including therapeutic drugs, toxins, carcinogens as well as endogenous molecules (Nebert \& Russell, 2002). Three P450 families CYP1, CYP2, CYP3 are currently known to be involved in drug metabolism (Furge \& Guengerich, 2006). In humans, the CYP3A subfamily is critical for drug clearance and is reported to metabolize at least $50 \%$ of the currently prescribed drugs (Y. T. Liu, Hao, Liu, Wang, \& Xie, 2007) (Guengerich, 2008). The CYP enzymes not only play a significant role in therapeutic drug metabolism and disposition, but they are also responsible for many adverse drug reactions. Clinical data suggests sex and age dependent tissue distribution of CYP enzymes and huge interindividual variability.

\section{Localization of CYP3A4/Cyp3a}

Most CYP enzymes are highly expressed in tissues involved in absorption, metabolism and excretion such as liver, small intestine and kidney. Almost $70 \%$ of human P450's is composed of CYP1A2, 2A6, 2B6, 2C, 2D6, 2E1 and 3A isoforms. CYP3A4, CYP3A5, CYP3A7 and CYP3A43 are the four isoforms of the CYP3A family among which CYP3A4 and CYP3A5 account for almost $30 \%$ and $20 \%$ of total P450 respectively. In humans, CYP3A4 is highly expressed in mucosal epithelium of duodenum and jejunum (Paine et al., 1997) (Watkins et al., 1985). It is also predominantly expressed in normal liver (Achour, Barber, \& Rostami-Hodjegan, 2014). Hepatic distribution of CYP's is relatively homogenous compared to small intestine where both the content and activity of CYPs is higher in the proximal than the distal regions.

In mice, the Cyp3a family genes were primarily detected in liver and intestine. Liver expresses high levels of at least six out of eight Cyp3a isoforms Cyp3a11, 3a16, 3a25, 3a41a/b, 3a44 and 3a59 in mice. Whereas, Cyp3a11 is the most highly expressed Cyp3a in intestine compared to other Cyp3a's, expression of Cyp3a13 is higher in intestine than liver, and Cyp3a59 is higher in testis than liver. Similar to humans, mouse 
hepatic Cyp3a exhibit sexual dimorphism with females expressing predominantly Cyp3a16, 41a/b, 3a44 and 3a59.(Renaud, Cui, Khan, \& Klaassen, 2011).

\section{Regulation of CYP3A}

Nuclear hormone receptors (NR) such as the pregnane $\mathrm{X}$ receptor (PXR) (or NR subfamily 1, group 1, member 2, NR1I2), constitutive androstane receptor (CAR) (or NR subfamily 1, group 1, member 3, NR1I3) and VDR (or NR subfamily 1, group 1, member 1, NR1I1) are known to play a significant role in the regulation of CYP3A genes. PXR and CAR are regarded as xenosensors regulating detoxification and elimination of xenobiotics or toxic endogenous substances from the body (Mackowiak \& Wang, 2016). Interestingly the other NR1 family member VDR also regulates numerous drugmetabolizing enzymes (Pavek \& Dvorak, 2008). Unlike VDR, PXR is highly expressed in liver, but also in intestine and to a lesser extent in kidney (Pavek, 2016). Studies have reported that PXR is involved in the regulation of other CYPs such as CYP2B6, 2C9, 2C29, 3A5, 3A7 and transporters such as UGT1A1, P-glycoprotein (Pavek \& Dvorak, 2008).

As suggested by recent studies PXR and CAR have the potential to cross-regulate CYP3A expression by different mechanisms either by sharing some ligands which could result in dual activation or binding of PXR and CAR to common target DNA response elements. But it has been reported that PXR is a dominant regulator of CYP3A expression compared to CAR (Goodwin, Hodgson, \& Liddle, 1999).

Endogenous compounds and xenobiotics that are substrates to these NR's regulate CYP3A expression and contribute to huge inter individual variability in humans. Numerous other factors including genetic and environmental could contribute to the inter-individual variability of intestinal CYP3A4 but cannot explain it completely. Many studies reported clinically observed variability in intestinal CYP3A expression (Lown et al., 1994; Paine et al., 2005; Xu et al., 2006). Pharmacokinetics and pharmacodynamics of many drugs that are CYP3A4 substrates can be influenced by this variability (Gorski et al., 1998; Thummel et al., 1996) which can influence the extent of drug absorption, metabolism and elimination thereby altering the therapeutic efficacy or toxic response of the drug.

\section{Substrates and Inducers of CYP3A}

\section{Substrates}

The CYP3A family of enzymes are known to metabolize a variety of clinical drugs including macrolide antibiotics such as rifampin and naturally occurring and synthetic glucocorticoids such as dexamethasone (DEX). However, xenobiotic exposure can result in enhanced or repressed transcription of both intestinal and hepatic CYP3A. 
Many of CYP3A substrates can also induce CYP3A expression. For example, DEX is both substrate and inducer of CYP3A4.

\section{Inducers}

Steroid hormones including glucocorticoid agonists and antagonists, macrolide antibiotics, imidazole antifungals, phenobarbital, polychlorinated biphenyls and organic pesticides are known to induce CYP3A transcription (Gonzalez, Liu, \& Yano, 1993). Drugs such rifampin, phenobarbital, glucocorticoids can induce mouse Cyp3a11, Cyp3a13 and human CYP3A4 in vivo and in cultured hepatocytes (Schuetz, Wrighton, Barwick, \& Guzelian, 1984; Watkins et al., 1985; Wrighton et al., 1985; Yanagimoto, Itoh, Sawada, \& Kamataki, 1997). The CYP2B family genes are also normally induced by many of these same drugs (Honkakoski \& Negishi, 1998; Strom et al., 1996). These interactions lead to clinically important drug-drug interactions when these inducer drugs are co-administered with CYP substrates (such as cyclosporin A, midazolam etc.).

\section{Species Differences}

Marked species differences exist regarding CYP3A induction. For example, in humans, rifampin causes a 10-fold induction of CYP3A (Strolin Benedetti \& Dostert, 1994), whereas only a 2-fold induction in mice (Tredger, Smith, Powell-Jackson, Davis, \& Williams, 1981). Pregnenolone-16a-carbonitrile (PCN) is a strong inducer of rat CYP3A but not human.

\section{Role of PXR, GR in CYP3A Induction}

PXR is the main receptor involved in the steroid and drug induction of CYP3A (Kliewer et al., 1998). High concentrations of DEX and phenobarbital ligand activate both mouse and human PXRs to induce CYP3A. There is no role for the glucocorticoid receptor (GR) in CYP3A induction. Glucocorticoids and anti-glucocorticoids follow the non-classic glucocorticoid induction pathway and induce CYP3A genes (Schuetz et al., 2000). Although glucocorticoids are both substrates and inducers of CYP3A enzymes, they have different requirements for the glucocorticoid receptor to induce CYP3A and CYP2B genes (Schuetz et al., 2000). In normal condition, they can bind to GR and induce genes regulated by GR. However, when glucocorticoid levels are extremely high (stressful conditions) or when high doses are given, they can produce maximum CYP3A induction through activation of PXR (Yusim, Franklin, Brooke, Ajilore, \& Sapolsky, 2000). The dose required to activate PXR is usually at least 100-fold higher (Reid, 1997) than that required to activate GR. Physiologically, this feedback mechanism of PXR mediated CYP3A induction is to regulate the levels of corticosteroids and maintain steroid homeostasis to prevent any tissue damage during stressful conditions $(\mathrm{H}$. Zhang et al., 1999) (Collier, Wu, \& Pruett, 1998). The significant difference in the ligand binding domain (LBD) of PXR across species is responsible for the species differences in 
induction of CYP3A - e.g., rifampin potently activates the LBD of human PXR, but is a weak activator of rat and mouse PXR, while PCN activates rodent PXRs LBD but not human PXR LBD.

(Schuetz et al., 1984) reported that GR might be involved in the synergistic induction of CYP3A by PCN and DEX. This synergistic induction could be a result of either protein-protein interaction between GR and PXR or inducers binding to glucocorticoid response elements (GRE) present upstream of PXR/RXR binding site on CYP3A gene. Moreover, recently it was reported that DEX treatment increased PXR and RXR mRNA accumulation in human hepatocytes likely through GR activation, which could also result in increased CYP3A expression (H. Zhang et al., 1999). However, induction of PXR and RXR mRNA requires their levels in hepatocytes to be rate limiting.

\section{Dietary Interactions with CYP3A}

Significant clinical interactions due to CYP3A induction or repression of activity by not only drugs but also dietary and natural compounds have been reported. For example, St. John's wort and grapefruit juice have been reported to alter CYP3A enzymes (Bailey, Malcolm, Arnold, \& Spence, 1998; Bhardwaj et al., 2002; Johnson, Won, Kock, \& Paine, 2017; Peltoniemi et al., 2012). Mechanistically, St. John's wort contains hyperforin one of the most potent ligand activators of PXR. Grapefruit juice contains 6,7-dihydrobergamottin that increases proteasome mediated degradation of intestinal CYP3A4 protein while having no effect on its mRNA. At least 20 drugs especially those with extremely low oral bioavailability due to pre-systemic metabolism were assessed to undergo interactions with grapefruit juice. Moreover, these interactions differ from patient to patient based on type and amount of grapefruit juice and individual susceptibility (Bailey et al., 1998). This indicates that dietary ingredients can also influence the level of CYP3A expression and therefore explain partly the huge interindividual variability in humans.

Similarly, vitamin D is also an important transcriptional regulator of CYP3A expression, and CYP3A is sensitive to changes in vitamin D levels. Consequently, vitamin D levels can also contribute to the CYP3A expression variability depending on the individual vitamin D status. These vitamin D-CYP3A interactions could be of significant importance specially when the co-administered drugs have a narrow therapeutic index or major toxicity.

\section{Drug Transporters}

The ATP-binding cassette (ABC) family of transporters are well known efflux transporters that transport wide range of drugs, exogenous chemicals and endogenous substances. P-glycoprotein (PGP) is a member of ABC subfamily B member 1 (ABCB1) which is present in multiple tissues but highly expressed in intestine (epithelial cells), liver (hepatocytes) brain, kidney (Allen, Brinkhuis, Wijnholds, \& Schinkel, 1999). It 
plays a significant role in protecting our body from toxins. Similar to CYP3A family, transporters also have a protective function by reducing the absorption of toxic chemicals, and hence there is a lot of substrate overlap between drug metabolizing enzymes and transporters (Borst \& Schinkel, 2013), (Cascorbi, 2011). Dexamethasone, dasatinib, cyclosporin etc. are substrates of both CYP3A and PGP (Schinkel, Wagenaar, van Deemter, Mol, \& Borst, 1995) (Schinkel et al., 1995).

Breast cancer resistant protein (BCRP) or ABC subfamily G2 member (ABCG2) is an efflux transporter mainly expressed in placenta, small intestine, liver, kidney, blood brain barrier and breast (Stacy, Jansson, \& Richardson, 2013). BCRP transports many endogenous substances and a diverse groups of exogenous chemicals such as tyrosine kinase inhibitors, anthracene drugs, statins, and proton pump inhibitors, etc. (Mao \& Unadkat, 2015; Meyer zu Schwabedissen \& Kroemer, 2011).

Multidrug resistance associated protein (MRP4) or ABC subfamily $\mathrm{C}$ member 4 $(\mathrm{ABCC} 4)$ is another well-known transporter that transports many endogenous and exogenous substances. It plays a significant role in renal excretion of its substrates (Russel, Koenderink, \& Masereeuw, 2008).

Drug metabolizing enzymes and transporters are major routes of detoxification for the drugs in the body and also share substrates and inhibitors. Therefore, they contribute significantly to the drug-drug interactions and evaluating their expression in vitamin $\mathrm{D}$ deficiency condition could provide insights into mechanisms involved in vitamin drug interactions.

\section{Vitamin D Deficiency}

Vitamin D deficiency (VDD) is very common in a significant number of people worldwide and is considered endemic in many parts of the world with deficiency rates ranging from 42 to $61 \%$ in pediatric and adolescent population in the United States (J. Kumar, Muntner, Kaskel, Hailpern, \& Melamed, 2009). Although lack of exposure to sunlight and low vitamin D intake are considered to cause vitamin D deficiency, clinically impaired intestinal vitamin D absorption or treatment with drugs enhancing vitamin D metabolism can also result in VDD (Glorieux \& Pettifor, 2014; Prentice, 2013; Schafer et al., 2015). Vitamin D deficiency could lead to many disorders such as type II Rickets, increased bone fracture, etc.

Therefore, vitamin D supplementation is generally given to maintain vitamin D levels and to increase the bone mineral density in patients. Although many clinical trials investigated the effect of vitamin D supplementation with or without calcium to reduce fracture incidence, there is no consensus on the benefit of supplementing vitamin $D$ in these patients (Bischoff-Ferrari et al., 2012; Lips, Gielen, \& van Schoor, 2014). Furthermore, there is a concern that vitamin D supplementation could cause vitamin drug interactions with CYP3A4 substrates. 


\section{Vitamin D - CYP3A4 Clinical Interactions}

Vitamin D (VD3) deficiency leads to bone loss, decreased bone mineral density, rickets, osteoporosis, hyperparathyroidism and increased risk of fracture (Greer, 2009). Glucocorticoids such as DEX and prednisone are widely used clinically as part of leukemia chemotherapy. Long term exposure to glucocorticoids is known to produce adverse skeletal effects due to early rapid bone resorption and a late impaired bone formation (Mazziotti, Giustina, Canalis, \& Bilezikian, 2007). This adverse effect is also attributed to increased $\mathrm{VD}_{3}$ metabolism which can be detrimental to the $\mathrm{VD}_{3}$ deficient leukemia patients.

\section{Vitamin D - Intestinal CYP3A4}

Since intestinal CYP3A4 mediated metabolism contributes to decreased oral bioavailability of its substrates, co-administration of VD 3 (a CYP3A4 inducer) with a CYP3A4 substrate might cause incomplete oral bioavailability of that drug substrate. On the other hand, vitamin D deficiency could increase the oral bioavailability of CYP3A4 substrates. A study by Schwartz et al., reported increased clearance of a CYP3A4 substrate, atorvastatin in vitamin D deficient humans supplemented with $\mathrm{VD}_{3}$ (Schwartz, 2009). Another study reported seasonal variation in serum levels of CYP3A4 substrates, tacrolimus and sirolimus that mirrored the seasonal variation in serum levels of $\mathrm{VD}_{3}$ (Lindh, 2011). Previously our lab has reported the seasonal variation of in vivo intestinal CYP3A4 expression, correlating with seasonal VD3 levels (Thirumaran et al., 2012).

Hence, serum VD3 levels, which is associated with intestinal CYP3A4 activity, has the potential to alter oral drug absorption and clearance. At St. Jude Children's Research Hospital, CYP3A4 substrates such as DEX and dasatinib are administered orally as part of chemotherapy to ALL patients (Christopher et al., 2008; Gentile, 1996).

Dexamethasone (DEX) is a synthetic analog of cortisol used during the induction, delayed intensification, and maintenance phases of ALL chemotherapy, most pediatric patients receive it for about half of their 2-3 years of treatment period (Margolin JF, 2006). Because DEX is both a substrate and inducer of CYP3A, understanding the effect of $\mathrm{VD}_{3}$ deficiency on DEX systemic exposure and evaluating any vitamin drug interactions is necessary.

Dasatinib is a tyrosine kinase inhibitor, specifically administered to acute lymphoblastic leukemia patients with the BCR-ABL-translocation that generates the BCR-ABL+ oncogene that has elevated ABL kinase activity. Dasatinib can also inhibit SRC kinase which is involved in growth and progression of many cancers. Dasatinib is specifically approved to be used in imatinib resistant chronic myeloid leukemia and acute lymphoblastic leukemia. The oral bioavailability of dasatinib is very low ranging from $14 \%$ in mouse to $34 \%$ in dogs. Dasatinib undergoes high first-pass metabolism by CYP3A, both in the intestine and liver (Christopher et al., 2008). Any changes in 
intestinal CYP3A due to vitamin D status might decrease or increase the pre-systemic metabolism of dasatinib in the gut, thereby influencing its pharmacokinetics.

Because both these drugs are administered orally, we evaluated whether changes in pharmacokinetics and clearance of dexamethasone and dasatinib occurred due to $\mathrm{VD}_{3}$ deficiency.

In summary, small intestine is the first site of exposure to orally administered drugs where abundant drug metabolizing CYP3A enzymes and transporter are expressed. They are responsible for increasing or decreasing the oral bioavailability of many drugs. Because CYP3A4 has broad substrate specificity, many drugs have the potential to interact with CYP3A4 and cause drug-drug interactions. Most of these interactions have been studied and reported. In spite of many reports suggesting vitamin D deficiency in normal population as well as patients with various cancers, very little is known about vitamin D's interaction with CYP3A4 substrates. Because pediatric acute lymphoblastic patients are orally administered DEX and dasatinib, substrates of CYP3A4, it was important to determine whether supplementation of vitamin $\mathrm{D}$ to $\mathrm{VD}_{3}$ insufficient/deficient patients might cause a drug interaction with either substrate or compromise anti-leukemic efficacy. 


\section{CHAPTER 2. VITAMIN D AND LEUKEMIA}

\section{Vitamin D in Health}

Vitamin D regulates many different genes, most of which play an important role in physiological calcium homeostasis or bone formation. These genes include osteocalcin, osteopontin, parathyroid hormone (PTH) (Berridge, 2015). In intestine, $1,25(\mathrm{OH})_{2} \mathrm{VD}_{3}$ is known to regulate many processes such as intestinal calcium and phosphorus transport, nuclear events such as chromatin template activity, DNAdependent RNA polymerase II activity and $\mathrm{Ca}^{+2}$ dependent ATP and alkaline phosphatase activities (Carlberg \& Campbell, 2013). Although maintaining calcium and phosphate homeostasis is an important function of $1,25-(\mathrm{OH})_{2} \mathrm{VD}_{3}$, many studies have demonstrated its role in other cellular processes such as proliferation, differentiation, immune modulation in both normal and malignant cells (Berridge, 2015; Bikle, 2012).

\section{Vitamin D in Disease}

Vitamin D promotes enterocyte differentiation and intestinal calcium and phosphorus absorption which helps in bone mineralization. Therefore, vitamin D deficiency can significantly affect the bone mineral density both by reduced absorption and enhanced resorption of calcium resulting in several disorders such as rickets and osteomalacia. CYP27B1 converts $25(\mathrm{OH}) \mathrm{VD}_{3}$ to $1,25(\mathrm{OH}) \mathrm{VD} 3$, and mutations in this gene resulting in inactive or deleted CYP27B1 cause vitamin D deficiency rickets type I (VDDRI) irrespective of normal vitamin D intake. Hereditary vitamin D resistant rickets (HVDDR or VDDRII) is another form of rickets where there is organ-resistance to vitamin D due to heterogenous loss of function mutations in VDR (Sahay \& Sahay, 2012). Because calcium and bone homeostasis are highly interlinked, studies in both mice or humans lacking VDR or CYP27B1 has shown phenotype of rickets and osteomalacia. In spite of differences in role of vitamin $\mathrm{D}$ between human and mouse models, animal models could still help unravel complex mechanistic pathways involving similar genes that could affect humans (Christakos et al., 2016).

Epidemiological studies suggested a role of vitamin D not only in the skeletal system but also in cancer, cardiovascular disease, kidney disease, psoriasis etc. (Sahay \& Sahay, 2012). Many studies have reported that vitamin D deficiency is associated with cardiovascular pathology. In humans, low vitamin D levels are associated with arterial hypertension, diabetes mellitus and dyslipidemia (Podzolkov, Pokrovskaya, \& Panasenko, 2018). Because of vitamin D's role in various cellular processes such proliferation, apoptosis, ageing of cells, it has been associated with various age-related diseases such as Alzheimer's, Parkinson's, multiple sclerosis diseases (Berridge, 2017). Although laboratory studies report extra-skeletal beneficial effects of supplementing vitamin $\mathrm{D}$, it is important to obtain definite clinical data regarding the use of vitamin $\mathrm{D}$ and its analogues for treatment or prevention of disease processes. 


\section{Vitamin D in Cancer}

Many cancer cells not only express VDR through which vitamin D exhibits its effects, but also express CYP27B1 or CYP24A1, the enzymes involved in vitamin D metabolism (Bikle, 2016). The expression of these enzymes can regulate the local production of active $\mathrm{VD}_{3}$, which can have significant implications in cancer progression (Hobaus, Thiem, Hummel, \& Kallay, 2013; Swami et al., 2012). Several reports have suggested VDR expression in tumor cells to be a prerequisite for antineoplastic effects of $1,25-(\mathrm{OH})_{2} \mathrm{VD}_{3}$ such as differentiation of HL60 leukemia cells into macrophages requires VDR expression (Abe et al., 1981).

\section{Vitamin D Deficiency in Cancer}

It has been reported that at least $72 \%$ of pediatric and young adults with cancer suffer vitamin D deficiency at diagnosis and this hypovitaminosis persists throughput the survivorship (Choudhary, Chou, Heller, \& Sklar, 2013; Helou et al., 2014; Wallace et al., 2015). With significant proportions of cancer patients being vitamin D deficient, it became important to understand its association with the survival outcome. Several reports from pre-clinical as well as clinical studies show that vitamin D deficiency correlated with poor survival outcome. Vitamin D deficient mice inoculated with tumor cells such as colon or breast cancer had increase in tumor growth (Ooi et al., 2010; Ray et al., 2012; Tangpricha et al., 2005). On the other hand, repression of tumor formation was observed in rodents with sufficient vitamin D and calcium levels (Newmark et al., 2009).

In mouse xenograft models of prostrate and breast cancer, it has been reported that vitamin D supplementation can inhibit tumor growth (Swami et al., 2012). In a preclinical study involving mice with pancreatitis, administration of calcipotriol, an analogue of vitamin D resulted in decreased fibrosis and inflammation. Induction of extensive stromal remodeling with reduced tumor-supportive signaling led to enhanced efficacy of co-administered chemotherapeutic agent and increased survival of the mice (N. Ding et al., 2013). In spite of vitamin D's (supplementation) ability to reduce tumor growth and metastasis, its adverse effects such as hypercalcemia hinder its clinical applicability.

\section{Vitamin D Deficiency in Leukemia}

One of the major factors leading to leukemia is perturbations in white blood cell ( $\mathrm{T}$ or $\mathrm{B}$ cell) differentiation in the bone marrow (BM) due to a combination of genetic and environmental factors. Based on the lineage and persistence of the disease, leukemia is classified into four major types, acute lymphoblastic leukemia (ALL), chronic lymphoblastic leukemia (CLL), acute myeloid leukemia (AML), chronic myeloid leukemia (CML). Considering the prevalence of $\mathrm{VD}_{3}$ deficiency in leukemia patients, numerous researchers conducted studies to perceive the association of $\mathrm{VD}_{3}$ and clinical outcome in different types of leukemia. 
AML is characterized by immature hematopoietic myeloid precursors that have lost their ability to differentiate and proliferate normally (Tenen, 2003). Vitamin D deficiency is highly prevalent in AML patients with at least $80 \%$ of the patients at diagnosis have significantly low serum vitamin D levels (Seyedalipour et al., 2017) and has been associated with poor survival outcome whereas high vitamin D levels with better clinical outcome (Seyedalipour et al., 2017). AML cells, although expressing low constitutive levels of VDR, induced VDR expression at a post transcriptional level following treatment with 1,25-(OH) $2 \mathrm{VD}_{3}$ (Gocek, Baurska, Marchwicka, \& Marcinkowska, 2012). In vitro studies using a variety of AML cell lines such as HL-60, AML-193, NB-4, U937 etc. indicated 1,25-(OH $)_{2} \mathrm{VD}_{3}$ treatment induced differentiation of these cells into monocytes (Kim et al., 2012). In vivo studies using animal models with AML and treated with vitamin D analogues suggested significantly better survival of mice compared to the no treatment group (J. Y. Zhou et al., 1990). However, due to the limitations and inconsistencies between the studies, it is challenging to translate vitamin $\mathrm{D}$ or its analogues usage in the clinical setting (Cao et al., 2017).

Similar to AML, CLL patients also suffer with vitamin D insufficiency and that has been associated with poor overall survival outcome. Although higher $25-(\mathrm{OH}) \mathrm{VD}_{3}$ levels show protective effect against development of CLL, no significant correlation between vitamin D levels and overall risk for lymphoid cancer development has been shown (Luczynska et al., 2013). In a study with 983 non-Hodgkin's lymphoma (NHL) patients comprised of subtypes including diffuse large B-cell lymphoma and T-cell lymphoma, $44 \%$ of patients were vitamin D deficient at the time of diagnosis (Drake et al., 2010). These insufficient serum vitamin D levels were associated with shorter event free and overall survival specifically in these subtypes. No correlations were observed in other NHL subtypes (Bittenbring et al., 2014).

These studies indicate the importance of measuring vitamin D levels at diagnosis, throughout the treatment and evaluating if vitamin D has any effect on the clinical outcome.

\section{Vitamin D Deficiency in ALL}

Approximately $60 \%$ of ALL patients are vitamin D deficient (Young et al., 2018) and are highly prone to the adverse bone effects associated with chemotherapy. Persistent decline in bone mineral density is observed from diagnosis until off-therapy for as long as 10-years following diagnosis in ALL patients (Demirsoy et al., 2017; Kuhl et al., 2012). Reasons include vitamin D deficiency at diagnosis, leukemic cell infiltration, irradiation, toxicities associated with chemotherapies such as corticosteroids and methotrexate which are known to intensify bone resorption and decrease osteoblast function. Supportive care measures such as antiepileptic agents also negatively impact vitamin D levels by interacting with CYP3A4, an enzyme involved in vitamin D metabolism/inactivation (Heaney, 2005; J. Y. Lee, So, \& Thackray, 2013). 
Unlike other leukemia malignancies, association of vitamin D levels and survival outcome has not been studied in ALL. One of the reasons for this could be that B-ALL cells do not express VDR, which is considered to be a prerequisite for vitamin D's antineoplastic activity. There are at least two clinical trials ((Dana Farber and Children's Hospital of LA (ClinicalTrials.gov Identifier number: NCT01574274 and NCT01317940, respectively) administering vitamin $\mathrm{D}$ to insufficient and deficient pediatric acute lymphoblastic leukemia patients to restore VD3 sufficiency and determine if there is improved bone mineral density in the context of changes in body composition and body fat. In another study children and young adult ALL patients were supplied with vitamin D to maintain their bone mineral density and it was shown to be safe and effective in (Young et al., 2018). Nevertheless, long term health outcomes commonly observed in ALL patients such as osteonecrosis, fracture rate, and overall survival outcome have not been reported.

Since a significant proportion of ALL patients are $\mathrm{VD}_{3}$ insufficient/deficient, it is critical to evaluate the role of vitamin $\mathrm{D}$ status in the progression of such disorders. Therefore, we aimed to investigate the effect of $\mathrm{VD}_{3}$ on survival from BCR-ABL ALL in a mouse model. BCR-ABL is a fusion gene, also called Philadelphia chromosome $(\mathrm{Ph}+)$, that arises from the translocation between chromosomes 9 and 22 in at least 3-5\% of pediatric ALL cases and 25\% of adult ALL cases (Williams \& Sherr, 2008). The resulting $\mathrm{BCR}-\mathrm{ABL}$ oncogene has much higher ABL kinase activity.

\section{Role of Vitamin D in the Bone Marrow Microenvironment}

ALL is characterized by abnormal expansion of malignant lymphoblasts that replace hematopoietic stem cells (HSCs) from the bone marrow microenvironment (BMM) where they reside with a multitude of other cell types which help maintain homeostasis. HSCs tend to localize in two different niches in the bone marrow (BM), the perivascular niche and the endosteal niche (Nombela-Arrieta et al., 2013). Leukemia cells can migrate to the BMM similar to the HSCs (Peled et al., 1999), and can further disrupt the healthy microenvironment in the BM by creating a leukemia niche. This niche helps leukemia cells to proliferate better and hide from treatment and immune response (Fujisaki et al., 2011) (Nakasone et al., 2012).

The cells in the niche produce various cytokines and cell adhesion molecules that support lymphocyte proliferation and migration. Lymphocytes are attracted by a chemokine called stromal cell-derived factor 1(SDF-1 or CXCL12) which is produced by various cells in the BMM (Peled et al., 1999; van den Berk et al., 2014). CXCL12 binds to CXCR4, a receptor expressed on the lymphocytes and attract them towards the BMM. This CXCR4-CXCL12 axis has been shown to be important in homing of ALL cells towards the BMM (Colmone et al., 2008; Juarez et al., 2007; Sipkins et al., 2005). Various cell types such as perivascular stromal cells, mesenchymal stem cells (MSCs), endothelial cells, osteoblasts, fibroblasts secrete CXCL12 (although to various degrees) to regulate HSC maintenance (L. Ding, Saunders, Enikolopov, \& Morrison, 2012). Apart from these, CXCL12 abundant reticular cells (CARs) which can differentiate into 
osteoblasts and adipocytes also produce a significant amount of CXCL12 to help maintain HSC self-renewal, proliferation, and migration (Omatsu et al., 2010).

Human marrow-derived stromal cells (hMSCs) or mesenchymal stem cells include progenitors of several lineages including osteoblasts, chondrocytes and adipocytes (Krebsbach, Kuznetsov, Bianco, \& Robey, 1999; Prockop, 1997). Studies show that treatment of hMSCs with $1,25-(\mathrm{OH})_{2} \mathrm{VD}_{3}$ resulted in increased differentiation of hMSCs towards osteoblasts (P. Liu, Oyajobi, Russell, \& Scutt, 1999). Therefore, we investigated if vitamin D status altered the expression of CXCL12, produced by these cells in the BMM which can play a huge role in proliferation and homing of the leukemia cells.

Vitamin D is well known to play an important role in bone homeostasis. In diseases such as pancreatitis and hepatic stellate cancer, vitamin D was shown to remodel the stromal cells with reduced tumor supportive signaling (N. Ding et al., 2013) . Any changes in the bone marrow composition due to vitamin D deficiency can lead to bone marrow remodeling. Changes in expression of soluble factors such as chemokines, adhesion molecule in the bone could in turn alter the bone marrow microenvironment.

Considering $\mathrm{VD}_{3}$ deficiency causes a significant bone loss and decreased bone mineral density, it becomes critical to ascertain if vitamin D status can remodel the BMM. Here, we aimed to evaluate the changes associated with vitamin D deficiency on the bone marrow microenvironment. We investigated the effect of VD3 on both proportions of various BM cell types as well as expression of chemokines regulating tumor cell proliferation and homing to the BM that can ultimately impact the survival outcome.

\section{Central Hypothesis}

Vitamin D insufficiency or deficiency is common worldwide in the pediatric population, including those with leukemias. In addition, chemotherapies used to treat leukemia's such as glucocorticoids cause a further erosion of $\mathrm{VD}_{3}$ through induction of CYP3A4, that metabolizes 1,25- $(\mathrm{OH})_{2} \mathrm{VD}_{3}$ and 25-(OH) VD3. Given the prevalence of $\mathrm{VD}_{3}$ deficiency in the pediatric leukemia population, clinical trials are ongoing at multiple pediatric leukemia centers (Dana Farber Pediatric ALL, Children's Hospital LA) to determine whether $\mathrm{VD}_{3}$ supplementation can restore $\mathrm{VD}_{3}$ levels and help improve bone mineral density. However, there is a concern that $\mathrm{VD}_{3}$ treatment might cause a vitamin-drug interaction with CYP3A4 substrates because

- $\mathrm{VD}_{3}$ is known to transcriptionally induce intestinal CYP3A4 (Thummel et al., 2001)

- Intestinal CYP3A4 shows seasonal variation (Thirumaran et al., 2012) and 
- Schwartz JB et al., reported that $\mathrm{VD}_{3}$ supplementation increased atorvastatin (CYP3A4 substrate) clearance in VD 3 deficient patients (Schwartz, 2009).

Although many reports suggest an association between $\mathrm{VD}_{3}$ deficiency and poor survival outcome in various leukemia subtypes, there is no data on the potential effect of $\mathrm{VD}_{3}$ on survival of mice from pre-B cell leukemia.

Therefore, it is imperative to determine both the effect of $\mathrm{VD}_{3}$ on intestinal CYP3A expression and the pharmacokinetics of orally administered CYP3A4 metabolized leukemia therapies and its effect on the efficacy of those therapies on survival from acute lymphoblastic leukemia (ALL). Upon conclusion, we will understand the consequences of VD3 deficiency on survival outcome in a pre-B cell leukemia and interactions of $\mathrm{VD}_{3}$ with chemotherapeutics that are CYP3A4 substrates.

The central hypothesis of this proposal is "VD3 deficiency causes vitamin-drug interactions with antileukemic - CYP3A4 substrates, and also affects leukemia cell proliferation and migration leading to differential homing pattern causing an impact on survival outcome in BCR-ABL+ Arf-/- acute lymphoblastic leukemia". Below specific aims (Figure 2-1) were designed to evaluate this hypothesis.

\section{Specific Aim 1 (Chapter 3)}

a. Investigate the effect of $\mathrm{VD}_{3}$ status on expression of intestinal and hepatic mouse Cyp3a's, human CYP3A4 and other ADME genes in intestine, liver and kidney.

b. Determine if $\mathrm{VD}_{3}$ status alters the pharmacokinetics of orally administered antileukemic-CYP3A substrates, dexamethasone and dasatinib.

\section{Specific Aim 2 (Chapters 3 and 4)}

To evaluate the effect of $\mathrm{VD}_{3}$, alone or in combination with dexamethasone on survival of BCR-ABL+ Arf-/- ALL mouse model.

\section{Specific Aim 3 (Chapter 4)}

To determine the effect of $\mathrm{VD}_{3}$ on the bone marrow microenvironment remodeling, and BCR-ABL Arf-/- leukemic blast migration, homing and proliferation using in vivo and in vitro models. 


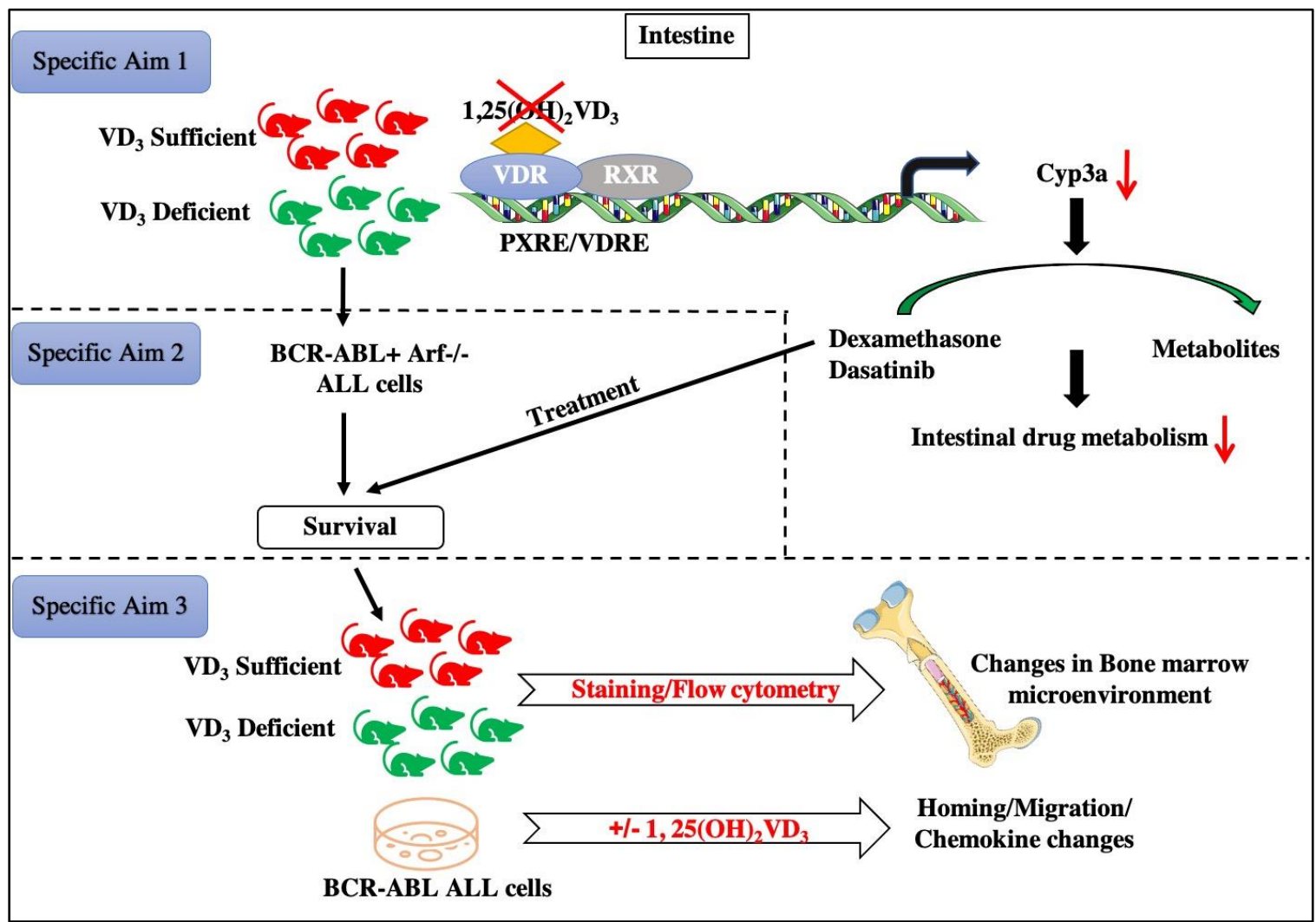

Figure 2-1. Schematic for specific aims. 


\section{CHAPTER 3. VITAMIN D STATUS ALTERS DRUG DETOXIFICATION GENE EXPRESSION AND DEXAMETHASONE INDUCTION OF CYTOCHROME P450S IN MICE}

\section{Introduction}

The Cyp3a subfamily of enzymes are responsible for the metabolic transformation of many drugs and some endogenous molecules. CYP3A4, an important member of Cyp3a family in humans can metabolize at least $50 \%$ of the prescription drugs and is found to be highly expressed in drug detoxification organs such as liver and small intestine (Kolars et al., 1994). Intestinal CYP3A-mediated first-pass metabolism of orally administered substrates such as cyclosporine, midazolam, lovastatin, felodipine, saquinavir, and buspirone is a major basis for their low oral bioavailability (Kolars, Awni, Merion, \& Watkins, 1991) (Paine et al., 1997).

Induction of hepatic and intestinal CYP3A4 is the basis for many drug-drug interactions. CYP3A4 is induced by nuclear hormone receptors in the NR1I family that are each activated by specific ligands: NR1I2/Pregnane X receptor (PXR) (e.g., glucocorticoids, rifampicin) and NR1I3/constitutive activated receptor (CAR) (e.g., phenobarbital) (Chai, Cherian, Wang, \& Chen, 2016) (Matsunaga et al., 2012) (Lin, 2006). We previously determined that CYP3A4 is also induced by NR1I1/Vitamin D receptor (VDR) activated by $1,25(\mathrm{OH})_{2} \mathrm{VD}_{3}$ (Thummel et al., 2001), although this induction occurs primarily in intestinal enterocytes where VDR is highly expressed versus hepatocytes where it is lowly expressed (Matsubara et al., 2008).

Several lines of evidence suggest vitamin D could cause a vitamin-drug interaction (VDI) with CYP3A4. (Lindh, 2011) showed in humans that systemic exposure of the immunosuppressant CYP3A4 substrates sirolimus and tacrolimus varied seasonally (higher in winter than summer), correlating with sunlight exposure and vitamin D levels. We showed that human intestinal biopsy CYP3A4 expression varied seasonally with sunlight and vitamin D levels (higher in Spring/Summer and lower in Fall/Winter) and corresponded with a higher systemic midazolam level in patients dosed in winter versus summer (Thirumaran et al., 2012). (Schwartz, 2009) showed that vitamin $\mathrm{D}$ deficient patients given vitamin D supplementation had increased clearance of the CYP3A4 substrate atorvastatin.

Vitamin D deficiency or insufficiency is common in patients with acute leukemias (Naz, R, T, \& Mahboob, 2013). Approximately $60 \%$ of acute lymphoblastic leukemia (ALL) patients at diagnosis are vitamin D insufficient or deficient, with deficiency and insufficiency more common in winter than summer (Young et al., 2018). Vitamin D deficiency in leukemia patients in combination with long term glucocorticoid therapy leads to reduction in bone mineral density (BMD) (Demirsoy et al., 2017). Some of these patients are prescribed $\mathrm{VD}_{3}$ supplementation to maintain $\mathrm{VD}_{3}$ levels and restore $\mathrm{BMD}$ (Orgel et al., 2017). Although intestinal Cyp3a expression can be regulated by VD3 and many chemotherapeutic agents are Cyp3a substrates, it has not yet been determined 
whether vitamin D sufficiency versus deficiency would affect the systemic concentrations of these chemotherapies. In this study we determined whether vitamin D sufficient versus deficient mice (a) differ in intestinal expression of VDR target genes, such as Cyp3a, or (b) differ in systemic exposure to the orally administered Cyp3a metabolized leukemia therapies DEX and dasatinib, or (c) differ in DEX's anti-leukemic efficacy.

\section{Methods}

\section{Animals}

Association for Assessment and Accreditation for Laboratory Animal Care (AALAC) guidelines were followed for all experimental animal procedures and housing, all protocols were approved by Institutional Animal Care and Use Committee (IACUC). E5-E6 pregnant C57BL/6J female mice were kept on the $\mathrm{VD}_{3}$ deficient (Harlan catalog no. 5A69, 0 IU of vitamin D) and $\mathrm{VD}_{3}$ sufficient diets, (catalog. No. 5BV8, $3.3 \mathrm{IU}$ of vitamin D) to develop vitamin D deficient and vitamin D sufficient mice (Figure 3-1), respectively. Pregnant dams were maintained on these diets throughout pregnancy, and after parturition, and pups were maintained on the corresponding diets after weaning as described in (Annu et al., 2020). The pups were age matched and both male and female, $\mathrm{VD}_{3}$ deficient and $\mathrm{VD}_{3}$ sufficient mice were used for Cyp3a expression, pharmacokinetic and efficacy studies. For BCR-ABL survival studies with DEX treatment, C57BL/6J sufficient mice were placed on $\mathrm{VD}_{3}$ deficient and $\mathrm{VD}_{3}$ sufficient diets beginning at weaning.

To observe the effect of vitamin D status on hCYP3A4 expression, cryopreserved hCYP3A4 transgenic $(\operatorname{tg})$ mouse sperm was used to re-derive the humanized CYP3A4 transgenic mouse model (Granvil et al., 2003). E5-E6 pregnant hCYP3A4-tg female mice were kept on $\mathrm{VD}_{3}$ deficient and sufficient diets throughout pregnancy and after parturition as described for C57BL/6J mice. Pups were maintained on the corresponding diets after weaning and were euthanized at 8 and 12 weeks of age for tissues, blood was collected by cardiac puncture and serum isolated and stored at $-80^{\circ} \mathrm{C}$ for $25-\mathrm{OH}-\mathrm{VD}_{3}$ analysis. Vitamin $\mathrm{D}$ deficiency in the mice was confirmed by analyzing serum $25(\mathrm{OH}) \mathrm{VD}_{3}$ levels in randomly selected mice in all studies (Table 1 ) and sent to the Veterinary Diagnostic Laboratory at Michigan State University (Lansing, MI) for 25$(\mathrm{OH}) \mathrm{VD}_{3}$ analysis. Intestine (duodenum), liver and kidney were harvested and stored in RNA later at $-20^{\circ} \mathrm{C}$.

\section{mRNA Quantification by Real-time PCR}

Mouse intestine (duodenum), liver and kidney tissues stored in RNA later at $20^{\circ} \mathrm{C}$ were used for RNA isolation. Trizol (Ambion Life technologies, CA, USA or Thermo Fisher scientific, CA, USA) was used to extract RNA according to the 


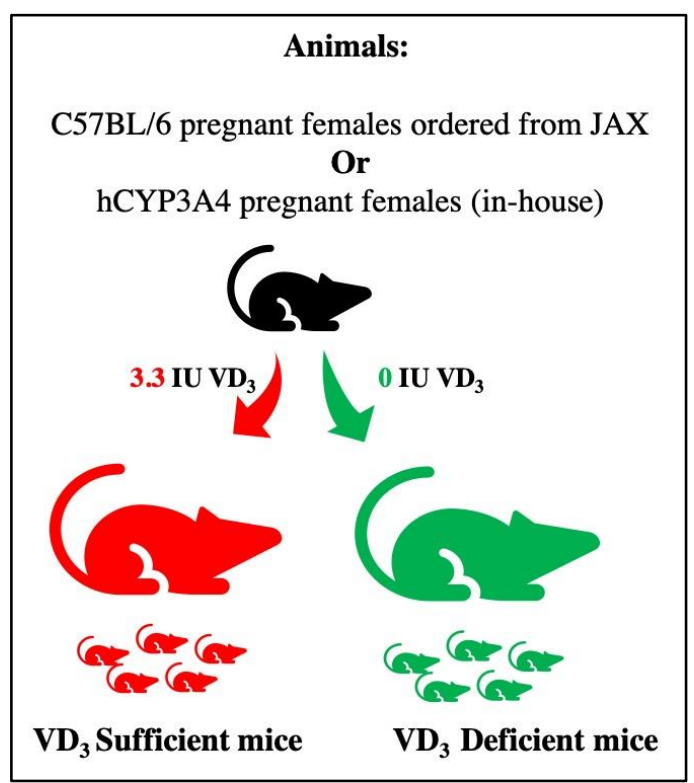

Figure 3-1. Design to develop age-controlled vitamin D-sufficient and -deficient mice. 
manufacturers protocol. Sterile Omni tips (OMNI International, GA, USA) were used to homogenize the tissue using Omni tissue homogenizer. $500 \mathrm{ng}$ of RNA was used to generate cDNA using the Thermoscript ${ }^{\mathrm{TM}}$ RT-PCR system (Invitrogen ThermoFisher, CA, USA). The cDNA was diluted to a total volume of $70 \mu \mathrm{L}$. Primers used for the amplification:

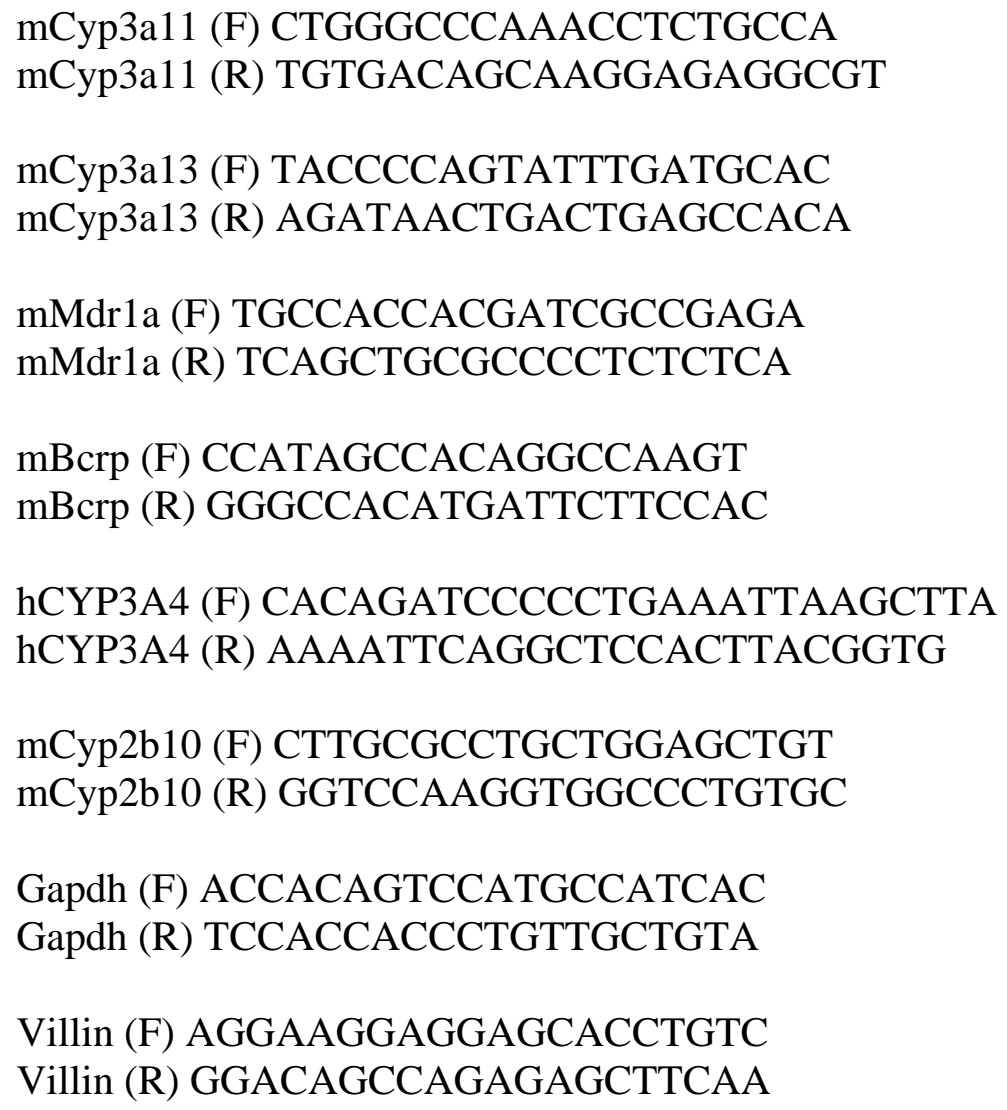

Real-time PCR quantification was performed using SYBR GreenER ${ }^{\mathrm{TM}}$ qPCR supermix for ABI PRISM Instrument (Life Technologies, Grand Island, NY) according to manufacturer's instructions. Samples were run at the following conditions: $95^{\circ} \mathrm{C}$ for 15 min, 40 cycles of $92^{\circ} \mathrm{C}$ for $30 \mathrm{sec}, 60^{\circ} \mathrm{C}$ for $30 \mathrm{sec}, 68^{\circ} \mathrm{C}$ for $1 \mathrm{~min}$ followed by a dissociation step. The relative amounts of mRNAs in each sample were normalized to Villin or Gapdh for intestine samples or Gapdh values for liver, kidney samples to control for quality of the mRNA. Comparative CT (ddCt) method was used to determine quantitative PCR values.

\section{Dexamethasone Pharmacokinetics}

\section{Dexamethasone administered in drinking water}

8-10-week-old C57BL/6 VD 3 Sufficient and VD $\mathrm{VD}_{3}$ deficient, male ( $\left.\mathrm{n}=8-13\right)$ and female $(n=5)$ mice were treated with the discontinuous DEX regimen as described in 
(Ramsey et al., 2015). $8 \mathrm{mg} / \mathrm{L}$ of dexamethasone sodium phosphate (Fresenius kabi LLC, Lake Zurich, IL, USA) is given in drinking water for 3.5 days followed by OFF dexamethasone but $600 \mathrm{mg} / \mathrm{L}$ of Sulfamethoxazole (Aurobindo Pharma Inc, Dayton, NJ, USA) and $120 \mathrm{mg} / \mathrm{L}$ of trimethoprim (Aurobindo Pharma Inc, Dayton, NJ, USA) for the next 3.5 days. $1 \mathrm{~g} / \mathrm{L}$ of tetracycline (Sigma-Aldrich Inc, St. Louis, MO, USA) was added during both phases. Assuming that mice drink $5 \mathrm{~mL}$ of water per day, the dose they receive would be 1.6-2 mg/kg/day (Ramsey et al., 2015). This discontinuous DEX ON/OFF schedule is then repeated with blood samples that were collected on day 0 (basal), and after each pulse of DEX i.e., day 3.5, 10.5 and 17.5 during the discontinuous DEX treatment (Figure 3-2). Blood was centrifuged at $10000 \mathrm{rpm}$ for 2 minutes and plasma was stored on dry ice. At the end of in vivo procedures, all plasma samples were transferred from dry ice and stored at $-80^{\circ} \mathrm{C}$ until analysis. DEX plasma concentrations were measured using liquid chromatography with tandem mass spectrometric detection (Ramsey et al., 2015).

\section{Single oral dexamethasone gavage}

The dosing and sampling scheme to assess DEX PK in mice after a single oral dose were performed as previously described (J. Li et al., 2018). Briefly, 14-week-old C57BL/6 VD3 sufficient and deficient male $(n=5)$ and female $(n=5)$ mice were given 2 $\mathrm{mg} / \mathrm{kg}$ dexamethasone sodium phosphate dissolved in $0.5 \%$ hydroxypropylmethylcellulose (HPMC) and $1 \%$ Tween 80 by oral gavage. Blood was collected via saphenous/retro-orbital vein at time points: $0.25,0.75,0.5,1,1.5,2,4,8,16$ hours after treatment (Figure 3-3). Samples were spun at $10000 \mathrm{rpm}$ for 2 minutes for plasma separation and stored at $-80^{\circ} \mathrm{C}$ until further analysis. DEX plasma concentrations were measured using liquid chromatography with tandem mass spectrometric detection (Ramsey et al., 2015).

\section{Dasatinib Pharmacokinetics}

The total plasma pharmacokinetics of dasatinib in $\mathrm{C} 57 \mathrm{BL} / 6 \mathrm{VD} 3$ sufficient and deficient mice were assessed after single oral gavage of $10 \mathrm{mg} / \mathrm{kg}$ of dasatinib free base equivalents (St. Jude Compound Management, SJ000518976-9, LC Labs, D-3307, Lot BDS-106, purity > 99\%) was dissolved in $80 \mathrm{mMol} / \mathrm{L}$ sodium citrate buffer $\mathrm{pH} 3.1$ for a total Dasatinib concentration of $1 \mathrm{mg} / \mathrm{mL}$ for a $10 \mathrm{~mL} / \mathrm{kg}$ gavage volume. Mice were grouped by vitamin D status and sex and studied at 8 and 10 weeks of age. Survival saphenous and facial bleeding of mice was conducted using IACUC-approved methods at $0.125,0.25,0.5,1,2,4,8,16$ and $24 \mathrm{hr}$. post dose, with 3 mice per time point (Figure 3-4). Each mouse was sampled 3 times on 2 separate occasions of dasatinib dosing. Blood was collected into a Sarstedt Minivette POCT $50 \mu \mathrm{L}$ K3 EDTA capillary device, dispensed into a microtube and vortexed to mix the anticoagulant. The tubes were then immediately centrifuged to plasma and stored on dry ice for the remainder of the study. At the end of the in vivo procedures, plasma samples were transferred from dry ice and 


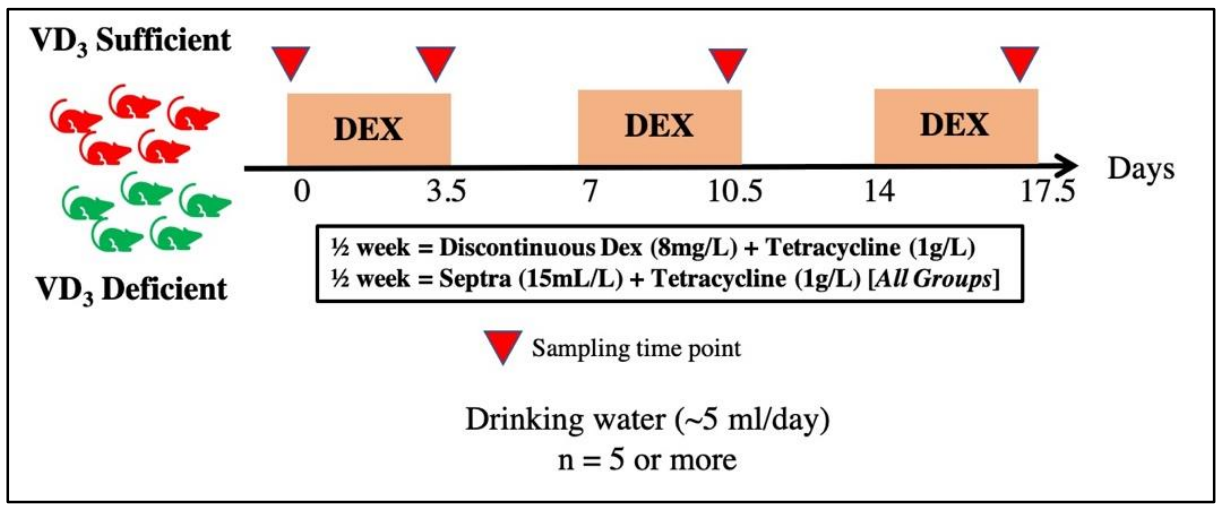

\section{Figure 3-2. Experimental design for pharmacokinetics of DEX using discontinuous DEX regimen in drinking water.}

Data source: Ramsey, L. B., Janke, L. J., Payton, M. A., Cai, X., Paugh, S. W., Karol, S. E., , . Relling, M. V. (2015). Antileukemic Efficacy of Continuous vs Discontinuous Dexamethasone in Murine Models of Acute Lymphoblastic Leukemia. PLoS One, 10(8), e0135134. doi:10.1371/journal.pone.0135134

\begin{tabular}{|c|c|c|c|c|c|c|c|c|c|c|}
\hline \multirow{8}{*}{$\mathrm{VD}_{3}$ Sufficient } & \multicolumn{10}{|c|}{ Dexamethasone (oral gavage): $2 \mathrm{mg} / \mathrm{kg}$} \\
\hline & 0.25 & 0.5 & 0.75 & 1 & 1.5 & \multicolumn{2}{|c|}{2} & 4 & 8 & 16 \\
\hline & $\boldsymbol{\Delta}$ & $\triangle$ & $\boldsymbol{\Delta}$ & $\boldsymbol{\Delta}$ & $\triangle$ & \multicolumn{2}{|c|}{$\boldsymbol{\Delta}$} & $\boldsymbol{\Delta}$ & $\boldsymbol{\Delta}$ & $\triangle \quad \mathrm{Hrs}$ \\
\hline & $\begin{array}{c}\text { Set } 1 \\
n=5\end{array}$ & $\begin{array}{c}\text { Set } 2 \\
n=5\end{array}$ & $\begin{array}{c}\text { Set } 3 \\
n=5\end{array}$ & $\begin{array}{c}\text { Set } 1 \\
n=5\end{array}$ & $\begin{array}{c}\text { Set } 2 \\
n=5\end{array}$ & \multicolumn{2}{|c|}{$\begin{array}{c}\text { Set } 3 \\
n=5\end{array}$} & $\begin{array}{c}\text { Set } 3 \\
n=5\end{array}$ & $\begin{array}{c}\text { Set } 1 \\
n=5\end{array}$ & $\begin{array}{c}\text { Set } 2 \\
\mathrm{n}=5\end{array}$ \\
\hline & & & Time $p$ & ints & 1st & 2nd & 3 rd & & & \\
\hline & & & Set & & 0.25 & 0.75 & 1.5 & & & \\
\hline & & & Set 2 & & 0.5 & 1 & 16 & & & \\
\hline & & & Set 3 & & 2 & 4 & 8 & & & \\
\hline
\end{tabular}

Figure 3-3. Experimental design for pharmacokinetics of DEX post oral DEX gavage $(2 \mathrm{mg} / \mathrm{kg})$.

Data source: Li, J., Chen, R., Yao, Q. Y., Liu, S. J., Tian, X. Y., Hao, C. Y., . . Zhou, T. Y. (2018). Time-dependent pharmacokinetics of dexamethasone and its efficacy in human breast cancer xenograft mice: a semi-mechanism-based pharmacokinetic/pharmacodynamic model. Acta Pharmacol Sin, 39(3), 472-481. doi:10.1038/aps.2017.153 


\begin{tabular}{|l|l|l|l|l|l|l|l|l|}
\hline VD & Sufficient & Dasatinib (oral gavage, $10 \mathrm{mg} / \mathrm{kg}$ ) & & & & & \\
\\
VD
\end{tabular}

\section{Figure 3-4. Experimental design for pharmacokinetics of dasatinib post oral} dasatinib gavage $(10 \mathrm{mg} / \mathrm{kg})$.

Data source: Furmanski, B. D., Hu, S., Fujita, K. I., Li, L., Gibson, A. A., Janke, L. J., . . . Baker, S. D. (2013). Contribution of ABCC4-mediated gastric transport to the absorption and efficacy of dasatinib. Clin Cancer Res, 19(16), 4359-4370.

doi:10.1158/1078-0432.CCR-13-0980 
placed at $-80^{\circ} \mathrm{C}$ until analysis. Dasatinib bioanalysis and pharmacokinetic analysis (see below) were performed by Preclinical Pharmacokinetic Shared Resource at St. Jude Children's Hospital and described in supplementary methods.

\section{Dasatinib bioanalysis}

Total plasma Dasatinib concentrations were assessed using a sensitive and specific liquid chromatography, tandem mass spectrometry assay. Dasatinib stock solutions were prepared in acetonitrile and used to spike matrix calibrators and quality controls. Protein precipitation was performed using a 1:4 ration of plasma to $15 \mathrm{ng} / \mathrm{mL}$ erlotinib $\mathrm{HCl}$ (St. Jude Compound Management, S00004053, Inventory ID:

SJCH0042996, Purity > 95\%) in methanol as an internal standard. A $3 \mu \mathrm{L}$ aliquot of the extracted supernatant was injected onto a Shimadzu LC-20ADXR high performance liquid chromatography system via a LEAP CTC PAL autosampler. The LC separation was performed using a Phenomenex Kinetex 2.6 um EVO C18 (100A, 50 x $2.1 \mathrm{~mm})$ maintained at $50 \mathrm{C}$ with gradient elution at a flow rate of $0.5 \mathrm{~mL} / \mathrm{min}$. The binary mobile phase gradient began with a linear increase to $100 \% \mathrm{~B}$ in 4.0 minutes. The column was then rinsed for 2.0 minutes at $100 \% \mathrm{~B}$ and then equilibrated at the initial conditions for two minutes for a total run time of eight minutes. Under these conditions, the analyte and IS eluted at 1.81 and 2.21 minutes, respectively. Analyte and IS were detected with tandem mass spectrometry using a SCIEX API 5500 Q-TRAP in the positive ESI mode with monitoring of the following mass transitions: Dasatinib 488.20 -> 401.00, erlotinib $\mathrm{HCl} 394.20$-> 250.10.

The experimental bioanalytical runs were all found to be acceptable for the purpose of a singlicate non-GLP, preclinical PK assessment. A linear model (1/X2 weighting) fit the calibrators across the 1.00 to $500 \mathrm{ng} / \mathrm{mL}$ range, with a correlation coefficient $®$ of $>=0.9058$. The lower limit of quantification (LLOQ), defined as a peak area signal-to-noise ratio of 5 or greater verses a matric blank with IS, was $1.00 \mathrm{ng} / \mathrm{mL}$. The intra-run precision and accuracy was $<11.6 \% \mathrm{CV}$ and $90.6 \%$ to $107 \%$ respectively.

\section{Pharmacokinetic analysis}

Plasma concentration-time $(\mathrm{Ct})$ data in $\mathrm{ng} / \mathrm{mL}$ for dasatinib were grouped by individual mouse, Vitamin D status, sex, and age, and were analyzed using nonlinear mixed effect (NLME) modeling as implemented in Monolix version 2018R1 (Lixoft SAS, Antony, France). Briefly, parameters and the Fisher Information Matrix (FIM) were estimated using the stochastic approximation expectation maximization (SAEM) algorithm, and the final log-likelihood estimated with importance sampling, all using the default Monolix initial settings, except that 1000 iterations were permitted for estimation of FIM using stochastic approximation. A variety of models were fit to the dasatinib $\mathrm{Ct}$ data, parameterized using apparent clearances, volumes of distribution, and absorption rate constant as needed. These models were assessed for goodness of fit using the -2-log likelihood (-2LL) value, Akaike and Bayesian Information Criterion (AIC, BIC), visual predictive checks, plots of model individual and population predicted versus observed 
data, residual plots, and the standard error of parameter estimates. A log-normal interindividual and inter-occasion parameter distribution was assumed on selected supported parameters, with both on- and off-diagonal elements of parameter covariance matrices tested. Additive and/or proportional error models were tested and implemented as supported. Beal's M3 method was used to handle any data that were below the LLOQ or above the upper limit of the assay range. The grouping levels were tested as categorical covariates on supported PK parameters, primarily the apparent oral clearance $(\mathrm{Cl})$. A covariate effect was considered significant if its addition reduced the -2LL by at least 3.84 units $(\mathrm{P}<0.05$, based on the $\chi 2$ test for the difference in the $-2 \mathrm{LL}$ between two hierarchical models that differ by 1 degree of freedom). Additionally, Wald test $\mathrm{P}$ values were outputted for each covariate effect by the Monolix software.

\section{Survival Study}

Murine BCR-ABL $\mathrm{Arf}^{-/-} \mathrm{Luc}^{+}$ALL cells were created by Dr. Charles Sherr (St. Jude Children's Research Hospital) and provided by Dr. Mary Relling (St. Jude Children's Research Hospital). Cells were cultured previously as described (Ramsey et al., 2014). C57BL/6 VD3 sufficient $(\mathrm{n}=24)$ and deficient $(\mathrm{n}=22)$ male mice $(8-18 \mathrm{wk}$ of age) were injected with 2,000 BCR-ABL $\mathrm{Arf}^{-1-} \mathrm{Luc}^{+} \mathrm{ALL}$ cells retro-orbitally on day zero. Body weights were not significantly different at the start of the study (day 0). Starting on day 4, mice were kept ON the discontinuous DEX dosing regimen (described above) where DEX was supplied in drinking water for 3.5 days, and then removed for the next 3.5 days, and this discontinuous DEX ON/OFF schedule was repeated until the animals died from leukemia (Figure 3-5). Xenogen IVIS-200 (Caliper Life Sciences, Hopkinton, MA) was used to acquire bioluminescent images 5 minutes post-intra peritoneal injection of $200 \mu \mathrm{L}$ of $100 \mathrm{mg} / \mathrm{kg}$ D-Luciferin (Caliper Life Sciences, Hopkinton, MA). Mice were anaesthetized with isoflurane during this procedure. The bioluminescent signal (photons/s) from a fixed region of interest (ROI) is used to monitor the disease progression twice every week. Images acquired here were analyzed using Living Image 3.1 software (Caliper Life Sciences), where whole body luciferase flux measurements (photons/s) was quantified as disease burden. Euthanasia was performed when the animal reached moribund stage which was determined based on hind limb paralysis, scruffy coat, lethargy or an inability to obtain food or water. We repeated the survival study with DEX treatment by employing pups from pregnant females placed on $\mathrm{VD}_{3}$ sufficient $(\mathrm{n}=13)$ and deficient $(\mathrm{n}=10)$ diets and observed similar effect that $\mathrm{VD}_{3}$ deficient mice with DEX treatment survived better than VD3 sufficient mice (data not included).

\section{Data Analysis}

Unpaired t-test or Mann-Whitney non-parametric tests were used to determine the significance in gene expression assays. Survival data was analyzed using Kaplan Meier 
Inject 2,000 BCR-ABL+ Arf-/-

ALL cells

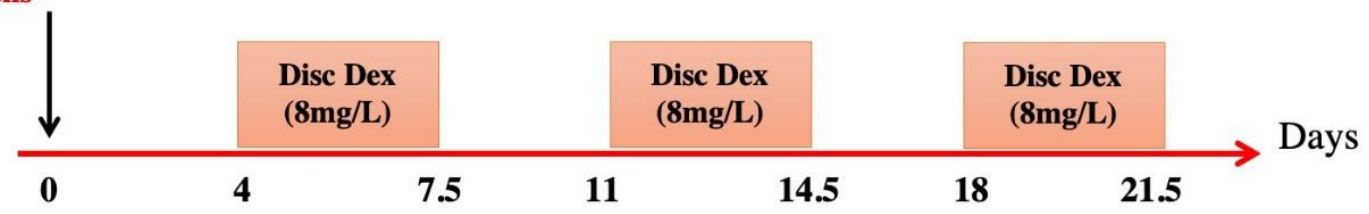

$1 / 2$ week = Discontinuous Dex $(8 \mathrm{mg} / \mathrm{L})+$ Tetracycline $(1 \mathrm{~g} / \mathrm{L})$

$1 / 2$ week $=$ Septra $(15 \mathrm{~mL} / \mathrm{L})+$ Tetracycline $(1 \mathrm{~g} / \mathrm{L})[$ All Groups $]$

Figure 3-5. Experimental design for survival of mice from BCR-ABL ALL placed on discontinuous DEX regimen in drinking water.

Data source: Ramsey, L. B., Janke, L. J., Payton, M. A., Cai, X., Paugh, S. W., Karol, S. E., .. . Relling, M. V. (2015). Antileukemic Efficacy of Continuous vs Discontinuous Dexamethasone in Murine Models of Acute Lymphoblastic Leukemia. PLoS One, 10(8), e0135134. doi:10.1371/journal.pone.0135134 
survival analysis and Gehan-Breslow-Wilcoxin test was used to determine significance. Significance is calculated at $\mathrm{p}<0.05$ and the Grubb's test on GraphPad QuickCals was used to remove any outliers.

\section{Results}

\section{Impact of Vitamin D Deficiency on Murine Duodenum Cyp3a and Transporter mRNA Expression}

Female C57BL/6 VD3 deficient mice had lower Cyp3a11 expression than VD3 sufficient mice, but the difference did not reach statistical significance (Figure 3-6B), while no difference in Cyp3a11 mRNA expression was observed between male $\mathrm{VD}_{3}$ sufficient versus deficient mice (Figure 3-6A). To better predict the effect of $\mathrm{VD}_{3}$ levels on human intestinal CYP3A4, we used a humanized transgenic mouse containing a bacterial artificial chromosome with the complete human CYP3A4 gene including all of its regulatory sequences (Cheung et al., 2006). Both mCyp3a11 and hCYP3A4 mRNA expression trended lower in male 8-week old (but not 12 week) $\mathrm{VD}_{3}$ deficient versus sufficient hCYP3A4-tg mice (Figure 3-6C). However, Cyp3a11 and hCYP3A4 expression were significantly lower in $\mathrm{VD}_{3}$ deficient female 12-week-old (but not 8 week) hCYP3A4-tg mice (Figure 3-6E, * $\mathrm{p}<0.05$ ). Because ABC efflux transporters play a major role in decreasing the oral bioavailability of DEX (Abcb1a/Mdr1a/Pglycoprotein) (Schinkel et al., 1995) and dasatinib (Mdr1a, Abcg2/Bcrp) (Hiwase et al., 2008) we evaluated their expression and found their mRNAs were significantly higher in the duodenums of $\mathrm{VD}_{3}$ deficient mice compared to sufficient mice (Figure 3-6C, $\mathbf{F}$, $* \mathrm{p}<0.05, * * \mathrm{p}<0.01)$. Vitamin D deficiency was confirmed in serum obtained from terminal blood samples by analysis of $25-(\mathrm{OH}) \mathrm{VD}_{3}$ in the control, vitamin $\mathrm{D}$ deficient mice (Table 3-1).

\section{Cyp and Transporter Expression in Liver and Kidney of $\mathrm{VD}_{3}-$ Sufficient and - Deficient hCYP3A4-tg Mice}

Because VDR can regulate expression of Cyp and transporters in other tissues important for drug disposition, we examined expression of these detoxification genes in kidneys and liver of mice with different vitamin D levels. In male mice, $\mathrm{VD}_{3}$ status had no significant effect on hepatic Cyp3a, while in females hCYP3A4 expression trended lower in VD 3 deficient mice (Figure 3-7A, B). Renal expression of hCYP3A4 was unchanged while Cyp24a1, a gene highly regulated by VDR (Kagi et al., 2018), was lower in both male and female $\mathrm{VD}_{3}$ deficient mouse kidney (Figure 3-7A, ${ }^{*} \mathrm{p}<0.05$ ). In liver hepatic Mdr1a and Mrp4 were higher in male VD 3 deficient mice (Figure 3-7C), but significantly lower in female $\mathrm{VD}_{3}$ deficient mice (Figure 3-7D, ${ }^{*} \mathrm{p}<0.05$, ${ }^{*} * \mathrm{p}<0.01$ ). Renal Mdr1a and Mrp4 were lower in VD3 deficient mice, while in female mice VD3 levels had no significant effect on expression of renal efflux transporters (Figure 3-7D). 


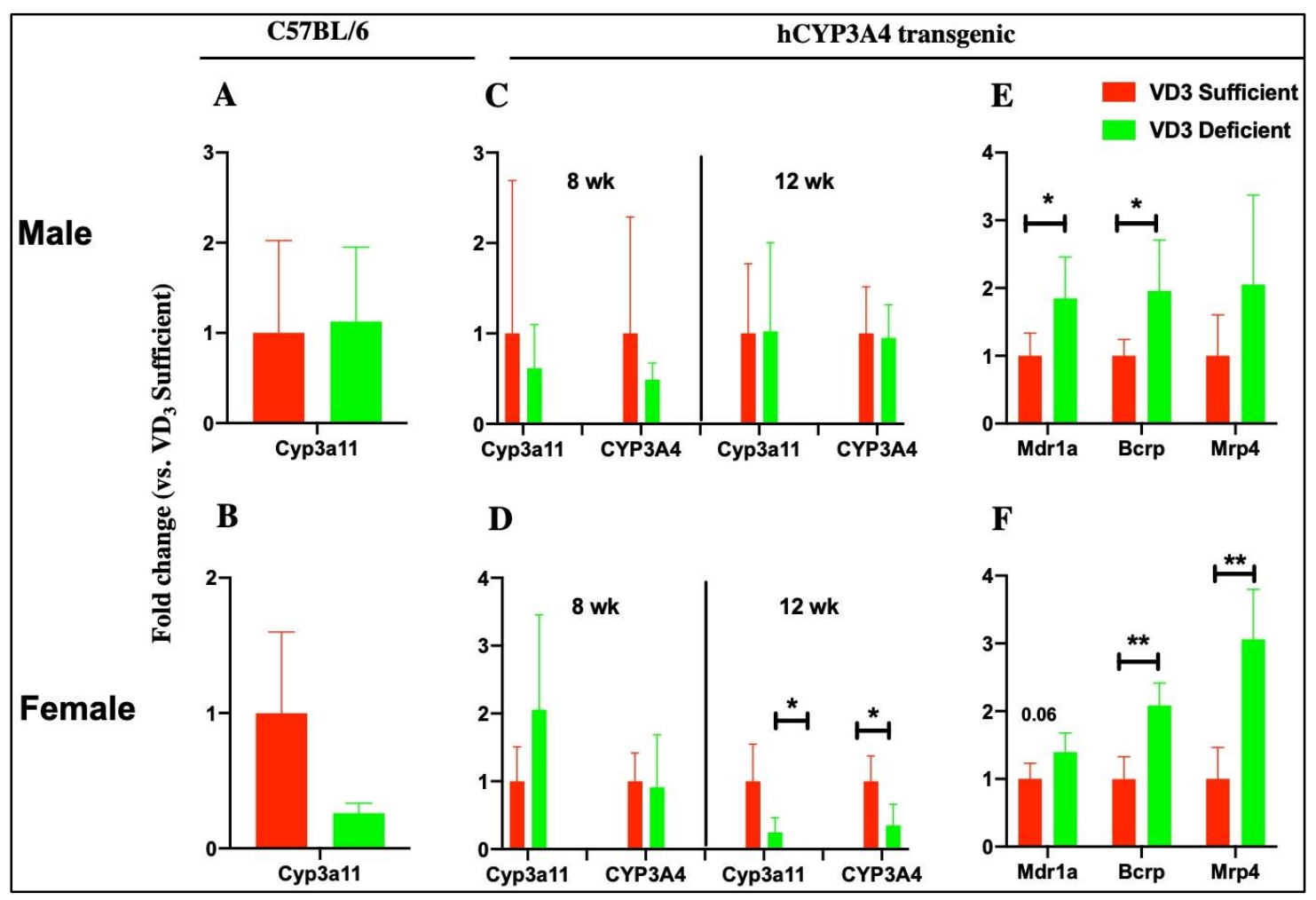

Figure 3-6. Cyp and transporter gene expression in duodenum of $\mathrm{VD}_{3}$ sufficient and deficient mice.

Notes: Influence of $\mathrm{VD}_{3}$ deficiency on intestinal Cyp3a11 expression in C57BL/6 (A) male, $n=3$ and (B) female, $n=3$ mice. Comparative expression of intestinal Cyp3a11, hCYP3A4, and drug efflux transporters in 8 and 12-week old hCYP3A4-tg (C, E) male and $(\mathrm{D}, \mathrm{F})$ female mice. Data represents the results from 4-6 independent mice per group in hCYP3A4-tg experiments and are expressed as mean (bars) + SE (error bars) fold change in mRNA expression in deficient mice (green bar) relative to each mRNAs expression in sufficient mice set as one (red bar). Villin was used as an internal control to normalize the target gene expression data. Mann-Whitney nonparametric test on GraphPad was used to determine significance between the groups $\left(* * p<0.01,{ }^{*} p<0.05\right)$. 
Table 3-1. Serum 25(OH) VD $\quad$ levels in $\mathrm{VD}_{3}$-sufficient and -deficient mice (Representative date).

\begin{tabular}{|c|c|c|c|}
\hline Sample name & Gender & $\begin{array}{c}25-(\mathrm{OH}) \\
\mathrm{VD}_{3}(\mathrm{nmol} / \mathrm{L})\end{array}$ & Diet \\
\hline C57BL/6 & $\mathrm{M}$ & 246 & $\mathrm{VD}_{3}$ sufficient \\
\hline C57BL/6 & M & 403 & $\mathrm{VD}_{3}$ sufficient \\
\hline C57BL/6 & $\mathrm{F}$ & 454 & $\mathrm{VD}_{3}$ sufficient \\
\hline hCYP3A4-tg & M & 297 & $\mathrm{VD}_{3}$ sufficient \\
\hline hCYP3A4-tg & M & 171 & $\mathrm{VD}_{3}$ sufficient \\
\hline hCYP3A4-tg & $\mathrm{F}$ & 221 & $\mathrm{VD}_{3}$ sufficient \\
\hline hCYP3A4-tg & $\mathrm{F}$ & 187 & $\mathrm{VD}_{3}$ sufficient \\
\hline C57BL/6 & M & 6 & $\mathrm{VD}_{3}$ deficient \\
\hline C57BL/6 & $\mathrm{M}$ & 17 & $\mathrm{VD}_{3}$ deficient \\
\hline C57BL/6 & M & 18 & $\mathrm{VD}_{3}$ deficient \\
\hline C57BL/6 & $\mathrm{F}$ & 7 & $\mathrm{VD}_{3}$ deficient \\
\hline C57BL/6 & $\mathrm{F}$ & 12 & $\mathrm{VD}_{3}$ deficient \\
\hline hCYP3A4-tg & M & 15 & $\mathrm{VD}_{3}$ deficient \\
\hline hCYP3A4-tg & M & 15 & $\mathrm{VD}_{3}$ deficient \\
\hline hCYP3A4-tg & $\mathrm{F}$ & 17 & $\mathrm{VD}_{3}$ deficient \\
\hline hCYP3A4-tg & $\mathrm{F}$ & 12 & $\mathrm{VD}_{3}$ deficient \\
\hline
\end{tabular}

Notes: $\mathrm{M}=$ Male, $\mathrm{F}=$ Female. 


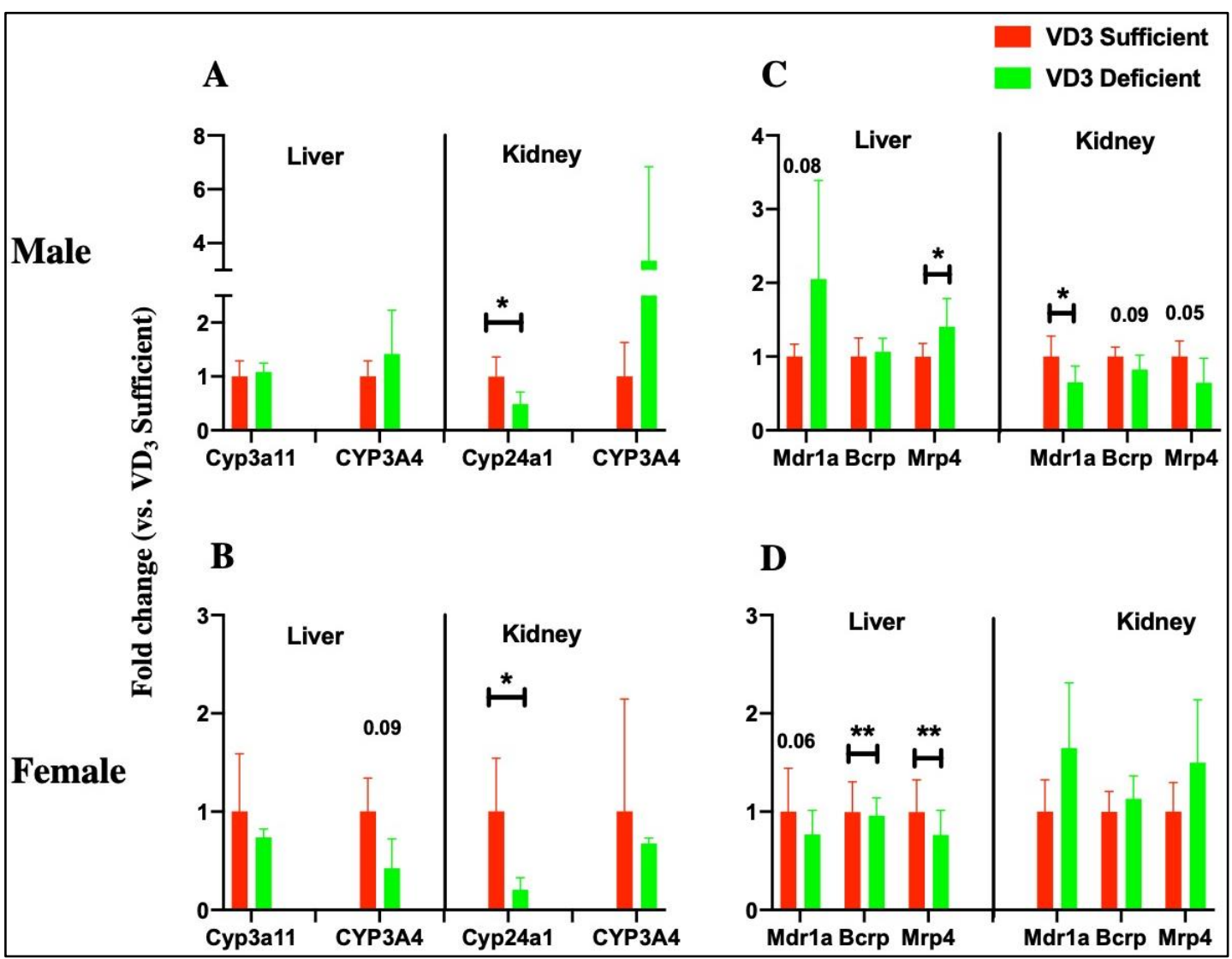

Figure 3-7. Cyp and transporter gene expression in liver and kidney of 12-week old hCYP3A4 mice.

Notes: Comparative expression of hepatic and renal Cyps and efflux transporters in 12week old hCYP3A4-tg (A, C) male and (B, D) female mice. Data was analyzed and graphed as described in Figure 3-6 legend. 


\section{Plasma Dexamethasone Concentrations in $\mathrm{VD}_{3}$ Deficient versus Sufficient C57BL/6 Mice}

Next, the effect of $\mathrm{VD}_{3}$ status on DEX systemic exposure was compared following a discontinuous oral DEX regimen, similar to that used to treat pediatric ALL patients, and that achieves plasma DEX concentrations $(2.6-201.5 \mathrm{nM})$ in mice similar to those achieved in pediatric patients on a discontinuous DEX regimen (Ramsey et al., 2015). Male $\mathrm{VD}_{3}$ deficient mice had significantly (Figure 3-8A, * $\mathrm{p}<0.05$ ) higher plasma DEX levels compared to sufficient mice with a mean of $31.7 \mathrm{nM}(\mathrm{n}=8)$ and $12.43 \mathrm{nM}$ $(\mathrm{n}=11)$, respectively, on day 3.5 , but no difference at later time points. A similar trend was observed in females (Figure 3-8B) with the mean plasma DEX concentration only different ( $63 \mathrm{nM}$ in deficient versus $52 \mathrm{nM}$ in sufficient mice) at an early time-point.

When we repeated the oral discontinuous DEX PK study in another 60 mice (15 per group) (Table 3-2) the DEX plasma concentrations were below the lower limit of assay detection in a majority of both male (80\%) and female $(68 \%) \mathrm{VD}_{3}$ sufficient mice from Days $3.5+10.5$, but was measurable in all female $\mathrm{VD}_{3}$ deficient mice and only below the limit of quantification in $8 \%$ of $\mathrm{VD}_{3}$ deficient female mice. This suggested that the higher intestinal Cyp3a in VD 3 sufficient versus deficient mice was impacting DEX systemic exposure, but only at the earliest exposure time points.

Because some of the variability in DEX concentrations between mice in all groups could have resulted from the mice drinking variable amounts of water, DEX pharmacokinetics was investigated following a single oral gavage of DEX $(2 \mathrm{mg} / \mathrm{kg})$ in both male and female $\mathrm{VD}_{3}$ sufficient and $\mathrm{VD}_{3}$ deficient mice. There was no significant difference between male or female $\mathrm{VD}_{3}$ sufficient versus deficient mice in their DEX systemic concentration at any single time point or in DEX area under the concentration curve (AUC) (Figure 3-9).

\section{Tissue-specific Impact of Dexamethasone on Intestinal and Hepatic CYP Expression in C57BL/6 VD 3 Sufficient versus VD 3 Deficient Mice}

Why was there an apparent time-dependent effect of VD3 status on DEX PK in mice (difference between groups seen only at early time points) on the discontinuous DEX regimen? We hypothesized that $\mathrm{VD}_{3}$ regulated changes in intestinal Cyp3a expression might be negated by prolonged treatment with DEX, that is also an inducer of Cyp3a. Therefore, we determined whether there was any difference in DEX induction of Cyp's in intestine and liver of $\mathrm{VD}_{3}$ sufficient versus VD 3 deficient mice. Cyp expression was analyzed in intestine and liver tissue from mice at day zero (no DEX) and on day 3.5 after a pulse of DEX using the oral discontinuous regimen. Duodenal Cyp3a11, Cyp3a13 and Cyp3a44 mRNAs were significantly induced (4-6-fold) (Figure 3-10A-C, *p<0.05) by DEX only in the $\mathrm{VD}_{3}$ deficient mice. In the liver Cyp2b10 was induced at least 50fold in both $\mathrm{VD}_{3}$ sufficient and $\mathrm{VD}_{3}$ deficient male and female mice, with no significant induction of hepatic Cyp3a's in any mice (Figure 3-10D-G, ${ }^{*} \mathrm{p}<0.05$ ). Thus, there were divergent 


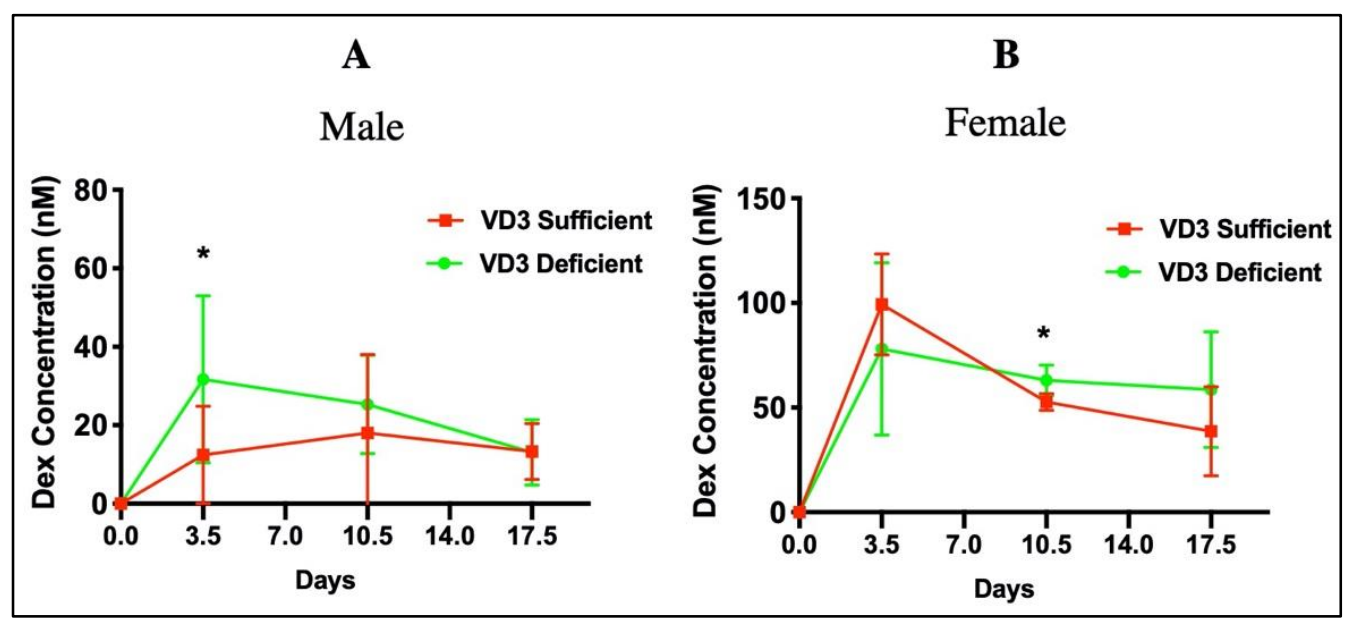

Figure 3-8. Plasma dexamethasone concentrations in C57BL/6 VD $\quad$ sufficient and deficient mice on a discontinuous dexamethasone regimen.

Notes: Plasma DEX concentrations on days 0 (basal), and at the end of each pulse of DEX i.e., days 3.5, 10.5 and 17.5 in (A) male ( $n=8-13$ /group), and (B) female $(n=5) V_{3}$ sufficient and VD3 deficient mice. The t-test on GraphPad was used to determine significance between the groups $(* \mathrm{p}<0.05)$.

Table 3-2. Repeat dexamethasone PK in drinking water.

\begin{tabular}{llccc}
\hline Group & Sex & Day 3.5 & Day 10.5 & Day 17.5 \\
\hline VD3 sufficient & M & $5(10 / 15$ BLQ $)$ & $0(10 / 10$ BLQ $)$ & 5 \\
VD3 deficient & M & $13(2 / 15$ BLQ $)$ & 10 & 5 \\
VD3 sufficient & F & $5(10 / 15$ BLQ $)$ & $3(7 / 15$ BLQ $)$ & 5 \\
VD3 deficient & F & 15 & 10 & 5 \\
\hline
\end{tabular}

Notes: Number of samples in each group with dexamethasone plasma levels that were measurable or below the lower limit of quantification (BLQ). $\mathrm{M}=$ Male, $\mathrm{F}=$ Female. 


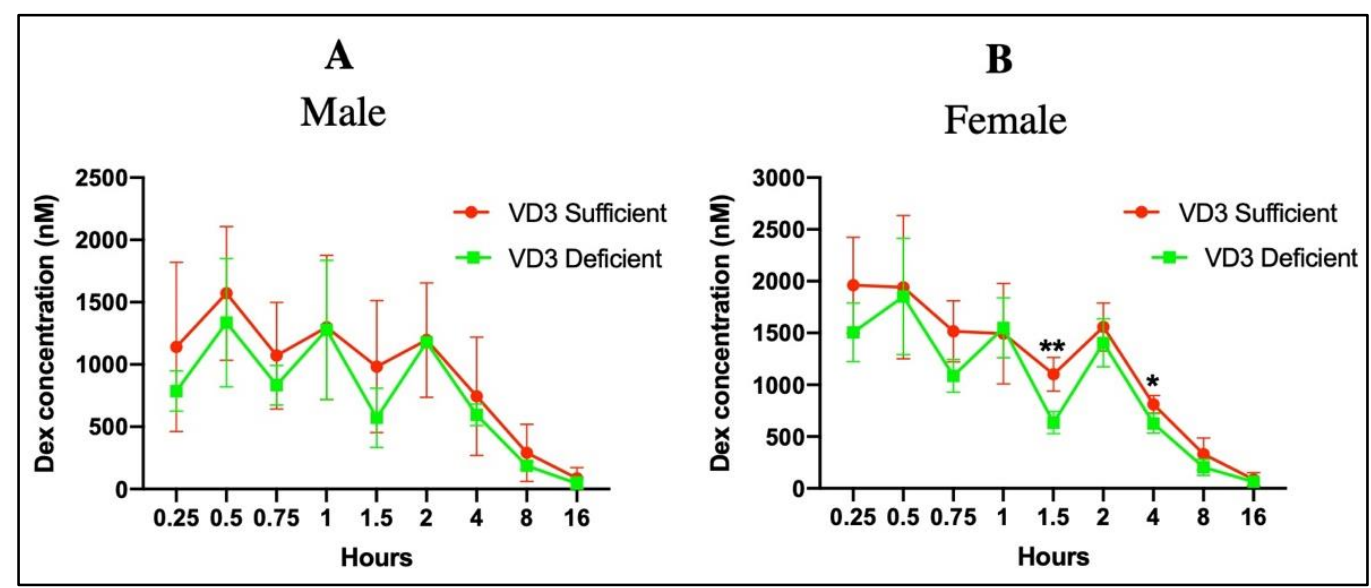

Figure 3-9. Plasma DEX pharmacokinetics in $\mathrm{VD}_{3}$-sufficient and -deficient mice after a single oral gavage of $2 \mathrm{mg} / \mathrm{kg}$ dexamethasone.

Notes: Plasma DEX concentrations in $\mathrm{VD}_{3}$-sufficient and -deficient 14-week (A) male and (B) female mice. A t-test was used to determine the significance of differences between $\mathrm{VD}_{3}$-sufficient and -deficient mice. 


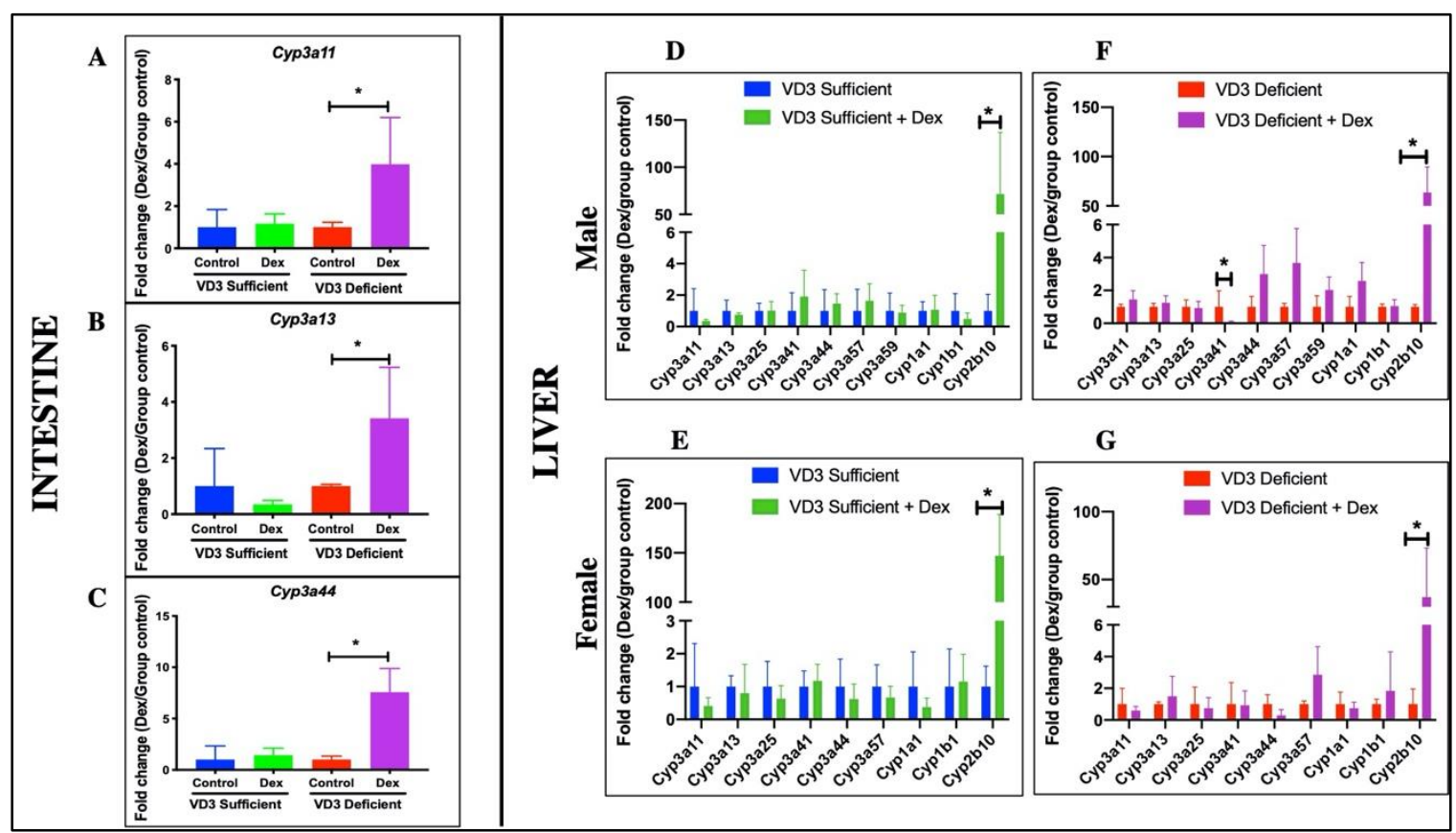

Figure 3-10. Induction of duodenal and hepatic Cyps in $\mathrm{VD}_{3}$-deficient mice after dexamethasone treatment.

Notes: Cyp3a mRNA expression was analyzed in VD3-sufficient and -deficient C57BL/6 male mice at time zero and following Dex in drinking water for 3.5 days, $n=3-5$ per group: Intestinal (A) Cyp3a11, (B) Cyp3a13, (C) Cyp3a44, and hepatic Cyps in VD3sufficient and -deficient (D, F) male and $(E, G)$ female mice $(n=3-5$ per group). Gapdh was used as an internal control to normalize the target gene expression data. MannWhitney nonparametric test on GraphPad was used to determine significance between the groups $(* * \mathrm{p}<0.01, * \mathrm{p}<0.05)$. 
effects of DEX on induction of Cyp3a's and Cyp2b that was both tissue-specific: DEX induction of Cyp3a was limited to $\mathrm{VD}_{3}$ deficient mice in intestine, and $\mathrm{DEX}$ induction of Cyp2b was observed in liver. A model is proposed in the discussion to account for these differential responses.

\section{Dasatinib Pharmacokinetics (Oral Gavage) in C57BL/6 Mice}

The plasma PK of dasatinib (a drug given throughout therapy to treat BCR-ABL+ acute lymphoblastic leukemias) was evaluated after two separate single oral gavage doses of $10 \mathrm{mg} / \mathrm{kg}$ to determine the effects of $\mathrm{VD}_{3}$ status, sex and age (8 wk versus $10 \mathrm{wk}$ ) on plasma PK using a nonlinear mixed effects PK modeling approach. Blood samples were collected at $0.125,0.25,0.5,1,2,4,8,16,24 \mathrm{hr}$. post-dose with $\mathrm{N}=3$ mice/group/time point and assayed using LC-MS. There was no influence of $\mathrm{VD}_{3}$ status or sex or age on oral dasatinib PK (Figure 3-11, *p<0.05). However, in males at both 8 and 10-weeks age at the $0.25 \mathrm{hr}$ time point, $\mathrm{VD}_{3}$ deficient mice had significantly higher plasma dasatinib concentrations than $\mathrm{VD}_{3}$ sufficient mice (Figure 3-11A,C).

The oral dasatinib plasma PK in the studied mice was best described using a linear, two-compartment, zero order absorption model, with proportional residual error. Inter-individual variability upon apparent oral systemic clearance $(\mathrm{Cl})$ and apparent volume of distribution of the central compartment (V1) was supported, improved the model fit and performance, as did the off-diagonal correlation between these two parameters. This was defined as the Base model. Inter-occasion variability on either $\mathrm{Cl}$ or V1 was not supported, resulted in model instability (failure of FIM convergence via SAEM) and a poorer model fit. This prevented formal testing of age as a covariate but implies that dasatinib PK is not significantly different between 8 and 10-week-old mice. Vitamin D sufficiency and sex were then tested alone and combined as covariates on $\mathrm{Cl}$ and were also found to be insignificant at the predefined $p<0.05$ threshold level. Overall, the Base model without any covariates performed the best, and suggested no influence of vitamin D status, sex, or age on oral dasatinib PK in mice.

\section{Effect of Vitamin D Status on Anti-leukemic Efficacy of Dexamethasone}

We recently found that $\mathrm{VD}_{3}$ deficient mice survived longer with BCR-ABL Arf-/ALL than sufficient mice in the absence of chemotherapy. We now compared survival from BCR-ABL Arf-/- ALL between $\mathrm{VD}_{3}$-sufficient and -deficient mice with the oral discontinuous DEX regimen. Leukemia was detected starting at day 19 in multiple $\mathrm{VD}_{3}$ sufficient mice but only in a few $\mathrm{VD}_{3}$ deficient mice (Figure 3-12). $\mathrm{VD}_{3}$ sufficient mice began to succumb to tumor burden on day $26 . \mathrm{VD}_{3}$ deficient mice with DEX treatment survived significantly (Figure 3-13, ${ }^{*} \mathrm{p}<0.05$ ) longer (median survival time 59 days) compared to the DEX-treated $\mathrm{VD}_{3}$ sufficient mice (median survival time 36 days). The proportion of total mice surviving BCR-ABL ALL at various time points (Table 3-3) further showed that even with DEX therapy $\mathrm{VD}_{3}$ sufficient mice had poorer survival than 


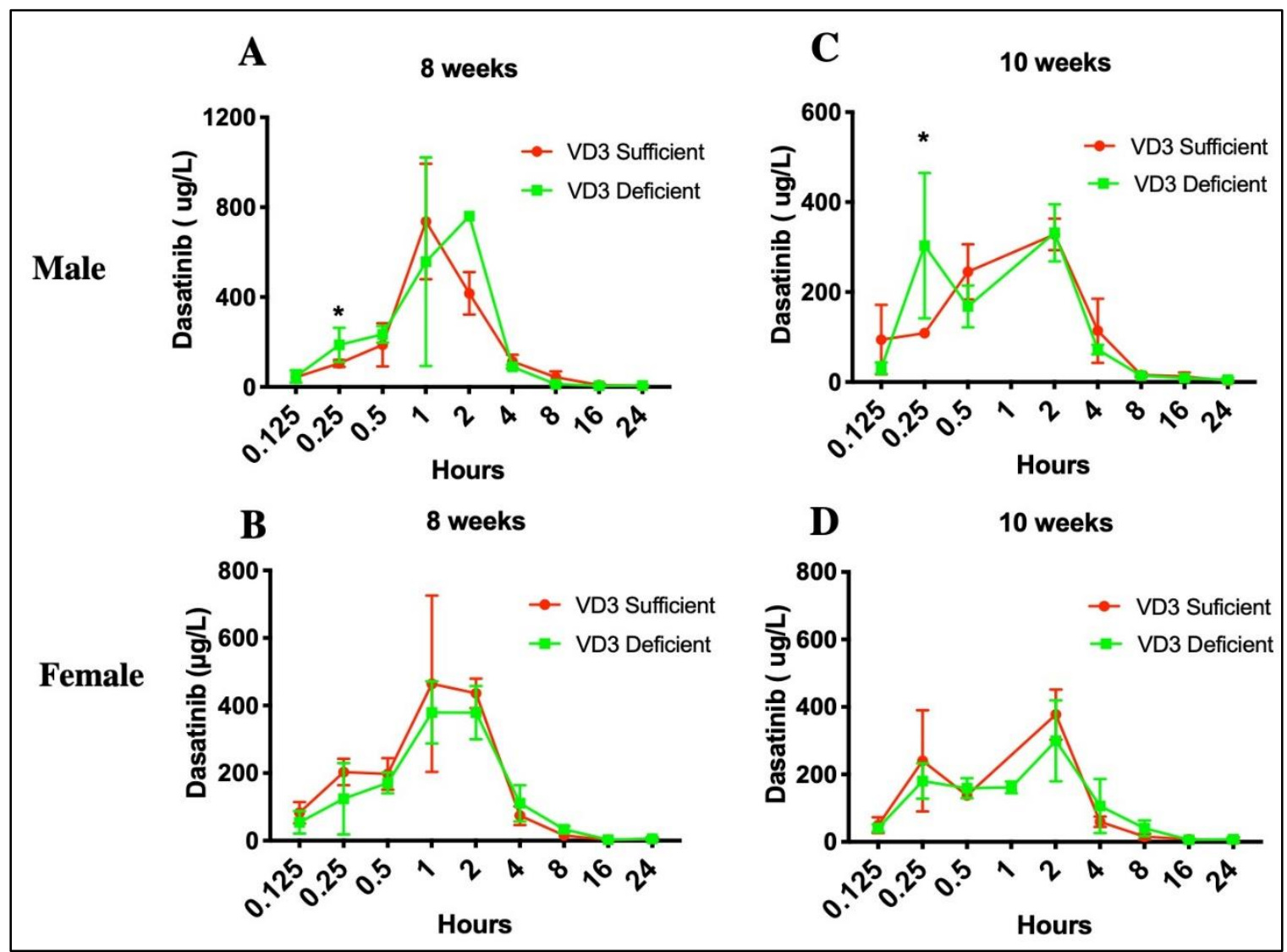

Figure 3-11. Plasma dasatinib pharmacokinetics in $\mathrm{VD}_{3}$-sufficient and -deficient mice after a single oral gavage of $10 \mathrm{mg} / \mathrm{kg}$ dasatinib.

Notes: Plasma dasatinib concentrations in $\mathrm{VD}_{3}$-sufficient and -deficient 8-week (A) male and (B) female mice, and 10-week (C) male and (D) female mice. A t-test was used to determine the significance of differences between $\mathrm{VD}_{3}$-sufficient and -deficient mice. 


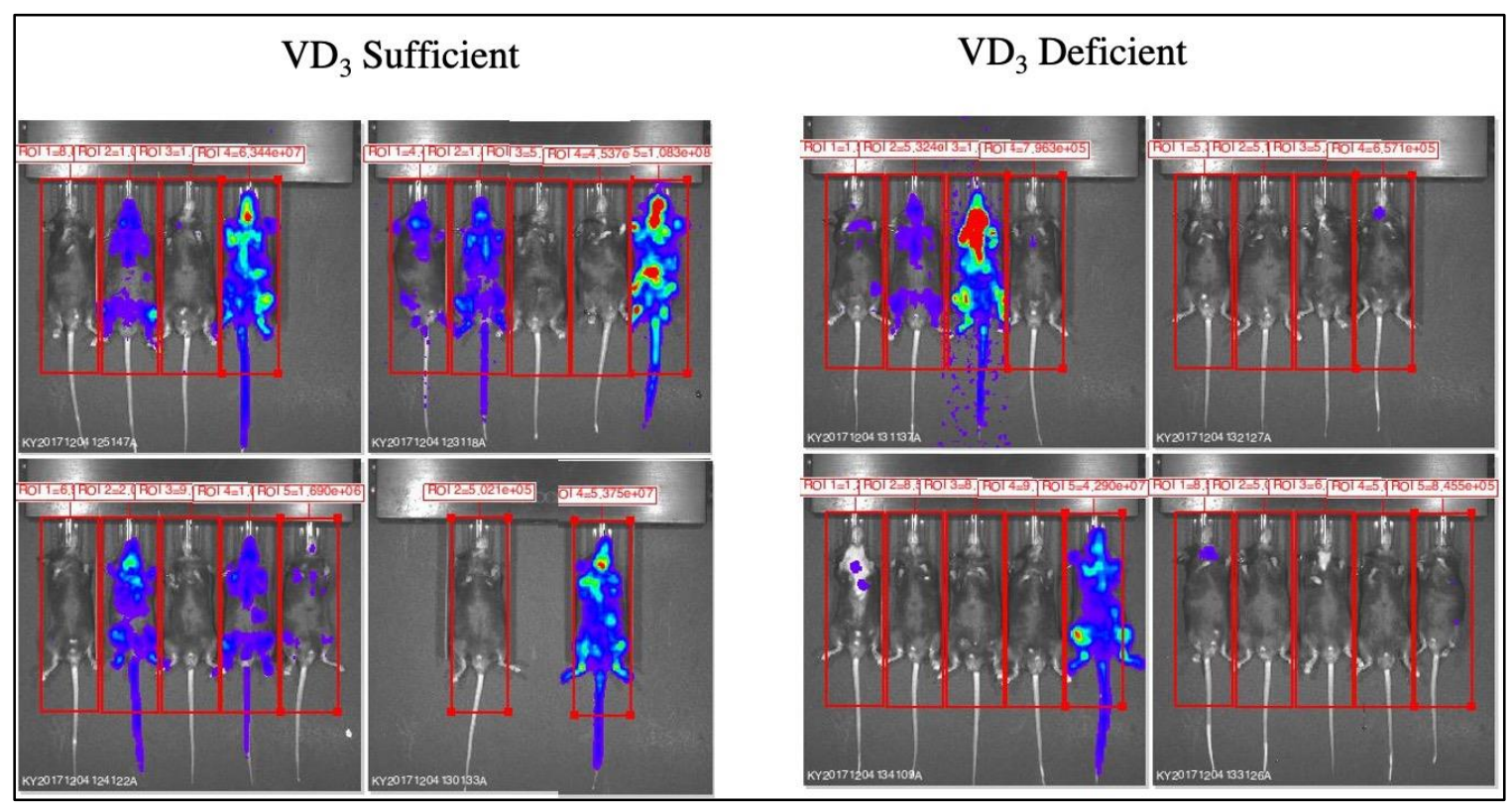

Figure 3-12. Xenogen images tracking disease burden in $V_{3}$-sufficient and deficient male mice injected with BCR-ABL ALL.

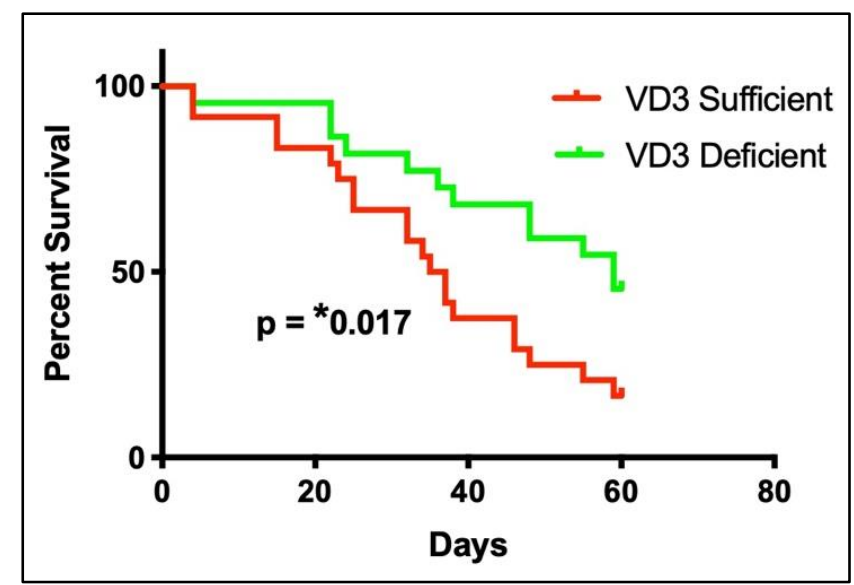

Figure 3-13. Kaplan-Meier curves for the effect of $\mathrm{VD}_{3}$ sufficiency vs. $\mathrm{VD}_{3}$ deficiency on survival of mice from BCR-ABL Arf-/- leukemia.

Notes: Kaplan Meier survival curve plotted for male $\mathrm{VD}_{3}$ sufficient and $\mathrm{VD}_{3}$ deficient mice with BCR-ABL Arf-/- leukemia and treated with a discontinuous dexamethasone regimen. Prism (version 8) was used to plot and run statistical analysis. The GehanBreslow-Wilcoxon test was used to find differences between survival curves of $\mathrm{VD}_{3}$ sufficient and -deficient groups $(* \mathrm{p}<0.05)$ 
Table 3-3. Survival proportions of $\mathrm{VD}_{3}$-sufficient and -deficient mice treated with dexamethasone during survival study from BCR-ABL.

\begin{tabular}{|c|c|c|}
\hline Days survived & $V_{3}$ sufficient & $V_{3}$ deficient \\
\hline 0 & 100.00 & 100.00 \\
\hline 4 & 91.67 & 95.45 \\
\hline 15 & 83.33 & \\
\hline 22 & 79.17 & 86.36 \\
\hline 23 & 75.00 & \\
\hline 24 & & 81.82 \\
\hline 25 & 66.67 & \\
\hline 32 & 58.33 & 77.27 \\
\hline 34 & 54.17 & \\
\hline 35 & 50.00 & \\
\hline 36 & & 72.73 \\
\hline 37 & 41.67 & \\
\hline 38 & 37.50 & 68.18 \\
\hline 46 & 29.17 & \\
\hline 48 & 25.00 & 59.09 \\
\hline 55 & 20.83 & 54.55 \\
\hline 59 & 16.67 & 45.45 \\
\hline
\end{tabular}


$\mathrm{VD}_{3}$ deficient mice. In sum, even with DEX chemotherapy, $\mathrm{VD}_{3}$ sufficient mice have a worse survival outcome than $\mathrm{VD}_{3}$ deficient mice from BCR-ABL ALL (Figure 3-13). No significant differences in serum chemistry except for lower high-density lipoprotein (HDL) levels in $\mathrm{VD}_{3}$ deficient mice was observed (Figure 3-14).

\section{Discussion}

There are at least two clinical trials (Dana Farber and Children's Hospital of LA) (ClinicalTrials.gov Identifier number: NCT01574274 and NCT01317940, respectively) administering vitamin $\mathrm{D}$ to insufficient and deficient pediatric acute lymphoblastic leukemia patients to restore $\mathrm{VD}_{3}$ sufficiency. Since the biologically active metabolite of $\mathrm{VD}_{3}\left(1,25(\mathrm{OH})_{2} \mathrm{VD}_{3}\right)$ induces intestinal CYP3A4 in humans and Cyp3as in mice, and since $\mathrm{VD}_{3}$ supplementation can alter the pharmacokinetics of orally administered CYP3A4 substrates such as atorvastatin (Schwartz, 2009), we hypothesized that Cyp3as might be higher in the intestine of $\mathrm{VD}_{3}$ sufficient versus $\mathrm{VD}_{3}$ deficient mice and that could lead to increased metabolism, decreased systemic exposure, and decreased antileukemic efficacy of the orally administered chemotherapies. Because DEX and dasatinib are majorly metabolized by CYP3A4 in humans (Christopher et al., 2008) (Gentile, 1996) and are orally administered to patients orally during ALL therapy (Mitchell et al., 2005) (Foa et al., 2011), this study sought to determine whether there might be unanticipated vitamin $\mathrm{D}_{3}$ interactions with these orally administered CYP3A metabolized ALL therapies.

In the C57BL/6J mouse model, the effect of VD3 levels was intestinal specific $\mathrm{VD}_{3}$ sufficient female mice had higher levels of intestinal Cyp3a11 compared to $\mathrm{VD}_{3}$ deficient mice while hepatic Cyp3a11 remained unchanged. This result is relevant to humans because hCYP3A4 was also significantly lower in the intestine of the VD 3 deficient humanized hCYP3A4-transgenic mouse. Consistent with lower intestinal Cyp3a in $\mathrm{VD}_{3}$ deficient female mice we found serum concentrations of DEX administered by a discontinuous oral regimen were transiently higher at the earliest, but not later times of DEX therapy in these deficient mice. In contrast, when DEX was administered orally as a single oral bolus dose, there was no significant difference in the DEX AUC between the VD3 sufficient and deficient mice. It is possible that there was no difference in DEX AUC following the single oral DEX gavage because the gut concentration (Cgut) achieved $\sim 200 \mu \mathrm{M}(2 \mathrm{mg} / \mathrm{kg}$ into a gastric volume of $300-400 \mu \mathrm{L})$ might saturate Cyp3a (assuming mouse Cyp3a is similar to CYP3A4's Km of $\sim 25 \mu \mathrm{M}$ for $6 \beta$ - and $6 \alpha$ hydroxydexamethasone formation) (Gentile, 1996). In contrast, mouse intestinal Cyp3a would readily metabolize DEX from the drinking water assuming the DEX Cgut is $\sim 7.6-$ $10.1 \mu \mathrm{M}$ at any time-point (assume drinking $200-400 \mu \mathrm{l} / \mathrm{hr}$ ). Similar to the findings with dexamethasone, there was no influence of $\mathrm{VD}_{3}$ status on oral dasatinib PK.

While we chose to test the effect of $\mathrm{VD}_{3}$ levels on dexamethasone systemic exposure because it is an orally administered anti-leukemic therapy, we would not $a$ priori have predicted that intestinal dexamethasone CYP3A-mediated metabolism would be affected by $\mathrm{VD}_{3}$ levels for several reasons (Figure 3-15). First, DEX has a 


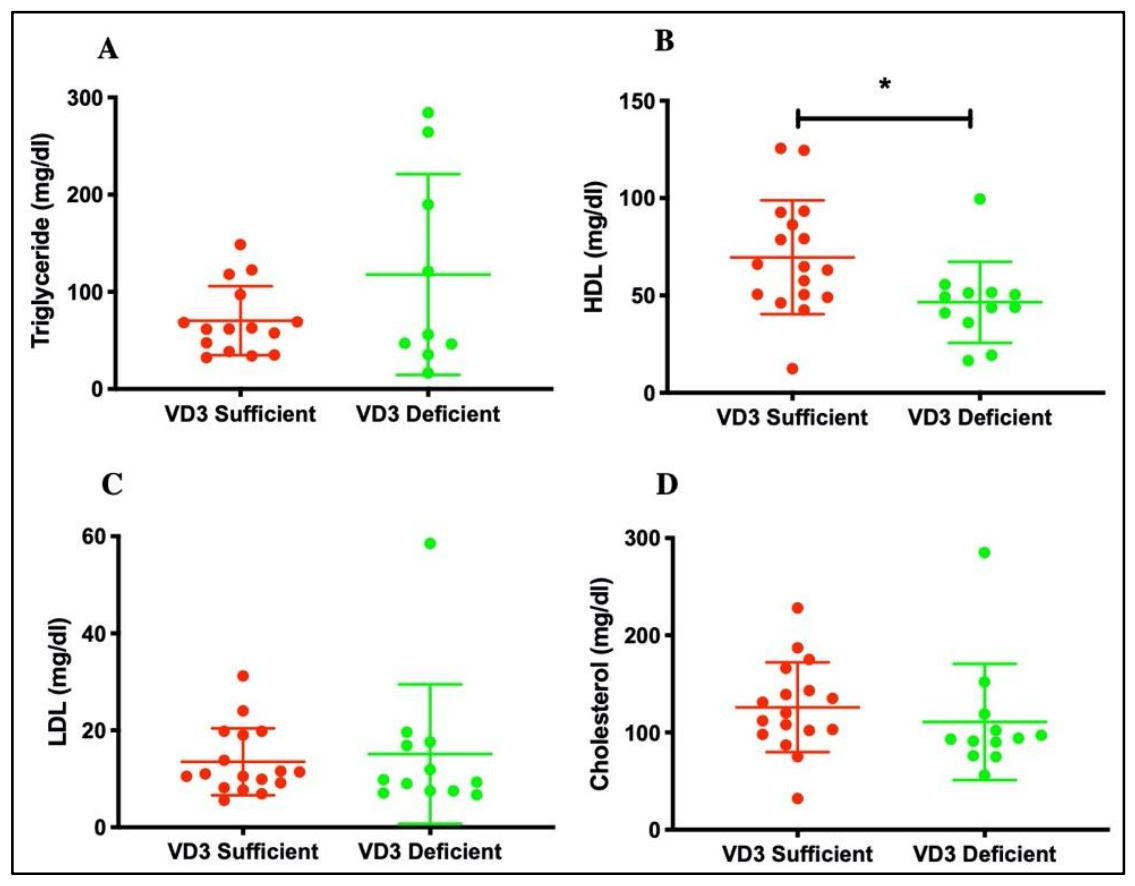

Figure 3-14. Serum chemistry of $\mathrm{VD}_{3}$-sufficient and -deficient mice.

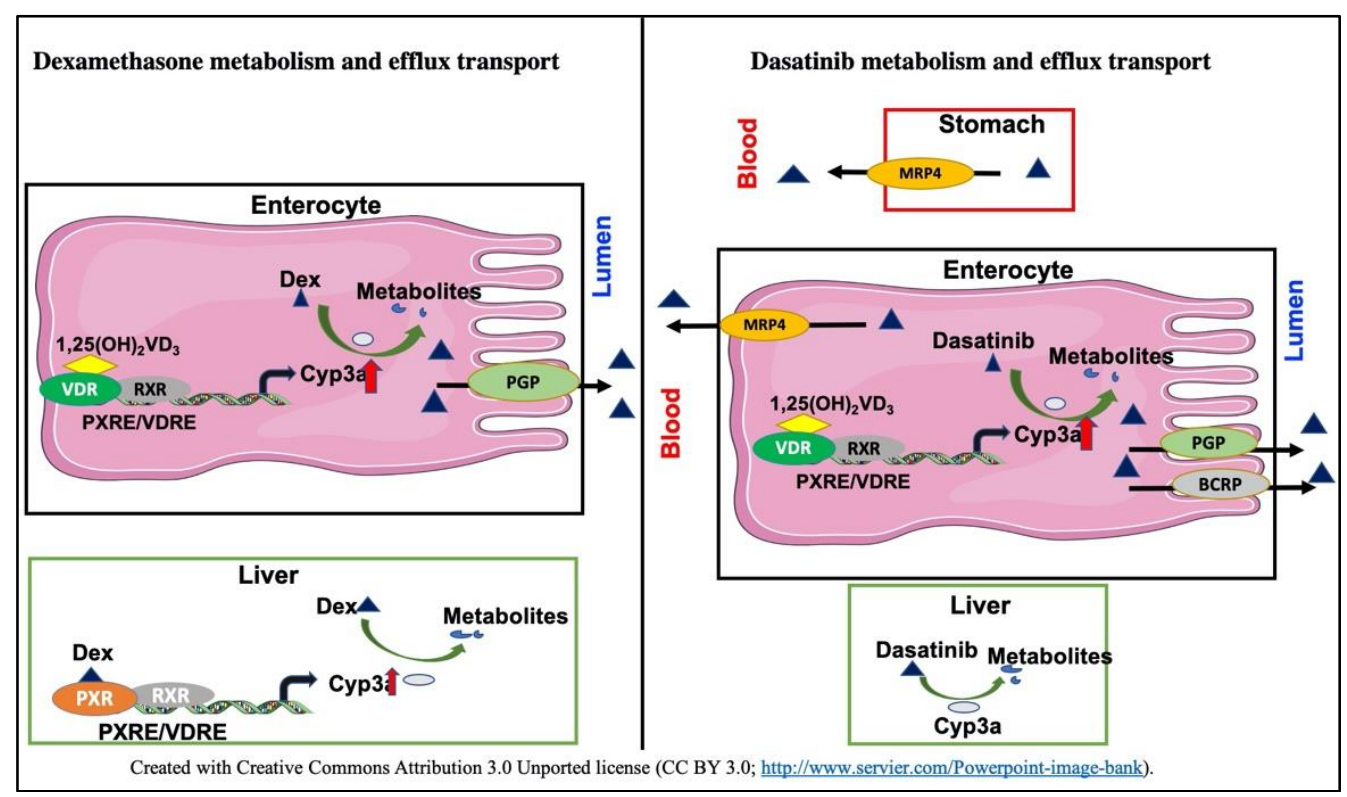

Figure 3-15. Cartoon depicting dexamethasone and dasatinib metabolism and efflux transport in intestine and liver. 
relatively high oral bioavailability in health individuals (76\% and $81 \%$ (Loew, Schuster, \& Graul, 1986)). Hence, even though DEX e can be metabolized by intestinal tissue (Siebe et al., 1993), CYP3A-mediated DEX first-pass metabolism in the gut is probably minimal. Second, the magnitude of DEX drug-drug interactions with CYP3A inducers and inhibitors (low-modest) supports this conclusion. Third, DEX is known to be primarily cleared by hepatic pathways (Gentile, 1996) undergoing extensive CYP3A4 dependent hepatic metabolism in vivo and in vitro (Tomlinson et al., 1997), and there was no effect of $\mathrm{VD}_{3}$ status on mouse hepatic Cyp3as consistent with the low levels of VDR in hepatocytes compared to intestine (Gascon-Barre et al., 2003). Hence, it was not clear that vitamin D induced variation in intestinal CYP3A4 expression would impact oral DEX systemic exposure.

We chose to test the effect of $\mathrm{VD}_{3}$ levels on dasatinib pharmacokinetics because this CYP3A substrate is given orally throughout therapy for BCR-ABL ALL. We would have predicted that dasatinib oral bioavailability might be affected by $\mathrm{VD}_{3}$ levels (Figure 3-15). Dasatinib is rapidly absorbed from the gastrointestinal tract after oral dosing, with the Cmax reached within 0.6 to $2 \mathrm{~h}$. Bioavailability of dasatinib is quite variable with one study showing 45-51\% bioavailability (Luo et al., 2006) and other indicating $17 \%$ in mice (Kamath, Wang, Lee, \& Marathe, 2008). Using bile duct cannulated rats, the percentage of unchanged drug in the gastrointestinal tract was 33\% suggesting 67\% was absorbed. However, the oral bioavailability in these rats was $27 \%$, which means $63 \%$ of drug did not reach the systemic circulation, possibly due to pre-systemic metabolism. Moreover, at least $40 \%$ of the total drug (or $60 \%$ of absorbed drug) might undergo hepatic metabolism. Dasatinib is primarily metabolized by CYP3A4. How much does intestinal CYP3A4 contribute to dasatinib metabolism? On the one hand coadministration of dasatinib with grapefruit juice is contraindicated (Squibb, 2006) suggesting there is intestinal CYP3A4 metabolism. Moreover, the magnitude of reported interaction with CYP3A inducers and inhibitors (large) supports the assumption of first pass effect at both intestine and liver. However, it should also be considered that CYP3A4-mediated dasatinib metabolism generates CYP3A4 mechanism-based inactivators with a $\mathrm{K}(\mathrm{I})=6.3 \mathrm{microM}$ and $\mathrm{K}$ (inact) $=0.034 \mathrm{~min}(-1)(\mathrm{X}$. Li et al., 2009). In our study dasatinib was dosed at $10 \mathrm{mg} / \mathrm{kg}$, the calculated Cgut is $1.5 \mathrm{mM}$, and the Cmax achieved was $2 \mu \mathrm{M}(0.002 \mathrm{mM})$. Hence, it is possible that intestinal CYP3A4 undergoes mechanism-based inhibition by CYP3A4-generated metabolites while hepatic CYP3A4 might not.

Is there an intestinal drug efflux transporter-mediated Vitamin $\mathrm{D}_{3}$ interaction with either dexamethasone or dasatinib? An additional variable affecting oral dexamethasone intestinal absorption could be intestinal Pgp (Schinkel et al., 1995). We first demonstrated that intestinal P-gp is highly inducible not only by drugs (Schuetz, Wrighton, Safe, \& Guzelian, 1986) but also by vitamin D exposure (Thummel et al., 2001). Hence, in theory variable VD3 levels could influence intestinal Pgp expression and contribute to variable DEX absorption and systemic exposure. Dasatinib is also a substrate of Mdr1a and Bcrp that are present at significant levels on the apical membrane of intestinal enterocytes and each has been shown to contribute to limiting dasatinib's oral bioavailability (Lagas et al., 2009) (Chen et al., 2009). Conversely, evidence that 
there was a significantly lower dasatinib AUC in Mrp4 KO versus WT mice supports Mrp4 enhanced dasatinib gastric absorption of (Furmanski et al., 2013). However, only Mdr1a has been shown to be induced in enterocytes treated with vitamin D (Thummel et al., 2001). However, not only did our study find no effect of $\mathrm{VD}_{3}$ levels in mice on dasatinib's AUC, nor any chronic effect on DEX systemic exposure, in fact the expression of Mdr1a mRNA was actually higher in the intestines of $\mathrm{VD}_{3}$ deficient versus sufficient mice, a paradoxical finding that others have also reported (Quach et al., 2018).

It is also possible that the paradoxical decrease in intestinal drug efflux transporters for dexamethasone (Pgp, Bcrp) and dasatinib (Pgp) in VD 3 Sufficient mice (Figure 3-15) negated the increased intestinal Cyp3a in these same mice. VD 3 deficient mice had significantly lower intestinal Cyp3a expression, but significantly higher Mdr1a levels. The combined opposing effects of metabolism and efflux transport on dexamethasone and dasatinib in this model may have ultimately erased any individual effect of $\mathrm{VD}_{3}$ on their metabolism or transport.

Why was DEX apparently more rapidly metabolized in $\mathrm{VD}_{3}$ deficient versus sufficient male only at only the earliest measured time-point in mice given an oral discontinuous dexamethasone regimen? We hypothesized that because DEX over time is inducing intestinal Cyp3a only in the $\mathrm{VD}_{3}$ deficient mice this is negating the difference in intestinal Cyp3a expression seen between $\mathrm{VD}_{3}$ deficient versus sufficient mice. The inability to induce Cyp3a in VD3 in sufficient mice was not because some theoretical maximum intestinal Cyp3a expression level has already been achieved in VD 3 sufficient mice because the threshold cycle (Ct value) for Cyp3a amplification was lower in VD3 deficient mice treated with DEX then in vitamin D sufficient mice with or without DEX (i.e., the relative expression level of Cyp3a in the DEX induced $\mathrm{VD}_{3}$ deficient mice was higher than the Cyp3a level in sufficient mice). Indeed, the selective DEX induction of intestinal Cyp3a in VD3 deficient mice, but not sufficient mice (Figure 3-10), suggests there is competition of VDR and PXR at the VDRE/PXRE binding sites in the Cyp3a promoter. VDR/NR1I1 and PXR/NR1I2, both members of the NR1I family of nuclear hormone receptors, share common DNA binding elements (Reschly \& Krasowski, 2006). We previously showed that VDR-RXR and PXR-RXR heterodimers bind to the identical DNA elements in the Cyp3a promoter (Thummel et al., 2001). Moreover, there is precedence for VDR-RXR and PXR-RXR heterodimers competing at common binding elements because PXR bound by the potent ligand rifampin was previously shown to inhibit VD3-ligand activated VDR binding to the CYP24 promoter in intestinal cells (C. Zhou et al., 2006). Results from this study suggest that in sufficient mice $\mathrm{VD}_{3}$ activated VDR-RXR bound to the Cyp3a VDRE/PXRE is preventing DEX (a relatively weak PXR agonist $(\mathrm{Kd} \sim 30 \mu \mathrm{M})$ )-activated PXR-RXR from displacing VDR from the VDRE/PXRE binding site (Figure 3-16). Conversely, in $\mathrm{VD}_{3}$ deficient mice the VDRE/PXRE is unoccupied by VDR because the $\mathrm{VD}_{3}$ ligand level is insufficient to ligand activate DNA binding, so the DEX -activated PXR-RXR can readily bind to and activate the Cyp3a promoter (Figure 3-16).

Tissue-specific DEX induction of Cyp3a in intestine but not liver, but DEX induction of Cyp2b in liver, suggests compartment specific induction of these Cyps 


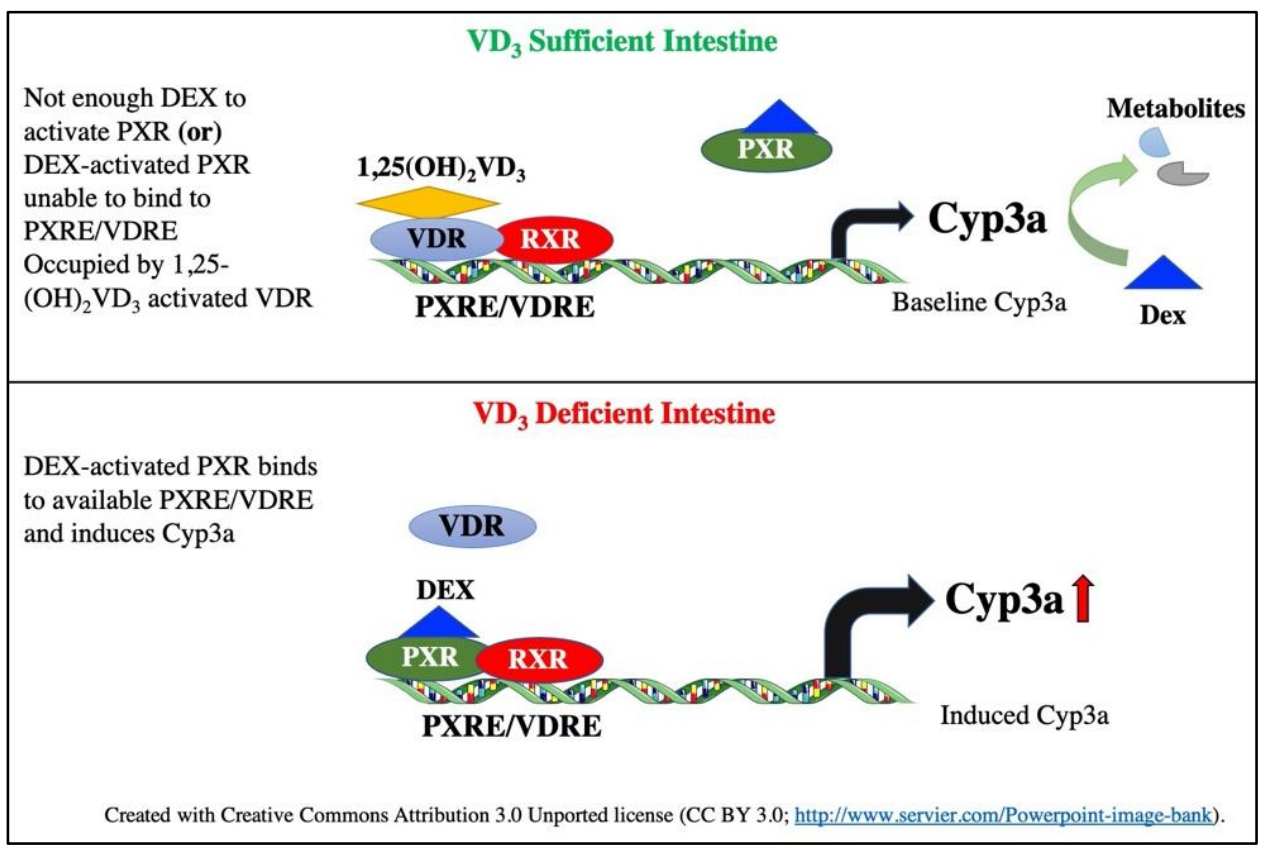

Figure 3-16. Cartoon depicting hypothesis that dexamethasone-activated PXR can induce Cyp3a in $\mathrm{VD}_{3}$ deficient mice, but not in $\mathrm{VD}_{3}$-sufficient mice where $1,25(\mathrm{OH})_{2} \mathrm{D}_{3}$-activated VDR blocks DEX-PXR induction. 
by PXR and the glucocorticoid receptor (Figure 3-17). The dose response curves for glucocorticoid activation of GR versus PXR are strikingly different (Figure 3-17). GR is ligand activated at $\mathrm{nM}$ concentration of glucocorticoids and makes physiological sense as these steroids need to be maintained within a strict homeostatic range. In contrast, the ED50 for PXR activation by glucocorticoids is approximately $30 \mu \mathrm{M}$ (Kliewer et al., 1998). Indeed, PXR requires stress/pathological levels and higher levels of glucocorticoids to be activated and then to induce Cyp3a and other detoxification pathways. If PXR were activated by glucocorticoids at nanomolar concentrations this would profoundly perturb endogenous glucocorticoid homeostasis. In contrast, Cyp2b is transcriptionally activated by the glucocorticoid receptor (GR) and we previously showed GR is required for DEX induction of Cyp2b in mice (Schuetz et al., 2000). Conversely, glucocorticoid induction of Cyp3a occurs via PXR with no participation of, or requirement for, GR (Schuetz et al., 2000). The observed systemic concentrations of DEX $(2.6-201.5 \mathrm{nM})$ in mice on the discontinuous regimen were in the nanomolar range which would activate GR, but not PXR, in liver. Accordingly, only Cyp2b was induced by DEX -activated GR in liver (Figure 3-17). However, the gut concentration of orally administered DEX would readily activate intestinal PXR. Importantly, these results are directly relevant to the pediatric ALL patients treated with DEX as the plasma concentrations achieved with the oral discontinuous regimen in mice are similar to those achieved in patients treated with conventional DEX therapy (Ramsey et al., 2014).

We recently found in a murine BCR-ABL ALL model VD3 sufficient mice died earlier than $\mathrm{VD}_{3}$ deficient mice (Chapter 4). Given our finding that $\mathrm{VD}_{3}$ deficient mice had higher systemic DEX exposure compared to sufficient mice during the first days of discontinuous DEX treatment we might have expected DEX treatment to negate this VD3 deficient survival advantage. However, DEX treatment was unable to reverse the survival advantage of $\mathrm{VD}_{3}$ deficient over deficient mice with ALL. It remains to be determined whether the effects of $\mathrm{VD}_{3}$ on survival of mice from BCR-ABL ALL translate to humans.

The 5-year event free survival rates for pediatric ALL have almost reached $90 \%$ (Strahlendorf, 2018). However, it is always important to consider whether a therapeutic intervention -e.g., vitamin $\mathrm{D}_{3}$ to treat vitamin $\mathrm{D}$ deficiency, might cause an unexpected negative side-effect - in this case an unfavorable CYP3A vitamin D3-mediated drug interaction. Our results in mice suggest that vitamin D supplementation to vitamin D insufficient/deficient patients will not affect systemic exposures of DEX or dasatinib. Our results do suggest the intriguing possibility that whether a patient is $\mathrm{VD}_{3}$ sufficient versus deficient may have an impact on the magnitude of CYP3A-mediated drug interaction with CYP3A inducers (including DEX) for substrates that undergo significant first pass intestinal metabolism (e.g., atorvastatin) (Schwartz, 2009). 


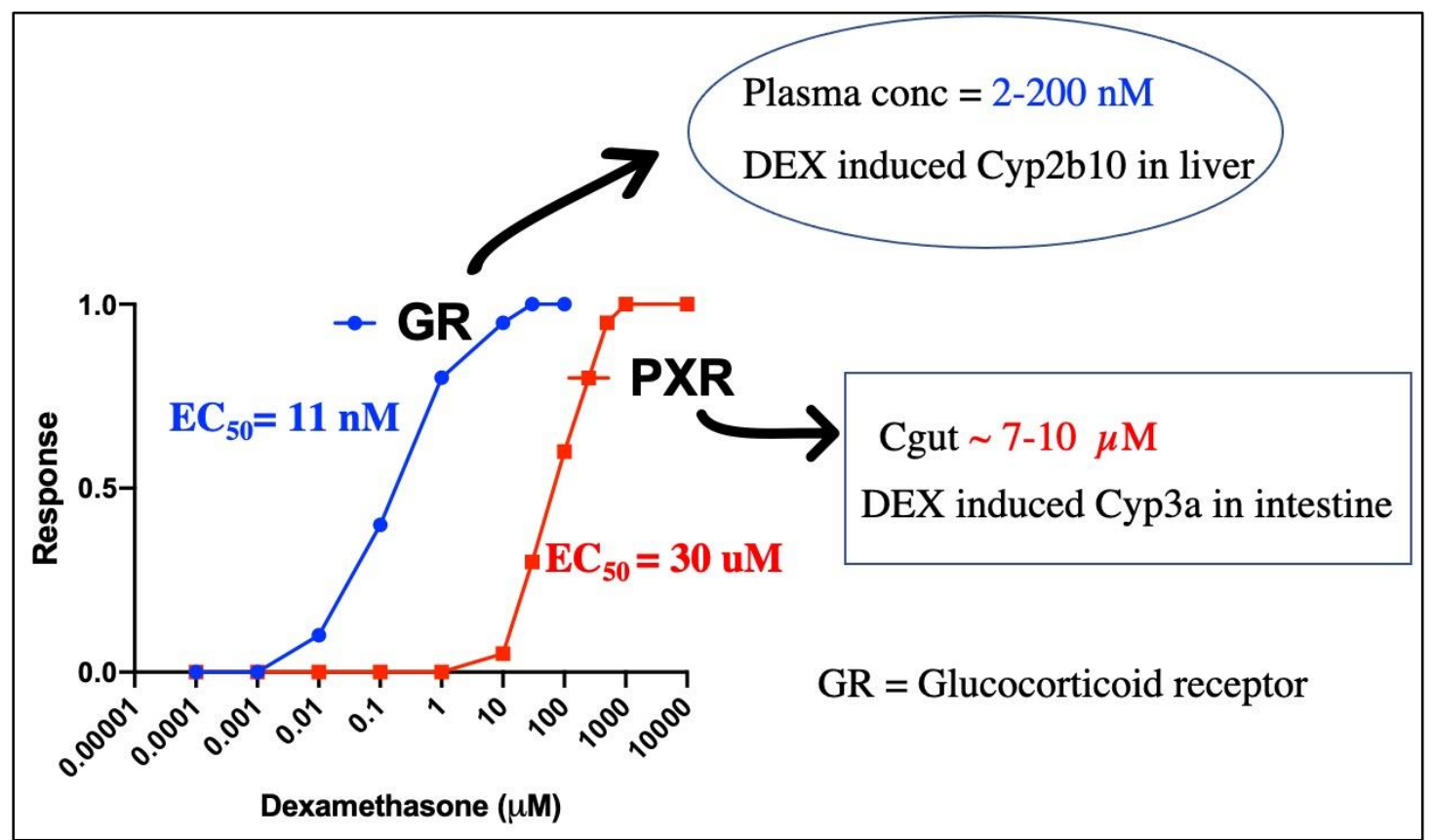

Figure 3-17. Differences in plasma and gut dexamethasone concentrations following oral discontinuous regimen leads to tissue specific activation of GR and PXR.

Data source: Kliewer, S. A., Moore, J. T., Wade, L., Staudinger, J. L., Watson, M. A., Jones, S. A., . . Lehmann, J. M. (1998). An orphan nuclear receptor 


\section{CHAPTER 4. ROLE OF VITAMIN D IN BCR-ABL ARF-/- ACUTE LYMPHOBLASTIC LEUKEMIA ${ }^{1}$}

\section{Introduction}

Vitamin D deficiency affects an estimated 1 billion people in the world across all ethnicities and age groups (Boscoe \& Schymura, 2006; Holick, 2007; Holick et al., 2011). VDD is an independent risk factor for mortality in the general population (Melamed, Michos, Post, \& Astor, 2008) and almost 60\% of children with malignant diseases have suboptimal vitamin D levels (Sinha, Avery, Turner, Bailey, \& Cheetham, 2011). VDD is not limited to developing countries, a recent study found that among 45 people tested in Memphis, TN for vitamin A and vitamin D levels, only two individuals had sufficient levels of both vitamins (B. G. Jones et al., 2016).

Vitamin D is a fat-soluble vitamin that not only regulates calcium absorption and bone metabolism, but can also regulate cell proliferation, differentiation and the immune response. The biologically active form of Vitamin $\mathrm{D}, 1,25(\mathrm{OH})_{2} \mathrm{VD}_{3}$, binds to the vitamin $\mathrm{D}$ receptor (VDR) that heterodimerizes with the retinoid $\mathrm{X}$ receptor (RXR), and that complex binds to VDR-RXR response elements in target genes to regulate transcription. VDR is highly expressed in intestine, kidney and bone, but also in normal and neoplastic hematopoietic cells and mesenchymal stem cells in bone marrow (Haussler et al., 1998). 1, 25-(OH) $2 \mathrm{VD}_{3}$, can modify embryonic hematopoietic stem and progenitor cell production (Cortes et al., 2016). 1, 25(OH) $2 \mathrm{VD}_{3}$ inhibits proliferation of mouse and human myeloid leukemia cells (Tanaka et al., 1982) and stimulates myeloid cell differentiation into mature macrophages. Indeed, mice with acute myeloid leukemia when treated with analogs of $1,25(\mathrm{OH})_{2} \mathrm{VD}_{3}$ survived longer than the untreated mice (Abe et al., 1981). Moreover, vitamin D insufficiency/deficiency is associated with poor clinical outcome and significantly worse progression free survival of patients from AML (H. J. Lee et al., 2014), chronic lymphoid leukemia (Shanafelt et al., 2011), and nonHodgkin lymphoma (Drake et al., 2010) indicating the clinical significance of vitamin D levels in patients with AML and CLL.

Acute lymphoblastic leukemia (ALL) is the most common form of childhood cancer accounting for almost $80 \%$ of pediatric leukemias (Madhusoodhan, Carroll, \& Bhatla, 2016), and multiple reports suggest that a majority of leukemia patients are $\mathrm{VD}_{3}$ deficient at the time of diagnosis (Naz, Qureshi, Shamsi, \& Mahboob, 2013; Sinha et al., 2011). Accordingly, both Dana Farber (Identifier number: NCT01574274) and Children's Hospital of LA (Identifier number: NCT01317940) have ongoing pediatric ALL clinical trials to monitor patients' $\mathrm{VD}_{3}$ levels and to give supplementation to restore sufficiency. However, it is not known how $\mathrm{VD}_{3}$ deficiency or supplementation might affect ALL

\footnotetext{
${ }^{1}$ Modified from final submission with open access permission. Annu, K., Cline, C., Yasuda, K., Ganguly, S., Pesch, A., Cooper, B., .. . Schuetz, E. G. (2020). Role of Vitamins A and D in BCR-ABL Arf(-/-) Acute Lymphoblastic Leukemia. Sci Rep, 10(1), 2359. doi:10.1038/s41598-020-59101-4 (*Co-first author) (Annu et al., 2020).
} 
progression or survival. Among the few reports of $\mathrm{VD}_{3}$ effects on the B-cell lineage of ALL (B-ALL) (the most common form of pediatric ALL), there have been conflicting results; one study concluded that $\mathrm{VD}_{3}$ had no effect on leukemia cell growth in suspension cultures (Antony et al., 2012), while another study concluded that $\mathrm{VD}_{3}$ inhibited B-cell ALL growth in a clonigenic soft agar assay (Consolini et al., 2001). Given the controversy in the literature, and the fact that a considerable number of ALL patients are $\mathrm{VD}_{3}$ deficient and some of them are receiving $\mathrm{VD}_{3}$ supplementation, it is important to test whether $\mathrm{VD}_{3}$ levels affect B-cell ALL cell growth, survival and prognosis.

B-ALL is classified into different sub-types based on a number of chromosomal abnormalities, and loss-of function or dominant-negative sequence mutations. Three-tofive percent of pediatric ALL cases and $25 \%$ of adult ALL cases, carry the translocation between chromosomes 9 and $22[\mathrm{t}(9 ; 22)]$ creating the BCR-ABL1 fusion gene (the Philadelphia chromosome $(\mathrm{Ph}+))($ Williams \& Sherr, 2008). At diagnosis around $67 \%$ of pediatric Ph+ ALL patients also have Ink4-Arf deletion (Arf-/-) (hereafter called BCRABL ALL or BCR-ABL+ ALL) and approximately $80 \%$ have IKAROS (IKZF1) alterations, which are considered hallmarks for high-risk B-ALL (Mullighan et al., 2008). Therefore, we have tested the effects of vitamin D sufficiency versus deficiency on survival in a murine BCR-ABL leukemia model and probed the mechanisms of their differential effects.

\section{Materials and Methods}

\section{Animals}

C57BL/6, day 4-5 estrus pregnant (E4-5) female mice were obtained from Jackson Laboratories (Bar Harbor, ME, USA). Animals arriving at the St. Jude Children's Research Hospital (SJCRH) animal facility were placed Purina Mills (Purina Mills, St. Louis, MO, USA) diets - either control (cat. no. 5W9M contained 1.5 IU of vitamin D), VDD (cat. no. 5W9X, contained 0 IU of vitamin D) were used for the survival and other in vivo studies.

Pregnant dams were maintained on these diets from the time of arrival, throughout the pregnancy, and after parturition. At weaning mouse pups were maintained on their respective diets. We also used a different strategy to generate vitamin D deficiency in mice by transitioning them to a VDD diet at weaning. This would eliminate any potential effect of a vitamin $\mathrm{D}$ deficient diet on the status of any other systems in pups derived from pregnant mom's put on VDD diets at pregnancy day 5. Hence, mice in a separate set of survival studies, were placed at weaning on a VDD diet (Harlan catalog no. 5A69, 0 IU of vitamin D) and vitamin D sufficient control diet (catalog. no. 5BV8, 3.3 IU of vitamin D). 
Serum 25-(OH) $\mathrm{VD}_{3}$ levels were routinely measured from representative mice in the VDD group (starting at 4 weeks of age) to determine vitamin D status. A level of 20$50 \mathrm{ng} / \mathrm{mL}$ is considered vitamin D sufficient; $12-20 \mathrm{ng} / \mathrm{mL}$ is vitamin D insufficient, and less than $12 \mathrm{ng} / \mathrm{mL}$ is vitamin D deficient. Sera were sent to Michigan State University (Lansing, MI, USA) for $25-(\mathrm{OH}) \mathrm{VD}_{3}$ testing. Serum $25-(\mathrm{OH}) \mathrm{VD}_{3}$ levels were also measured in mice at the terminal time point in the leukemia survival studies.

\section{Ethical Approval Statement}

Association for Assessment and Accreditation for Laboratory Animal Care (AALAC) guidelines and Institutional Animal Care and Use Committee (IACUC) approved protocols were followed for all the experimental procedures and housing of the mice. All methods were carried out in accordance with the relevant guidelines and regulations.

\section{Leukemia and Bone Marrow Stroma Cells}

Murine BCR-ABL+ Arf-/- cells tagged with luciferase (Luc+) or green fluorescent protein (GFP+) (Williams, Roussel, \& Sherr, 2006) created by Dr. Charles Sherr's lab and provided by Dr. Mary Relling's lab, were cultured as previously described (Ramsey et al., 2015). Human mesenchymal stromal cells (hMSCs, bone marrow stroma) immortalized with telomerase (Mihara et al., 2003) and created by Dr. Dario Campana's lab, were provided by Dr. Jun Yang's lab, and cultured as described (Iwamoto, Mihara, Downing, Pui, \& Campana, 2007).

\section{BCR-ABL ALL Disease Generation and Monitoring}

On day zero, 2,000 BCR-ABL Arf-/- Luc+ cells were injected retro-orbitally into syngeneic, unconditioned 6-10-week-old mice, alternating injection order between cages and groups. The first experiment was run with control male (M) (n=17), VDD M (n=23) and control female $(\mathrm{F})(\mathrm{n}=14)$, VDD F $(\mathrm{n}=14)$ mice. Mice were anesthetized with isoflurane and images using Xenogen IVIS-200 (Caliper Life Sciences, Hopkinton, MA) were acquired post-intraperitoneal injection of $200 \mu \mathrm{L}$ of $100 \mathrm{mg} / \mathrm{kg}$ D-Luciferin (Caliper Life Sciences, Hopkinton, MA). The bioluminescent signal (photons/s) was acquired from a fixed region of interest (ROI) starting on day 8, repeating every 3-4 days to monitor disease progression (Figure 4-1). Acquired images were analyzed with Living Image 3.1 software (Caliper Life Sciences). BCR-ABL-Luciferase disease burden was quantified from whole animal total luciferase flux measurements (photons/s) from the images.

Moribund state of mice was determined based on hind limb paralysis, a scruffy coat, lethargy, or an inability to obtain food or water. At this time before euthanasia, blood was collected for the analysis of cholesterol, triglycerides, high-density lipoprotein 


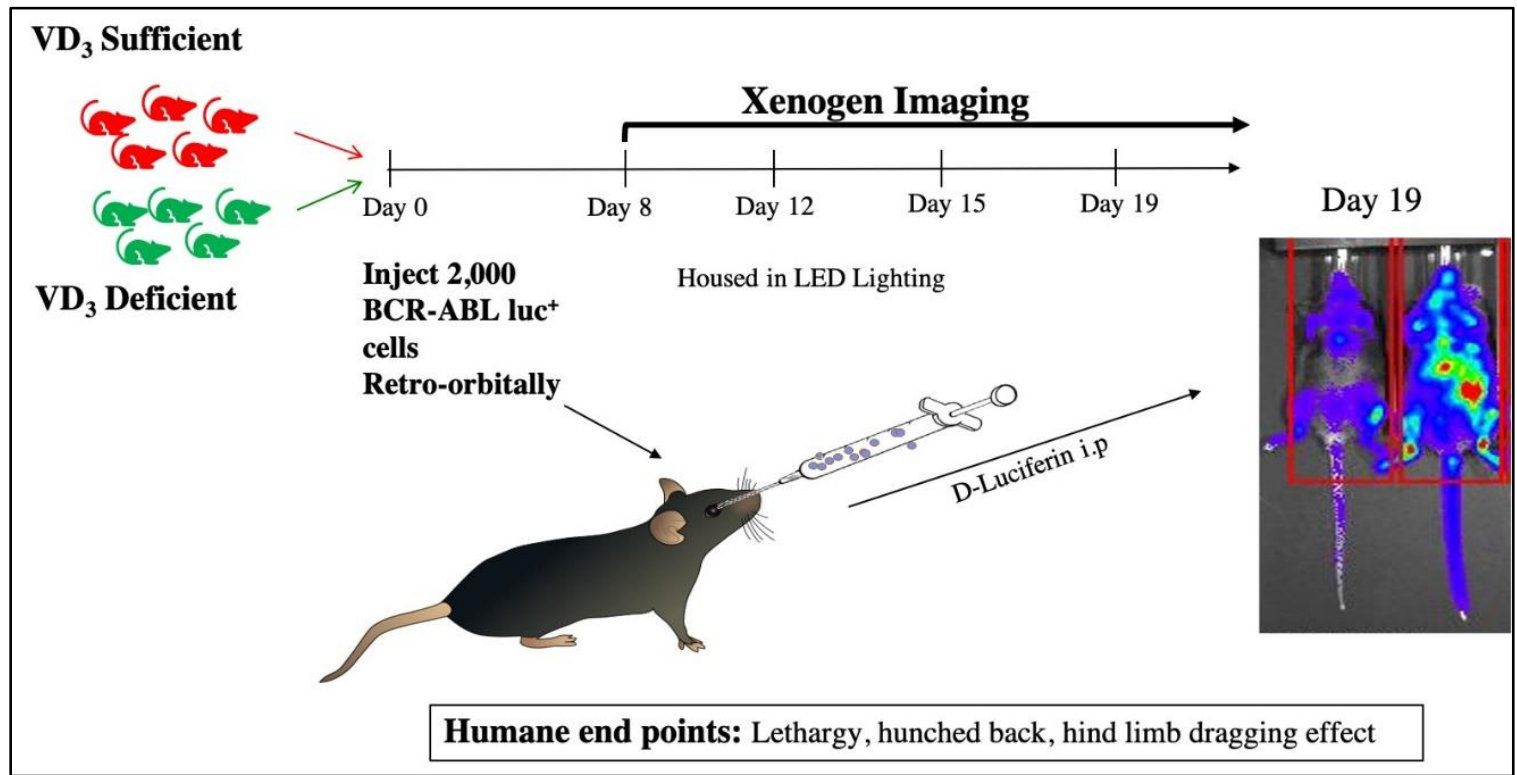

Figure 4-1. BCR-ABL ALL disease generation and monitoring in mice. 
(HDL) and low-density lipoprotein (LDL), white blood cell number, and serum for 25$(\mathrm{OH}) \mathrm{VD}_{3}$. Spleen was harvested and bone marrow cells from femur were flushed using a needle with either cell culture media or RNAlater (Invitrogen by ThermoFischer Scientific, Vilnius, LT). The cells were transferred to a $1.5 \mathrm{~mL}$ eppendorf tube and pelleted, the cell pellets were resuspended in $100 \mu \mathrm{L}$ PBS, and transferred to white, clearbottomed 96-well plate (Costar, Corning Inc., Kennebunk, ME, USA) to which $100 \mu \mathrm{L}$ of Bright-Glo ${ }^{\mathrm{TM}}$ (Promega, Madison, WI, USA) was added. A standard curve with a range of 3,000 to 2 million BCR-ABL cells was used in order to convert the luminescence value into the number of BCR-ABL cells per well. Luminescence was measured using the BioTek Synergy ${ }^{\mathrm{TM}} 4$ Hybrid Microplate Reader.

A portion of spleen, hind limb was individually fixed in $10 \%$ formalin; 24 hours later the tissues were transferred to Cal-Rite (Thermo Scientific, Pittsburgh, PA) for a minimum of 48 hours. Tissues were embedded in paraffin, and $4 \mu \mathrm{m}$ sections were cut and stained with hematoxylin and eosin. Slides were evaluated by a pathologist to determine the extent of leukemia burden, scoring the tissues on a 6- point scale from 0-5. The entire experiment was repeated, and both studies were combined for the data analysis with a total of control M ( $\mathrm{n}=34)$, VDD M $(\mathrm{n}=34)$ and control F (n=27), VDD F ( $\mathrm{n}=25)$ mice.

To measure bone marrow vascularity and trabecular volume, the slides were scanned using an Aperio ScanScope (Aperio Technologies, Vista, CA) with a 20X objective. Measurements were performed using ImageScope software. The metaphyseal region defined as beginning at the physis and ending $1 \mathrm{~mm}$ distal to it; this marrow area was outlined to obtain total metapyseal area. For measurement of bone marrow vascularity all vascular lumens (sinusoids) were outlined within that area. Two types of data calculated from these measurements, \% vascular area (um2 vessel lumen/ um2 marrow), and length (um vessel/ um2 marrow). For trabecular volume, all trabeculae were outlined within this area and the output was calculated as \% trabecular area (um2 trabecular bone/ um2 marrow). Osteoblast and osteoclast numbers, as well as leukemia burden, were evaluated semi-quantitatively by a veterinary pathologist (L.J.J.) using a 5point scale.

\section{PCR Assays}

\section{PCR to determine gender of BCR-ABL ALL cells}

The gender of the murine BCR-ABL+ Arf-/- cells was determined by PCR of genomic DNA for the presence of $\mathrm{X}$ and $\mathrm{Y}$-chromosome specific markers (McFarlane, Truong, Palmer, \& Wilhelm, 2013). DNA was amplified with the following primer pairs:

Sly/Xlr_F, 5'-GATGATTTGAGTGGAAATGTGAGGTA-3'; Sly/Xlr-R, 5'-CTTATGTTTATAGGCATGCACCATGTA-3' and Zfy_F, 5'-GACT A GACATGTCTTAACATCTGTCC-3' and 
Zfy_R, 5'-CC T A TTGCATGGACTGCAGCTTATG-3' and amplification conditions were as described (McFarlane et al., 2013).

\section{PCR to detect VDR mRNA}

The expression of VDR in BCR-ABL+ Arf-/- and LS180 cells was determined by PCR. DNA was amplified using the following primer pairs:

mVDR-F, CTCCTCGATGCCCACCACAAGACCTACG; mVDR-R, GTGGGGCAGCATGGAGAGCGGAGACAG; hVDR-F, CGGCCGGACCAGAAGCCTTT; hVDR-R, CGGGGCACGTTCCGGTCAAA; GAPDH-F, ACCACAGTCCATGCCATCAC; GAPDH-R, TCCACCACCCTGTTGCTGTA.

Promega PCR master mix (catalog number M7505), and amplification conditions are as described below: Denaturation at $95^{\circ} \mathrm{C}$ for $2 \mathrm{~min}$; 34 cycles (mVDR) or 40 cycles (hVDR): denaturation at $95^{\circ} \mathrm{C}$ for $30 \mathrm{sec}$, annealing at $60^{\circ} \mathrm{C}$ for $30 \mathrm{sec}$ and extension at $72^{\circ} \mathrm{C}$ for $30 \mathrm{sec}$; extension at $72^{\circ} \mathrm{C}$ for $5 \mathrm{~min} ; 4^{\circ} \mathrm{C}$ hold. The product was mixed with $6 \mathrm{x}$ DNA loading dye (Promega, WI, USA) and ran on a $2 \%$ agarose gel (used SYBR Safe DNA gel stain) at $100 \mathrm{~V}$ for 90 minutes. Images were taken using Image Lab SoftwareTM.

\section{Analysis of in vivo Growth of Leukemic Burden}

Change in leukemia burden with time was analyzed from the change in whole body luminescence data. Luminescence data for every mouse was normalized with its baseline luminescence value from day 8 . Fold change in luminescence over baseline was plotted against days, which was assumed to follow the exponential growth equation, Fold change in luminescence $=A \mathrm{e}^{\wedge}\left(\mathrm{B}^{*} \mathrm{t}\right)$, where, $\mathrm{A}$ is the intercept and $\mathrm{B}$ is the slope of the exponential growth curve. Parameters A and B were estimated by fitting the fold change in luminescence versus days for each mouse using Microsoft Excel-based trendline analysis. Correlations were considered acceptable only if $r^{2}>0.7$. All the survivors were removed from the analysis, as we do not expect to have increase/growth in leukemia burden in these mice. Other filtration criteria were: 1 ) fold change in luminescence has to be $>1$, i.e., increase in luminescence over the baseline, 2) positive correlation of foldchange in luminescence with days, 3 ) there have to be at least three data points for the assessment of correlation, and 4) since the same number of leukemic cells were injected into the mouse, we expected to have no significant difference in intercept $\mathrm{A}$ and thus outlier intercepts were removed from the analysis. After filtration of the data, individual slopes (B) were computed for each mouse and compared between the groups, data represented as median slope \pm range and Mann-Whitney test at $p<0.05$ was used to analyze the significance. 


\section{BCR-ABL ALL Viable Cell Number Assay}

Luciferase-tagged BCR-ABL cells were plated at a density of 500 cells per well on a 24 well plate and treated with either $1,25-(\mathrm{OH})_{2} \mathrm{VD}_{3}(50$ or $100 \mathrm{nM})$ or ethanol (vehicle) and the plates incubated at $37^{\circ} \mathrm{C} 8 \% \mathrm{CO}$. To select the concentration of 1,25$(\mathrm{OH})_{2} \mathrm{VD}_{3}$ in cell culture, we first measured dose response effect on BCR-ABL ALL viable cell number and found no effect with 1-10 nM, but equal effectiveness of 50-100 $\mathrm{nM}$. Since the duration of our $1,25(\mathrm{OH})_{2} \mathrm{VD}_{3}$ studies was routinely $48 \mathrm{hr}$ (but as long as $96 \mathrm{hr}$ ), and the vitamin is not replenished after dosing at zero hr, and 1, 25-(OH $)_{2} \mathrm{VD}_{3}$ has a half-life of $15 \mathrm{hr}$, the effective concentration remaining at the end of a $48 \mathrm{hr}$ experiment following a $100 \mathrm{nM}$ treatment is $25 \mathrm{nM}$.

In humans the bone marrow concentration of $1,25-(\mathrm{OH})_{2} \mathrm{VD}_{3}$ is 500 -fold higher than the bone marrow plasma concentration (Blazsek et al., 1996). Since the plasma concentration of $1,25(\mathrm{OH})_{2} \mathrm{VD}_{3}$ is $30 \mathrm{pg} / \mathrm{ml}(0.072 \mathrm{nM})$, the bone marrow stroma concentration at 500-times that $=36 \mathrm{nM}$. Hence, $50-100 \mathrm{nM} 1,25(\mathrm{OH})_{2} \mathrm{VD}_{3}$ was used. To measure cell number, at the terminal time point for vehicle, $1,25(\mathrm{OH})_{2} \mathrm{VD}_{3}$ treated wells, the entire contents of each well were transferred to a $1.5 \mathrm{~mL}$ eppendorf tube, the cells were pelleted, resuspended in $100 \mu \mathrm{L} \mathrm{PBS}$, and transferred to white clear-bottomed 96-well plates (Costar Corning Inc., Kennebunk, ME, USA) and $100 \mu \mathrm{L}$ of Bright-Glo ${ }^{\mathrm{TM}}$ (Promega, Madison, WI, USA) was added. Luminescence was read using the BioTek Synergy ${ }^{\text {TM }} 4$ Hybrid Microplate Reader. Standard curves were prepared in triplicate using known numbers of Luc+ BCR-ABL cells, then the number of BCR-ABL cells in experimental samples was determined by interpolation, and results plotted of average cell number/treatment group or fold differences between vitamin treated and vehicle-treated wells. Hence, the assay measures the difference in total number of cells between treatment groups at a single time point. The assay measures number of viable (not dead cells) because the assay depends on the number of cells that are viable and metabolically active.

\section{In vitro Homing Assay}

hMSC (100,000/well) were plated onto the bottom of 24-well plates and treated with ethanol vehicle or $100 \mathrm{nM} 1,25(\mathrm{OH})_{2} \mathrm{VD}_{3}$ for 48 hours with half of the wells/group treated with media lacking fetal bovine serum (FBS). Identical 24-well plates without hMSC were prepared. Then $6.5 \mathrm{~mm}$ Transwell Permeable Supports with a $3 \mu \mathrm{m}$ polycarbonate membrane (Costar Corning Inc., Kennebunk, ME, USA) were added to each well and 1 million BCR-ABL luciferase(luc)+ cells in $100 \mu \mathrm{L}$ of media was added to top donor compartment and incubated at $8 \% \mathrm{CO} 2$ for $24 \mathrm{hr}$. The bottom of each transwell membrane (Figure 4-2) was then washed with media to collect any attached cells. A cell scraper was used to remove all cells from the wells, which were pelleted and resuspended in $100 \mu \mathrm{L}$ PBS and $100 \mu \mathrm{L}$ of Bright-Glo ${ }^{\mathrm{TM}}$ (Promega, Madison, WI).

The BioTek Synergy ${ }^{\mathrm{TM}} 4$ Hybrid Microplate Reader was used to read the luminescence on a white, clear-bottom 96 well plate (Costar, Corning Incorporated, 


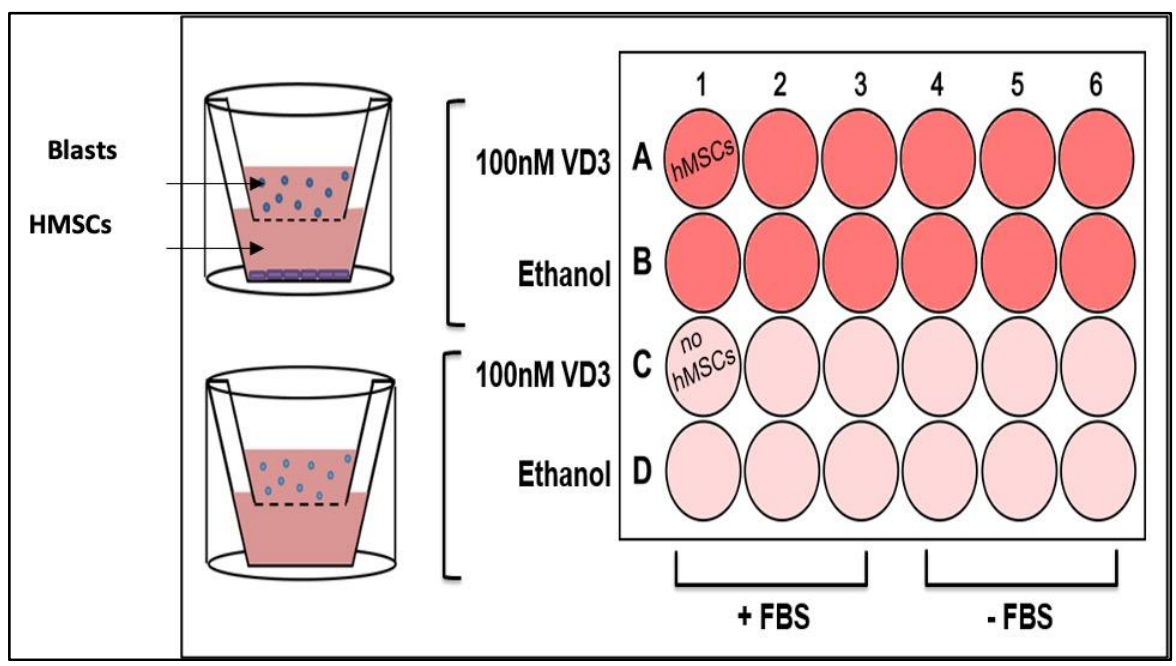

Figure 4-2. BCR-ABL cell migration in vitro using transwell membrane. 
Kennebunk, ME, USA). Luminescence from 3,000 - 2 million BCR-ABL luc+ cells was used to generate a standard curve and the relative BCR-ABL cell number in the receiver compartment was interpolated from the standard curve which was used to determine the percentage of total cells that had migrated from the donor to the receiver compartment.

\section{Real-Time PCR}

A confluent layer of human bone marrow mesenchymal stem cells (hMSCs) immortalized with telomerase (Mihara et al., 2003) were treated with vehicle or 100nM $1,25(\mathrm{OH})_{2} \mathrm{VD}_{3}$ for 48 or 72 hours. RNA was extracted using Trizol (Ambion Life technologies, CA, USA or Thermo Fisher Scientific, CA, USA) and the Qiagen RNEasy clean-up kit (Hilden, Germany). 500ng of RNA was used to create cDNA using the ThermoScript ${ }^{\mathrm{TM}}$ RT-PCR System (Invitrogen ThermoFischer, CA, USA) that was then diluted to a total volume of $60 \mu \mathrm{L}$. Primers (Invitrogen, Carlsbad, CA) for amplification of human CXCL12/SDF-1 $\alpha$ were (F) 5'-AGAACTGTTGGCAAGGTGACA-3' and (R) 5'-CTGACATTCATATGGCTCTCATTC-3'; and for mouse CXCL12/ SDF-1 $\alpha$ were (F) 5'-CAGTGACGGTAAACCAGTCAGC- 3' and (R) 5'-TGGCGATGTGGCTCTCG- 3'. Samples were run at $95^{\circ}$ for 15 minutes and then 40 cycles of $92^{\circ}$ for 30 seconds, $60^{\circ}$ for 30 seconds, and $68^{\circ}$ for 1 minute followed by a dissociation step for the real-time PCR.

\section{CXCL12 ELISA Assay}

The Quantikine ELISA Kit (R\&D Systems, Minneapolis, MN, USA) for human CXCL12/SDF-1 $\alpha$ was used to assay both cell culture media and mouse serum according to the manufacturer's protocol. A standard curve was generated using the included controls and used for comparison to a positive control as well as the unknown samples. Cell culture media samples $(n=3)$ from $h M S C s$ in a 6-well dish were assayed undiluted after 48-hour treatment with either ethanol or $100 \mathrm{nM} 1,25(\mathrm{OH})_{2} \mathrm{VD}_{3}$. Undiluted serums from mice on either a $\mathrm{VD}_{3}$ sufficient (control) or $\mathrm{VD}_{3}$ deficient diet (VDD group; $\mathrm{n}=5$ per group) were also assayed using this approach. The BioTek Synergy ${ }^{\mathrm{TM}} 4$ Hybrid Microplate Reader was used to measure fluorescence at $450 \mathrm{~nm}$ and $540 \mathrm{~nm}$. Final values used for comparison were determined by subtracting the reading at $540 \mathrm{~nm}$ from the reading at $450 \mathrm{~nm}$; this step was designed to correct for optical imperfections in the plate.

\section{Immunohistochemistry}

VDD and control mice were perfused with $4 \%$ paraformaldehyde and the hind limbs were fixed in $10 \%$ neutral buffered formalin, decalcified, embedded in paraffin, sectioned $(4 \mu \mathrm{m})$ and placed onto glass slides for staining. Antibodies for IHC were obtained from Abcam and diluted in Phosphate Buffer Saline with 0.3\% Triton X-100 (PBST). Slides containing either mouse tibia or mouse hind limb were deparaffinized and antigens were retrieved using Target Retrieval solution pH 6.0 (Dako, Carpinteria CA, USA) in a pressure cooker for 15 min. After retrieval, slides were rinsed with PBST, 
treated with 3\% hydrogen peroxide, and blocked with Background Sniper (Biocare Medical, Pacheco, CA, USA). Slides were incubated overnight at $4^{\circ} \mathrm{C}$ with primary specific antibodies (rabbit anti-collagen I, ab34710 (1:500) and rabbit anti-collagen V (ab7046) (1:50), or the appropriate isotype control antibody, rinsed twice with PBST, and incubated with the secondary antibody (Donkey anti-rabbit 568, ab10042) for $2 \mathrm{hr}$. Slides were rinsed again in PBST and mounted using ProLong® Gold Antifade Mountant with DAPI (Molecular Probes by Life Technologies ${ }^{\mathrm{TM}}$, Eugene, OR, USA) and allowed to dry overnight before viewing. Images were obtained (60X magnification) using the Marianas Microscopy System (Intelligent Imaging Innovations).

\section{Flow Cytometry}

Age matched $\mathrm{VD}_{3}$ Sufficient and deficient mice are used for this assay. Isolation of bone cells was performed as described in (Houlihan et al., 2012) with minor modifications to the protocol. Cardiac puncture was performed to harvest blood which is processed into serum and stored at $-80 \mathrm{C}$. This serum is used to measure the $25-(\mathrm{OH}) \mathrm{VD}_{3}$ levels in the mice. After euthanizing the mouse, both tibia and femur were dissected by remove any adherent muscle tissue and cleaned carefully using Kimwipe to remove any nonadherent tissue (Figure 4-3). Using a syringe filled with cold PBS, bone marrow is flushed into a different test tube. The bones are placed in $30 \mathrm{~mL}$ of ice-cold PBS. The bones were washed 3 times in fresh PBS by vigorous shaking. Using a sterile mortar and pestle, the bones were crushed with one fracture per bone and using scissors to cut the bone into small fragments. The bone fragments were washed 2-3 times using freshly made $10 \mathrm{~mL}$ of HBSS + solution. HBSS+ is made using Hank's balanced salt solution (HBSS) (Invitrogen, cat. no 14170112) as described in (Houlihan et al., 2012). Then the bone fragments are placed into $50 \mathrm{~mL}$ conical tubes filled with $20 \mathrm{~mL}$ of preheated DMEM with $0.2 \%$ collagenase and placed on a temperature regulated shaker at $37^{\circ} \mathrm{C}$ for 1 hour at 110 r.p.m. Then, the solution is filtered using a $70 \mu \mathrm{m}$ cell strainer and placed on ice. The fragments are placed into the mortar with HBSS+ solution and using pestle gentle tapping is performed to liberate the bone cells into the solution. After performing this step at least 6-7 times to obtain good yield, the suspension is centrifuged in a precooled centrifuge at $280 \mathrm{~g}$ for $10 \mathrm{~min}$ at $4^{\circ} \mathrm{C}$. Supernatant was discarded carefully and for red blood lysis to occur, the pellet was resuspended in $1 \mathrm{~mL}$ of ice-cold water for 6 sec. Immediately $1 \mathrm{~mL}$ of $2 x P B S$ with $4 \%$ (v/v) FBS was added to stop the lysis and the volume was made up to $15 \mathrm{~mL}$ using HBSS+. The solution is filtered through $70 \mu \mathrm{m}$ cell strainer and spined once again for 5 minutes at $280 \mathrm{~g}$ in $4^{\circ} \mathrm{C}$. Supernatant was discarded carefully, and the pellet was resuspended in $1 \mathrm{~mL}$ of HBSS+. This was later used for staining of the bone cells. The antibodies used, concentrations and isotypes used to stain the cells are shown below in the Table 4-1.

\section{Data Analysis}

Kaplan Meier curves were generated to compare survival data between the groups and the Gehan-Breslow-Wilcoxon Test was used to determine significance. The Welch's 


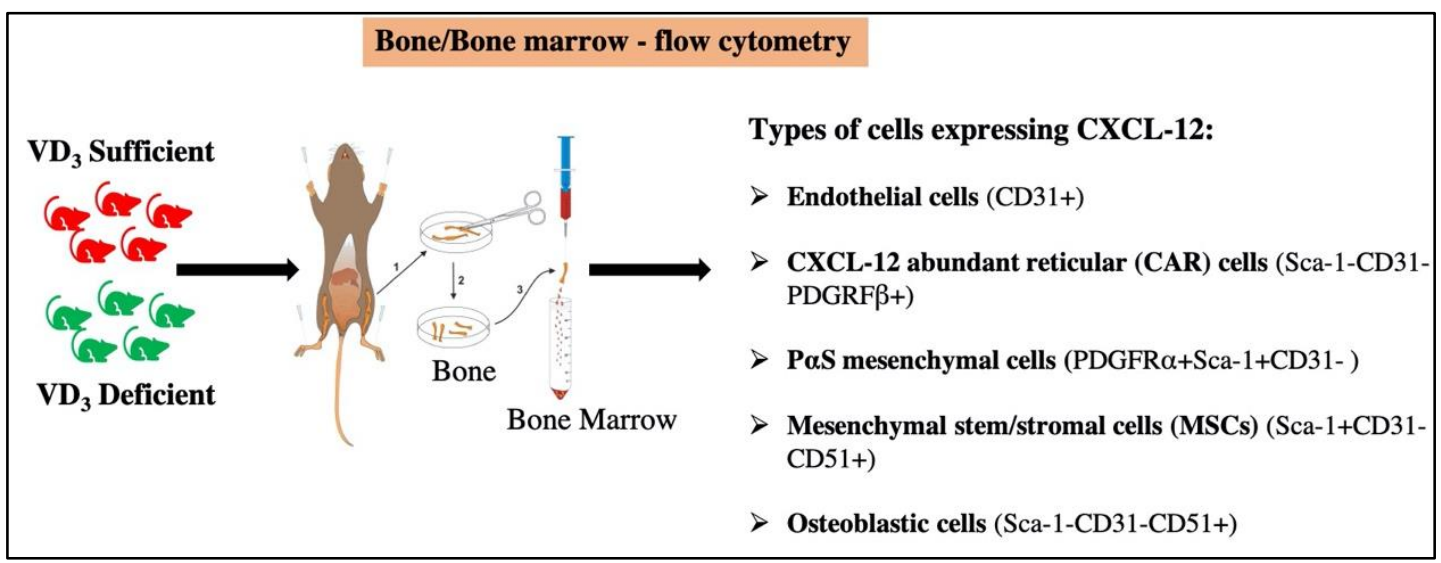

Figure 4-3. Experimental design - bone/bone marrow cells isolation and staining

Table 4-1. Antibodies used to stain bone and bone marrow cells for flow cytometry.

\begin{tabular}{llcl}
\hline $\begin{array}{l}\text { Protein and } \\
\text { fluorochrome }\end{array}$ & Company, Cat. no. & Concentration & \multicolumn{1}{c}{ Isotype } \\
\hline CD31, FITC & Biolegend, 102405 & $0.5 \mathrm{mg} / \mathrm{mL}$ & Rat IgG2a, K \\
CD51, PE & Biolegend, 104105 & $0.2 \mathrm{mg} / \mathrm{mL}$ & Rat IgG1, K \\
Sca-1, APC-Cy7 & Biolegend, 108125 & $0.2 \mathrm{mg} / \mathrm{mL}$ & Rat IgG2a, K \\
CD140a, PE-Cy7 & Biolegend, 135911 & $0.2 \mathrm{mg} / \mathrm{mL}$ & Rat IgG2a, K \\
CD140b, APC & ThermoFisher & $0.2 \mathrm{mg} / \mathrm{mL}$ & Rat IgG2a, K \\
& Scientific, 17-1402-82 & & \\
CD45, BV605 & Biolegend, 103139 & $0.2 \mathrm{mg} / \mathrm{mL}$ & Rat IgG2a, K \\
Ter119, BV711 & Biolegend, 116233 & $0.2 \mathrm{mg} / \mathrm{mL}$ & Rat IgG2b, K \\
CD71, BV786 & BD Biosciences, & & Rat IgG2b, K \\
\hline
\end{tabular}


t-test was used to determine significant differences in ROI means between the groups. Luciferase signals of blood, spleen, and bone marrow, and ELISA data were analyzed using Mann-Whitney nonparametric test on GraphPad Prism versions 5 and 7. Blood chemistry data were analyzed using 1way ANOVA with Tukey's multiple comparison test. Significance is calculated at $\mathrm{p}<0.05$ and outliers were determined using Grubb's test on GraphPad QuickCalcs. Real-Time PCR data was analyzed using the $\Delta \Delta C$ t method. Unpaired t-tests were used to determine significance in other assay's comparing cells treated with $1,25(\mathrm{OH})_{2} \mathrm{VD}_{3}$ or vehicle. Flow cytometry comparisons were done using unpaired t-tests using GraphPad Prism software.

\section{Results}

\section{Vitamin D Deficiency Improves Survival of Mice with BCR-ABL Arf-/- B-ALL}

Progression of BCR-ABL Arf-/- luciferase tagged pre-B leukemia cells and survival from disease were compared between vitamin $\mathrm{D}$ sufficient control mice and VDD mice by Xenogen imaging of leukemic cells starting on day 8 in male and female mice (Figures 4-4, 4-5). Leukemia was detected at day 8 in control male mice but was not detected in the VDD male mice until day 10. Surprisingly, compared to VD3 sufficient (control) mice, VDD mice showed a slower tumor progression over time and had lower average disease burden per mouse (Figure 4-6). Consequently, VDD mice survived significantly longer (Figure 4-7, $* *=0.003$ ) than $\mathrm{VD}_{3}$ sufficient control mice. This can also readily be seen by comparing the proportion of mice surviving on each day after leukemia was administered (Table 4-2). For example, study 1 on Day 18 only 47\% of the control male mice versus $80.95 \%$ of the VDD male mice were surviving, hence the median survival for the control male mice was 18 days versus 21 days for the VDD male mice. Female mice inoculated with tumor cells had slow growth of disease and longer median survival than males, so we determined the sex of the murine BCR-ABL leukemia cells using a PCR assay to discriminate $\mathrm{X}$ and $\mathrm{Y}$ chromosome specific genes in the leukemia cells. This PCR assay relies on Y chromosome specific Zfy amplification in males, but is absent in females, and amplification of a $280 \mathrm{bp}$ Y chromosome specific Sly gene product in males, and a 480/685 bp X chromosome specific Xlr gene product in females (McFarlane et al., 2013). The BCR-ABL cells were male as indicated by the Zfy and Sly Y chromosomal PCR products (Figure 4-8).

Vitamin D deficiency was confirmed in serum obtained from terminal blood samples by analysis of 25-(OH) $\mathrm{VD}_{3}$ in the control, VDD mice (Table 4-3). Complete blood count (CBC) (Table 4-4) and serum chemistries (Table 4-5) were compared between control, VDD mice with BCR-ABL ALL diseased and non-leukemic healthy mice (no disease controls (ND)), Compared to leukemic control mice, leukemic male mice deficient for vitamin D had decreased red blood cell number, hematocrit and hemoglobin consistent with higher leukemic burden in these mice. Comparing serum chemistries between the groups, the vitamin deficient males had elevated levels of HDL 


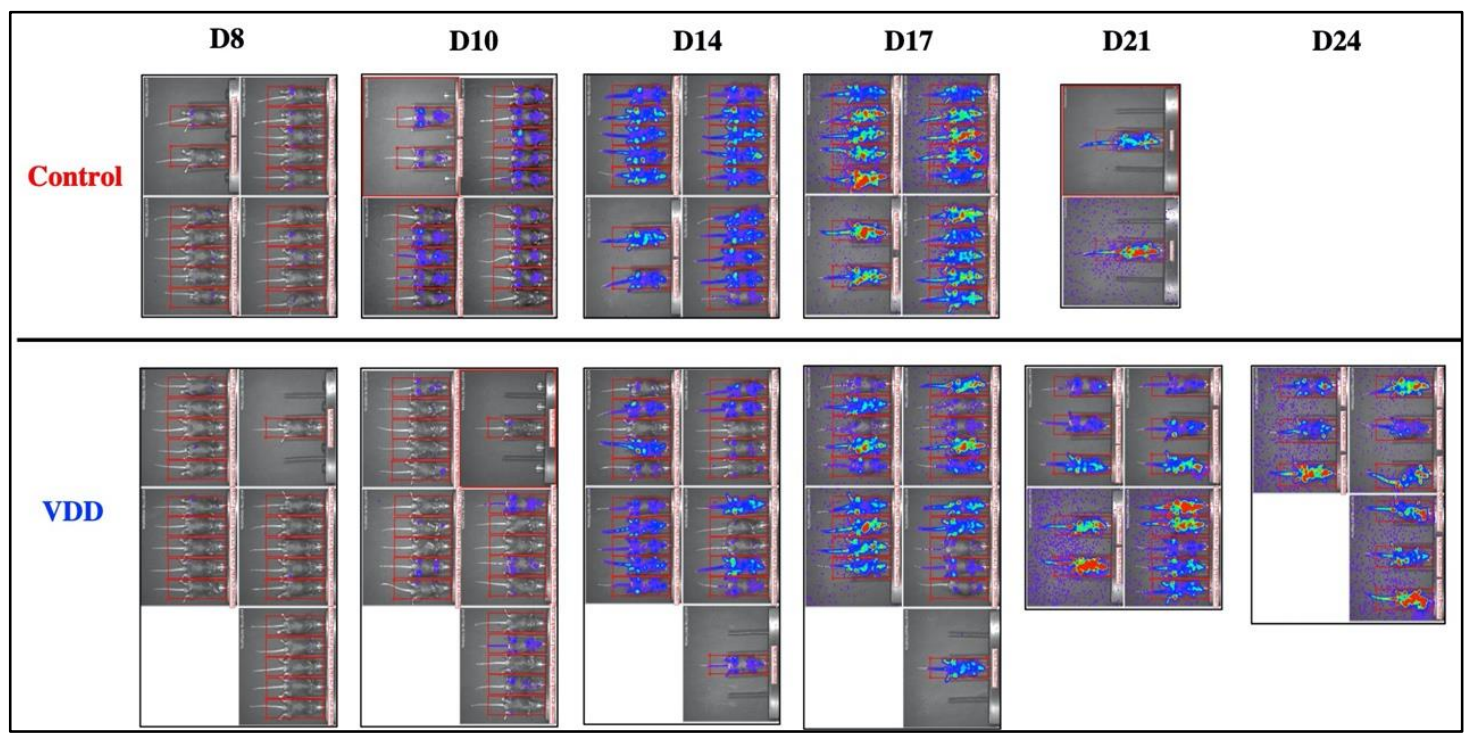

Figure 4-4. BCR-ABL luc+ ALL disease burden in male mice.

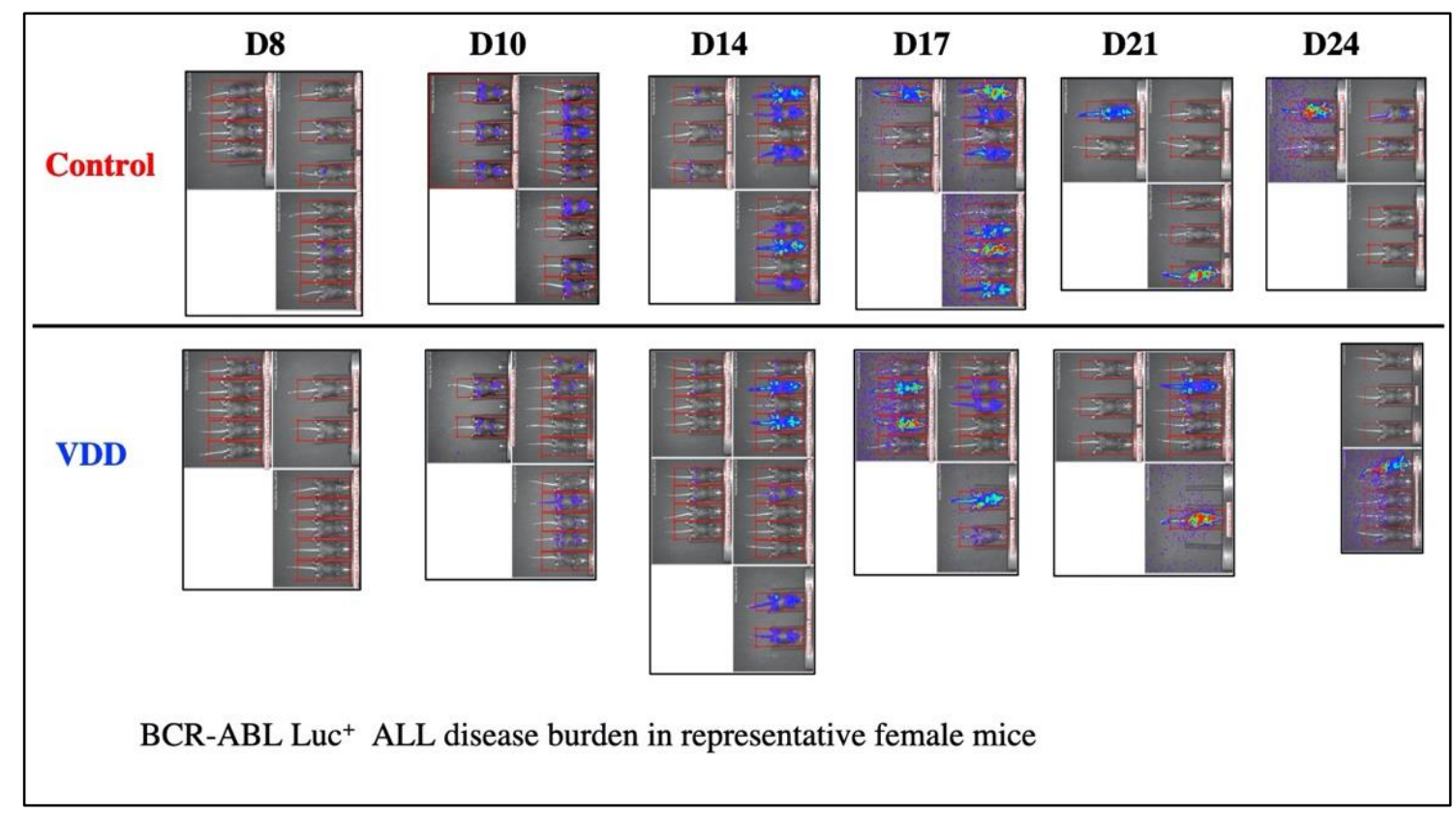

Figure 4-5. BCR-ABL luc+ ALL disease burden in female mice. 


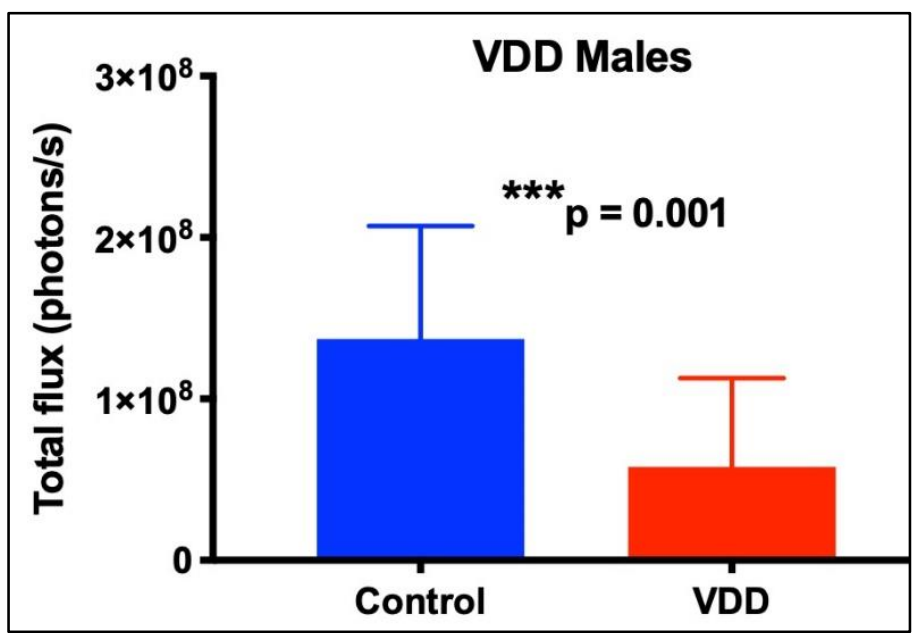

Figure 4-6. BCR-ABL ALL disease burden on day 17 in male control and VDD mice.

Notes: The unpaired t-test with Welch's correction was used to compare leukemia body burden $(* * * \mathrm{p}=0.001)$

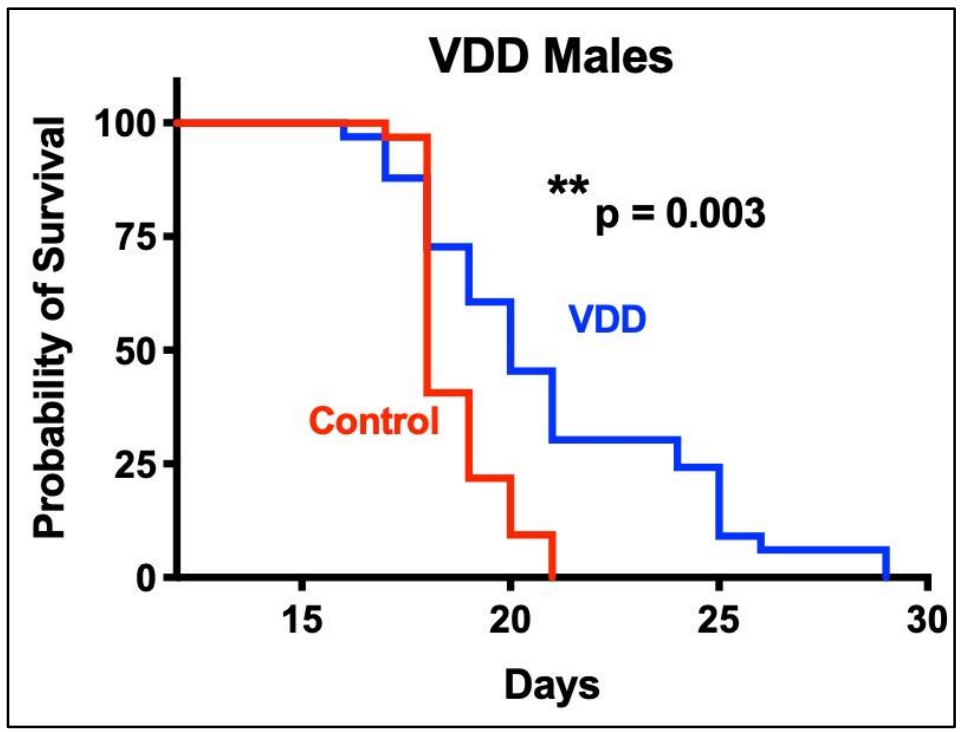

Figure 4-7. Kaplan Meier survival curve of male $\mathrm{VD}_{3}$ sufficient (control) and $\mathrm{VD}_{3}$ deficient mice.

Notes: The Gehan-Breslow-Wilcoxon test was used to find differences between survival curves of control and each vitamin deficient group $\left(* * * * \mathrm{p}<0.000,{ }^{* *} \mathrm{p}<0.01\right)$ 
Table 4-2. Survival proportions of control and vitamin D-deficient mice.

\begin{tabular}{ccc}
\hline Days elapsed & Control & $\begin{array}{c}\text { Vitamin D } \\
\text { deficient }\end{array}$ \\
\hline 0 & 100 & 100 \\
5 & & \\
16 & & 95.24 \\
17 & & 85.71 \\
18 & 47.06 & 80.95 \\
19 & 11.76 & 71.43 \\
20 & & 61.90 \\
21 & 0.00 & 42.86 \\
24 & & 33.33 \\
25 & & 9.52 \\
29 & & 0.00 \\
\hline
\end{tabular}

Notes: Control $=$ Vitamin D sufficient

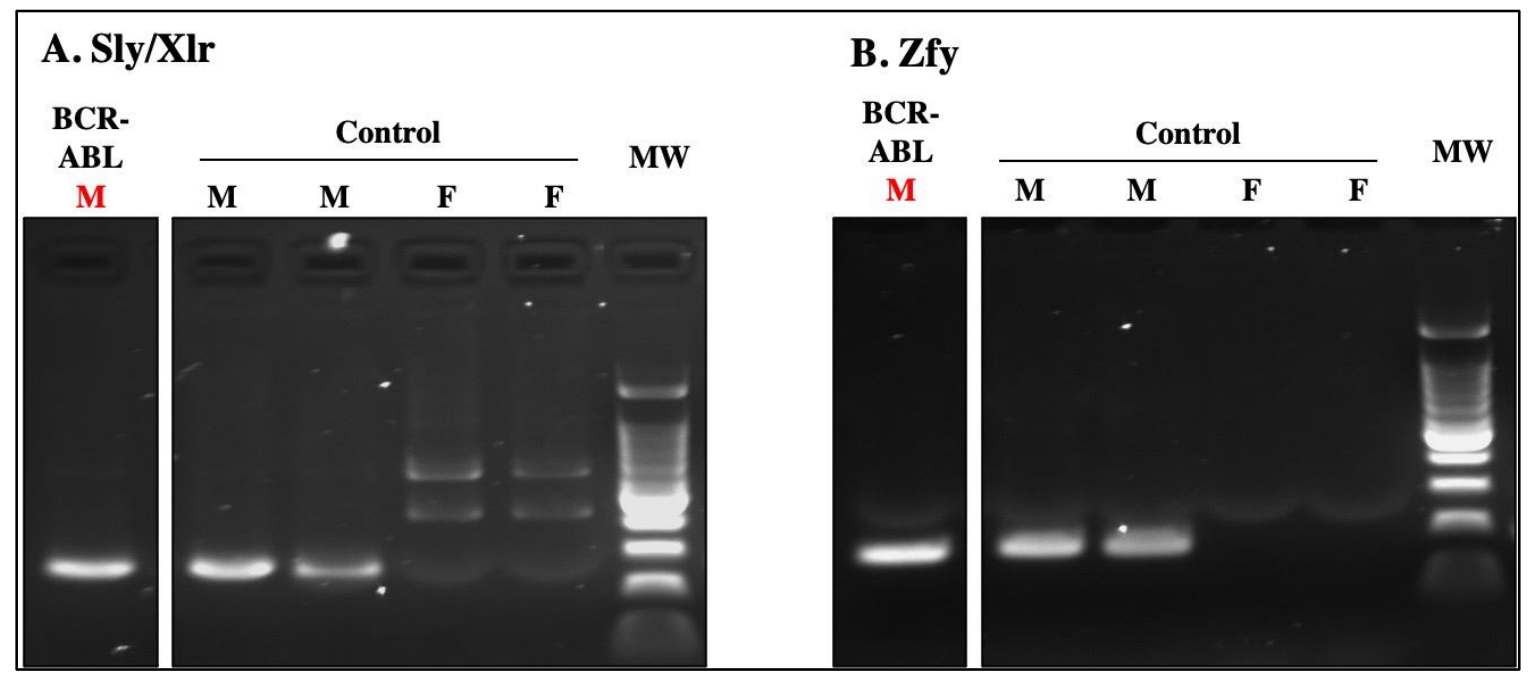

Figure 4-8. Determination of mouse gender using Sly/XIr and Zfy PCR.

Notes: Genomic DNA from YY male and XY female mice were amplified with primers for (A) Y chromosome Sly intron 8 and (B) X chromosome Xlr intron 6 and Y chromosome Zfy. 
Table 4-3. Serum 25-(OH) $\mathrm{VD}_{3}$ values across groups (Representative data).

\begin{tabular}{ccc}
\hline Group & Gender & $\begin{array}{c}\mathbf{2 5 - ( O H ) ~ V D} \\
(\mathbf{n g} / \mathbf{m L})\end{array}$ \\
\hline CON & $\mathrm{F}$ & 102.8 \\
CON & $\mathrm{F}$ & 50.4 \\
CON & $\mathrm{M}$ & 87.6 \\
CON & $\mathrm{M}$ & 36.4 \\
VDD & $\mathrm{F}$ & 2.4 \\
VDD & $\mathrm{F}$ & 1.6 \\
VDD & $\mathrm{M}$ & 3.2 \\
VDD & $\mathrm{M}$ & 2.4 \\
\hline
\end{tabular}

Notes: $\mathrm{CON}=\mathrm{VD}_{3}$ sufficient, $\mathrm{VDD}=\mathrm{VD}_{3}$ deficient, $\mathrm{M}=$ Male, $\mathrm{F}=$ Female. 
Table 4-4. $\quad V_{3}$-sufficient (control), -deficient (VDD) male (M) mice complete blood count (CBC).

\begin{tabular}{|c|c|c|c|c|c|c|c|c|}
\hline \multirow[b]{2}{*}{ Cell type } & \multicolumn{2}{|c|}{ Control M } & \multicolumn{2}{|c|}{ VDD M } & \multicolumn{2}{|c|}{ Control M ND } & \multicolumn{2}{|c|}{ VDD M ND } \\
\hline & Average & S.D. & Average & S.D. & Average & S.D. & Average & S.D. \\
\hline WBC (X 103/uL) & 45.94 & 30.1 & 38.78 & 15.48 & 2.55 & 2.19 & 5.57 & 0.72 \\
\hline Neutrophil (\#) & 18.46 & 9.19 & 17.22 & 7.55 & 0.39 & 0.32 & 1.11 & 0.11 \\
\hline Lymphocyte (\#) & 15.43 & 13.26 & 10.78 & 4.97 & 1.97 & 1.71 & 4 & 0.73 \\
\hline Monocyte (\#) & 10.14 & 8.16 & 7.07 & 2.93 & 0.19 & 0.17 & 0.47 & 0.11 \\
\hline Eosinophil (\#) & 1.73 & 1.43 & 2.84 & 2.37 & 0.01 & 0.01 & 0.01 & 0.01 \\
\hline Basophil (\#) & 0.19 & 0.21 & 0.87 & 0.78 & 0.01 & 0.01 & 0 & 0 \\
\hline Nucleated RBC (\#) & 0 & 0 & 0 & 0 & 0 & 0 & 0 & 0 \\
\hline Neutrophil (\%) & 41.99 & 8.07 & 44.76 & 7.82 & 15.22 & 0.51 & 19.89 & 0.62 \\
\hline Lymphocyte (\%) & 32.74 & 7.56 & 28.37 & 8.85 & 76.98 & 0.74 & 71.46 & 3.83 \\
\hline Monocyte (\%) & 21.21 & 5.42 & 18.31 & 2.47 & 7.1 & 0.62 & 8.54 & 3.09 \\
\hline Eosinophil (\%) & 3.67 & 2.78 & 6.56 & 3.12 & 0.45 & 0.46 & 0.13 & 0.11 \\
\hline Basophil (\%) & 0.38 & 0.4 & 2 & 1.05 & 0.26 & 0.37 & 0 & 0 \\
\hline Nucleated RBC (\%) & 0 & 0 & 0 & 0 & 0 & 0 & 0 & 0 \\
\hline Hematocrit (\%) & 33.5 & 2.94 & 28 & 4.31 & 31.6 & 1.41 & 31.05 & 2.19 \\
\hline RBC (X 106/uL) & 8.03 & 0.71 & 6.63 & 0.91 & 7.89 & 0.45 & 7.55 & 0.91 \\
\hline Hemoglobin (g/dL) & 12.01 & 1.18 & 10.02 & 1.39 & 12.05 & 0.49 & 11.6 & 0.71 \\
\hline $\begin{array}{l}\text { Mean Corpuscular } \\
\text { Volume (fL) }\end{array}$ & 41.72 & 1.38 & 42.12 & 1.02 & 40.05 & 0.49 & 41.25 & 2.05 \\
\hline $\begin{array}{l}\text { Mean Corpuscular } \\
\text { Hemoglobin (pg) }\end{array}$ & 14.95 & 0.58 & 15.1 & 0.47 & 15.3 & 0.28 & 15.45 & 0.92 \\
\hline Mean Corpuscular & & & & & & & & \\
\hline $\begin{array}{l}\text { Hemoglobin Conc } \\
(\mathrm{g} / \mathrm{dL})\end{array}$ & 35.83 & 0.76 & 35.89 & 1.32 & 38.1 & 0.14 & 37.35 & 0.35 \\
\hline $\begin{array}{l}\text { Red Cell Distribution } \\
\text { Width }(\%)\end{array}$ & 13.45 & 0.91 & 12.77 & 0.34 & 13.7 & 0.57 & 13.95 & 0.49 \\
\hline RSD & 5.61 & 0.56 & 5.38 & 0.19 & 5.5 & 0.14 & 5.75 & 0.49 \\
\hline Reticulocyte (\#) & 14.69 & 17.73 & 4.32 & 5.03 & 9.85 & 12.8 & 8.95 & 12.66 \\
\hline Reticulocyte (\%) & 0.2 & 0.25 & 0.07 & 0.09 & 0.13 & 0.17 & 0.13 & 0.18 \\
\hline Platelet (X 103/uL) & 412.8 & 114.23 & 257.33 & 43.81 & 505.5 & 27.58 & 469.5 & 154.86 \\
\hline $\begin{array}{l}\text { Mean Platelet } \\
\text { Volume (fL) }\end{array}$ & 5.68 & 0.39 & 5.54 & 0.35 & 5 & 0 & 4.85 & 0.07 \\
\hline $\begin{array}{l}\text { Platelet Distribution } \\
\text { Width }\end{array}$ & 65.15 & 9.56 & 66.86 & 9.29 & 48.9 & 0.42 & 49.8 & 0.57 \\
\hline Plateletcrit & 0.24 & 0.08 & 0.14 & 0.03 & 0.25 & 0.01 & 0.23 & 0.07 \\
\hline
\end{tabular}

Notes: Control $=\mathrm{VD}_{3}$ sufficient, $\mathrm{VDD}=\mathrm{VD}_{3}$ deficient, $\mathrm{M}=$ Male, $\mathrm{ND}=$ No disease, S.D. $=$ Standard deviation.

Red font indicates higher levels compared to control group; Green font indicates lower levels compared to control group 
Table 4-5. $\quad V_{3} D_{3}$-sufficient and -deficient male mice serum chemistry.

\begin{tabular}{|c|c|c|c|c|c|c|c|c|}
\hline \multirow[b]{2}{*}{ Parameter } & \multicolumn{2}{|c|}{ Control M } & \multicolumn{2}{|c|}{ VDD M } & \multicolumn{2}{|c|}{ Control M ND } & \multicolumn{2}{|c|}{ VDD M ND } \\
\hline & Average & S.D. & Average & S.D. & Average & S.D. & Average & S.D. \\
\hline $\begin{array}{l}\text { Triglyceride } \\
(\mathrm{mg} / \mathrm{dl})\end{array}$ & 52.99 & 15.89 & 44.63 & 13.17 & 66.15 & 3.04 & 39.15 & 17.61 \\
\hline $\mathrm{HDL}(\mathrm{mg} / \mathrm{dl})$ & 47.24 & 12.71 & 58.46 & 10.49 & 87.15 & 6.58 & 45.25 & 42.78 \\
\hline LDL (mg/dl) & 9.15 & 1.99 & 18.31 & 16.24 & 8.45 & 0.49 & 4.10 & 3.25 \\
\hline $\begin{array}{l}\text { Cholesterol } \\
(\mathrm{mg} / \mathrm{dl})\end{array}$ & 102.82 & 27.20 & 116.00 & 16.24 & 139.50 & 3.54 & 73.00 & 70.71 \\
\hline
\end{tabular}

Notes: Control $=\mathrm{VD}_{3}$ sufficient, $\mathrm{VDD}=\mathrm{VD}_{3}$ deficient, $\mathrm{M}=$ male, $\mathrm{ND}=$ no disease, $\mathrm{S} . \mathrm{D}$. $=$ standard deviation, $\mathrm{HDL}=$ High-density lipoprotein, $\mathrm{LDL}=$ Low-density lipoprotein . Red font indicates higher levels compared to control group. 
Body weights of animals on day 1 of experiments 1and 2 were compared. The mean body weights $(\mathrm{g})$ for male vitamin sufficient $(21.58+1.95)$ versus. VDD $(21.5+1.99)$ mice, and female vitamin sufficient $(17.83+0.99)$, VDD $(17.09+1.35)$ mice were not different from control. In addition, bone vascularity, trabecular volume, and osteoblast and osteoclast numbers were evaluated on the same HE-stained longitudinal sections of tibia and no significant differences between the control and VDD group were observed.

\section{Bone Marrow and Spleen BCR-ABL Leukemia Burden is Lower in VDD Mice Compared to Vitamin D Sufficient Mice}

To further assess the relative leukemia burden at the primary sites of leukemia replication such as bone marrow and spleen. We quantified the number of luciferasetagged BCR-ABL cells in the bone marrow (Figure 4-9A). In addition, a blind scoring of the disease burden in H\&E stained histological sections of the hind limb and spleen was performed by the pathologist (Dr. Laura Janke, St. Jude Children's Research Hospital) (Figure 4-9B, 4-9C). Consistent with lower total leukemia body burden (Figure 4-6), VDD male mice had lower levels of bone marrow, hind limb and spleen leukemia.

\section{Mice Vitamin D Deficient for a Shorter Time Interval Still Show Increased Survival from BCR-ABL ALL Compared to Vitamin D-Sufficient Mice}

All of the mouse survival studies were performed on mice rendered vitamin deficient by placing pregnant females on vitamin $\mathrm{D}$ deficient diets during pregnancy and parturition and maintaining weaned pups on these deficient diets. We also used an alternate strategy to generate vitamin D deficiency in a shorter time frame and compared BCR-ABL ALL survival between vitamin D sufficient mice and mice rendered VDD by placing them on a VDD diet at 4 weeks (weaning), using them after 10 weeks of age. Despite the shorter duration of vitamin D deficiency, VDD mice still had prolonged survival from BCR-ABL Arf-/- ALL (Figure 4-10).

\section{$1,25(\mathrm{OH})_{2} \mathrm{VD}_{3}$ Increases the Number of BCR-ABL Arf-/- ALL Cells Only When Co- cultured with Bone Marrow Stromal Cells}

To begin exploring the mechanism of how BCR-ABL+ ALL was proliferating more rapidly in vitamin $D$ sufficient versus deficient mice (Figures 4-4, 4-5, 4-6), we examined the effect of $1,25-(\mathrm{OH})_{2} \mathrm{VD}_{3}$ treatment on BCR-ABL Arf-/- leukemia cells. Because mouse BCR-ABL+ Arf-/- leukemia cells, like human primary B-ALL cells (Consolini et al., 2001), lack VDR (Figure 4-11) required for mediating the $\mathrm{VD}_{3}$ signal, and because leukemic cells proliferate in the bone marrow, BCR-ABL+ B-ALL cells were co-cultured with immortalized human mesenchymal stromal cells (hMSCs) expressing VDR (Figure 4-11) that mimic a bone marrow micro-environment. There was no effect of $1,25(\mathrm{OH})_{2} \mathrm{VD}_{3}$ on number of viable BCR-ABL+ B-ALL cells grown without 


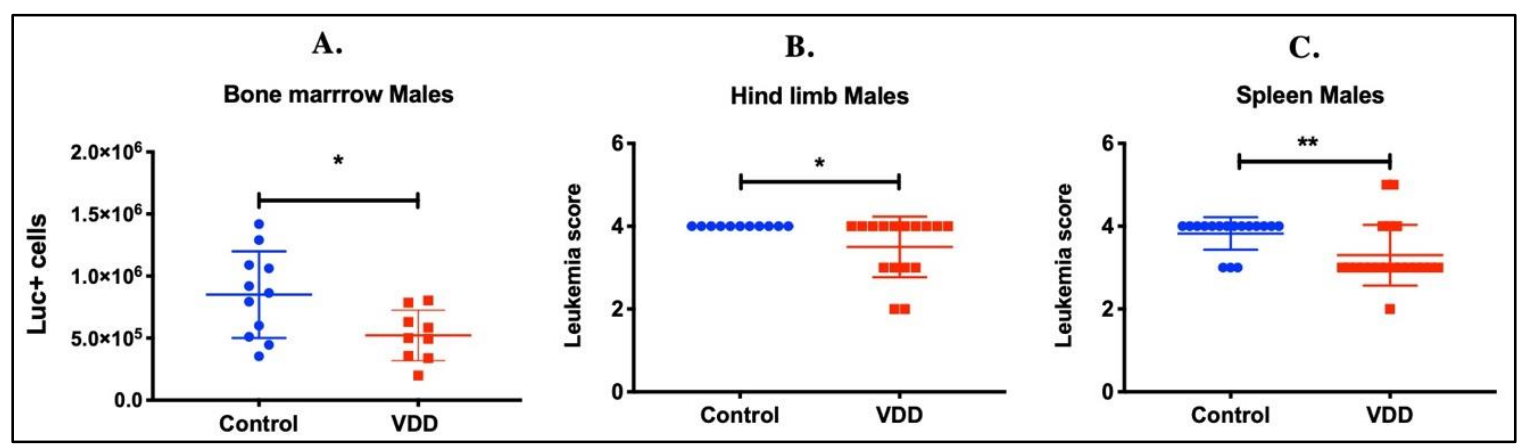

Figure 4-9. Luminescence measure and leukemia score in control and VDD male mice.

Notes: (A) Luminescence measure of BCR-ABL Luc ${ }^{+}$Bone marrow disease burden in male control and VDD mice, (B) Hind limb leukemia score in male control and VDD mice, (C) Spleen leukemia score in male control and VDD mice.

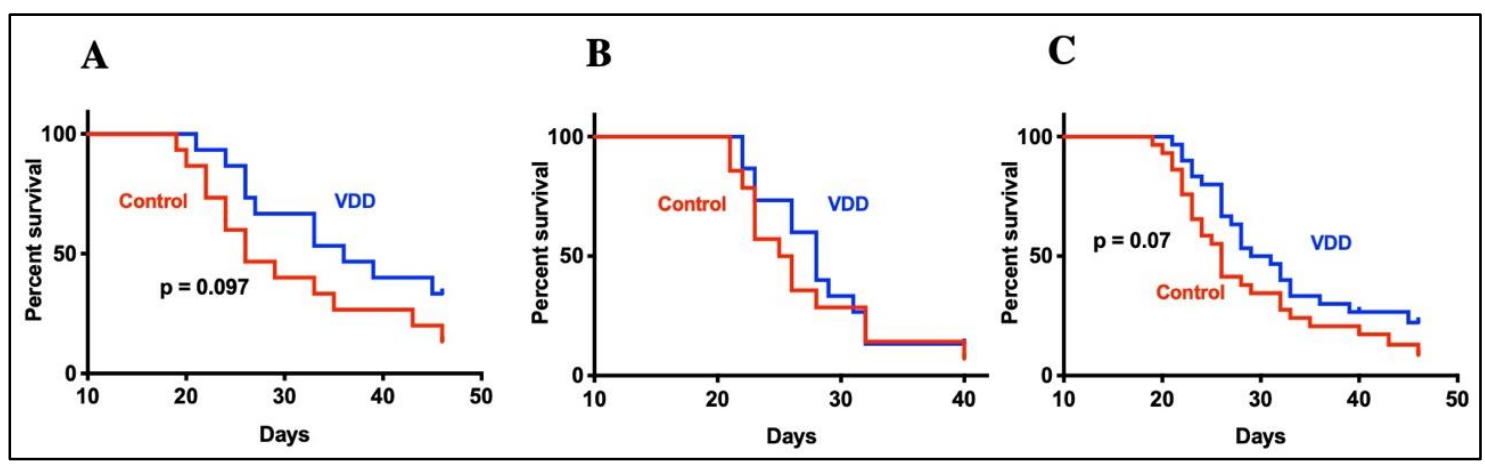

Figure 4-10. Kaplan meier survival curves using male mice started on control (vitamin D sufficient) and VDD diet beginning at weaning.

Notes: (A) Study 3 - Control ( $\mathrm{n}=15)$ versus VDD ( $\mathrm{n}=15)$, (B) Study 4- Control ( $\mathrm{n}=15)$ versus VDD ( $\mathrm{n}=15)$, (C) Study 3 and 4 combined curves. Differences between survival curves was determined using Gehan-Breslow-Wilcoxin test. The median survival for the control mice vs. VDD mice was 26 versus 36 days (A) and 26 versus 30 days (B). 


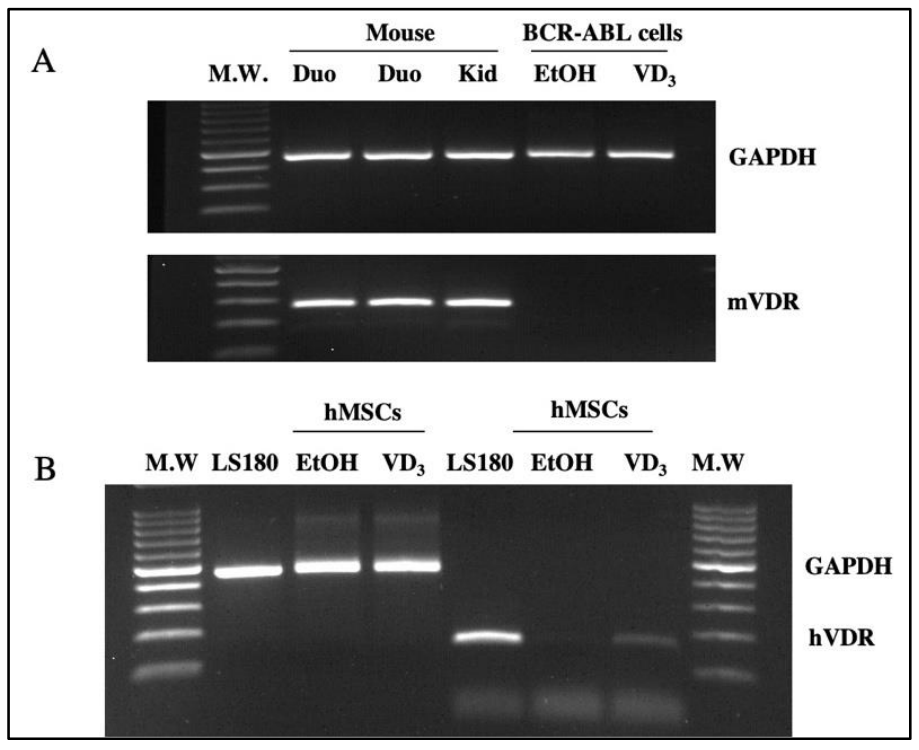

Figure 4-11. Determination of VDR expression using PCR.

Notes: (A) Lane 1: M.W, Lane 2-6 showing GAPDH band at 500bp and lane 2-4 (positive control: mouse duodenum, kidney) mVDR band at 300bp, no band in lane 5-6 for mVDR in BCR-ABL ALL cells, (B) Lane 2-4 showing GAPDH band at 500bp and lane 5 (positive control, LS180 cells) hVDR band at 190bp, lane 6: hMSCs treated with ethanol no band of hVDR, lane 7: hMSCs treated with $1,25(\mathrm{OH})_{2} \mathrm{VD}_{3}$ a band of $\mathrm{hVDR}$. 
hMSCs ( $\mathrm{p}=0.5972)$; however, $1,25(\mathrm{OH})_{2} \mathrm{VD}_{3}$ increased the number of BCR-ABL cells $(2.102 \pm 0.415$ - fold higher, $\mathrm{p}=0.012)$ ) (Figure 4-12) when co-cultured with hMSCs. This result suggests that $1,25(\mathrm{OH})_{2} \mathrm{VD}_{3}$ is acting through the stroma to enhance BCR-ABL ALL cell growth. In total, this result showed that $1,25(\mathrm{OH})_{2} \mathrm{VD}_{3}$ enhances BCR-ABL ALL cell number in vitro and this requires co-culture with bone marrow stroma.

\section{1, 25(OH $)_{2} \mathrm{VD}_{3}$ Increases BCR-ABL Cell Migration to hMSCs in Transwell Culture}

Since the in vivo vitamin D sufficient (control) mice showed higher ALL disease burden in the bone marrow, compared with the VDD mice (Figure 4-9) we next tested whether this also reflected vitamin D increasing migration of the leukemic blasts to the bone marrow stroma. An in vitro cell homing assay was used in which we measured the percentage of BCR-ABL leukemia cells migrating from the donor chamber of a transwell culture through a semi-permeable membrane to the bottom chamber containing media or containing hMSC conditioned with or without 1,25(OH) $2 \mathrm{VD}_{3}$ (Figure 4-13).

There was no effect of $1,25(\mathrm{OH})_{2} \mathrm{VD}_{3}$ on migration of ALL cells in the absence of hMSCs. However, BCR-ABL ALL cells were stimulated to migrate to hMSCs and a significantly greater percentage of BCR-ABL cells migrated to hMSCs conditioned for 48 hours with $100 \mathrm{nM} 1,25-(\mathrm{OH})_{2} \mathrm{VD}_{3}$ (Figure 4-13, ** $\mathrm{p}=0.007$ ). Fetal bovine serum (FBS), which alone can also provide the media with factors required for BCR-ABL cell migration, further increased the effect of $1,25-(\mathrm{OH})_{2} \mathrm{VD}_{3}(\mathrm{p}=0.064)$. This result demonstrates the ability of $1,25-(\mathrm{OH})_{2} \mathrm{VD}_{3}$ to enhance BCR-ABL cell migration is not intrinsic to the $\mathrm{BCR}-\mathrm{ABL}$ blast, but rather, is due to $\mathrm{VD}_{3}$-initiated reprogramming of the hMSCs to attract more BCR-ABL cells.

\section{$1,25(\mathrm{OH})_{2} \mathrm{VD}_{3}$ Increased CXCL12 mRNA and Protein Expression in hMSCs in vitro and in Mouse Serum in vivo}

CXCL12 (stromal cell-derived factor-1, Sdf-1) is a chemokine produced by stromal cells, endothelial cells and osteoblasts, that plays an important role in normal Bcell lymphopoesis, cell trafficking and homing to the bone marrow (L. Ding \& Morrison, 2013), and it has been shown to increase proliferation of primary pre-B-ALL (Mowafi et al., 2008). Induction of CXCL12 mRNA (Figure 4-14) expression was higher in hMSCs treated with $\mathrm{VD}_{3}$ for either 48 or 72 hours compared to control vehicle treated cells $(9.179 \pm 2.415$-fold $(* * * * p<0.0001)$ and $10.320 \pm 2.060$-fold $(* * * p=0.0002)$ increases, respectively.

Likewise, cell culture supernatants from hMSCs conditioned for 72 hours with $1,25-(\mathrm{OH})_{2} \mathrm{VD}_{3}$, had a 2-fold increase in secreted CXCL12 protein compared with vehicle treated controls (Figure 4-15A, ${ }^{*} p=0.029$ ). There was a trend toward a higher serum CXCL12 protein level in $\mathrm{VD}_{3}$ sufficient versus VD3 deficient mice (Figure 4-15B, $\mathrm{p}=0.095$ ). However, we did not find a significant difference in the CXCR4 (the 


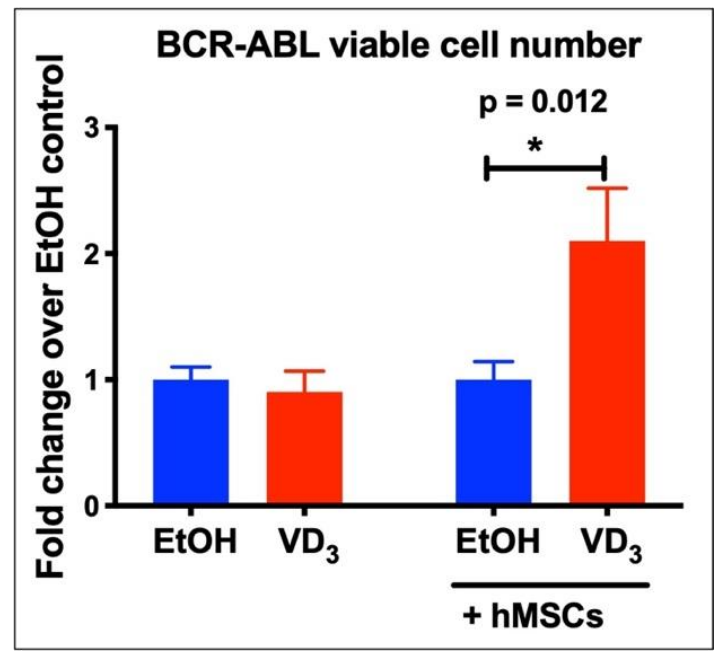

Figure 4-12. $1,25(\mathrm{OH})_{2} \mathrm{VD}_{3}$ increases $\mathrm{BCR}-\mathrm{ABL}$ proliferation in vitro.

Notes: The effect of $1,25(\mathrm{OH})_{2} \mathrm{VD}_{3}$ vs. vehicle treatment on growth of BCR-ABL ALL cell number when cultured in the absence or presence of hMSC and plotted as fold change relative to group control, $n=3$ per group. The unpaired t-test was used to compare differences in BCR-ABL cell number.

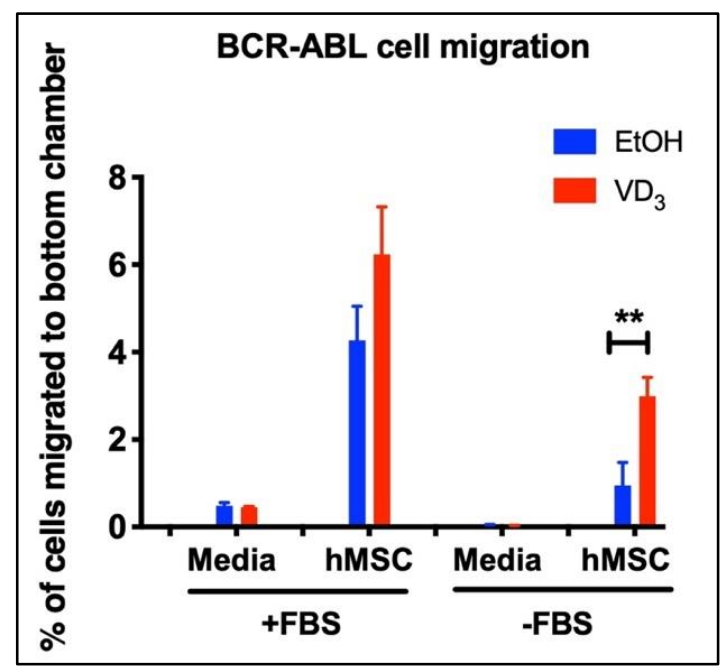

Figure 4-13. Increased migration of BCR-ABL leukemia cells to VD3 treated bone marrow stroma.

Notes: The percentage of the total number of BCR-ABL ALL Luc+ cells that migrated to the bottom chamber was determined. The unpaired t-test was used to compare increase in cell number $(* * \mathrm{p}<0.01)$. 


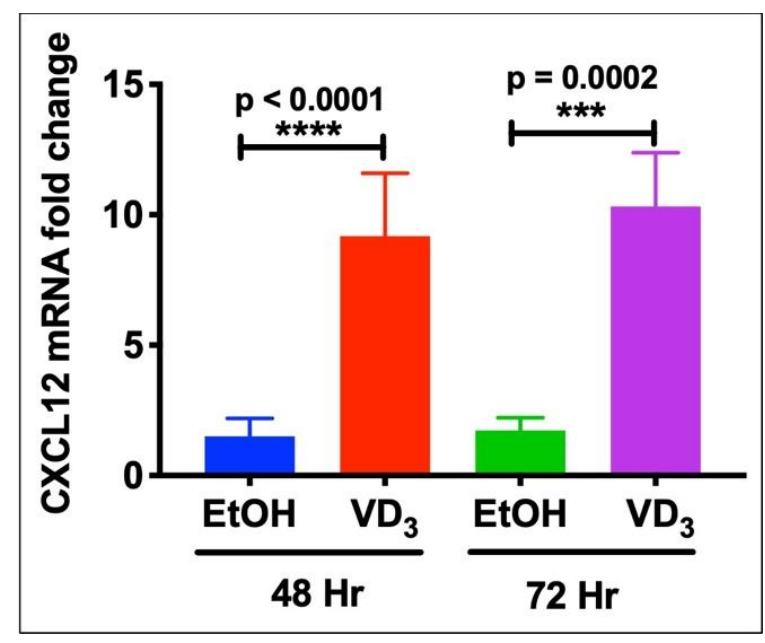

Figure 4-14. Vitamin D induces CXCL12 mRNA in bone marrow stroma in vitro.

Notes: CXCL12 mRNA transcripts in hMSC cells treated with ethanol (EtOH) or $100 \mathrm{nM}$ $1,25(\mathrm{OH})_{2} \mathrm{VD}_{3}, \mathrm{n}=8$ or 10 per group. Unpaired t-test was used to determine significance between the groups $(* * * \mathrm{p}<0.001, * * * * \mathrm{p}<0.0001)$.

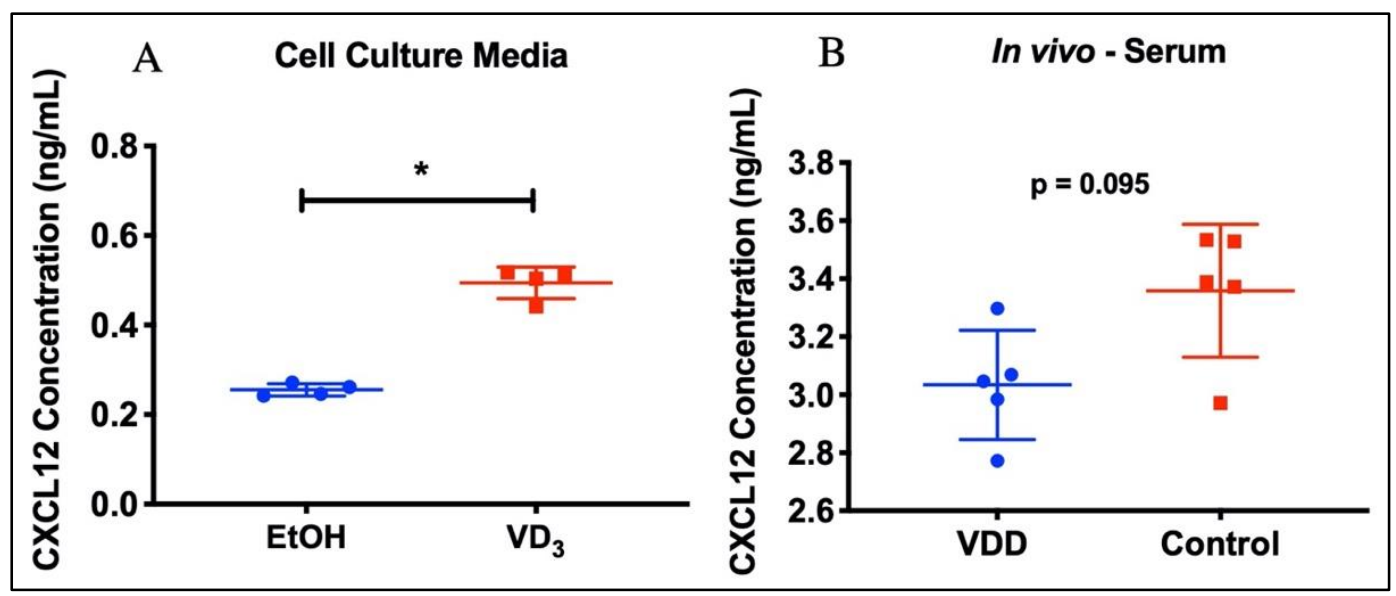

Figure 4-15. Vitamin D induces CXCL12 protein expression in bone marrow stroma in vitro and in serum of mice in vivo.

Notes: CXCL12 protein expression in the media of hMSC cells treated with ethanol $(\mathrm{EtOH})$ or $100 \mathrm{nM} 1,25(\mathrm{OH})_{2} \mathrm{VD}_{3}, \mathrm{n}=4$ per group. CXCL12 protein expression in the serum from $\mathrm{VD}_{3}$ sufficient (control) or VDD mice, $\mathrm{n}=5$ per group. Mann-Whitney nonparametric test on GraphPad was used to determine significance between the groups $(* p<0.05)$ 
CXCL12 receptor) expression in 1, 25- $(\mathrm{OH})_{2} \mathrm{VD}_{3}$ treated versus ethanol treated BCRABL ALL cells (not shown). Hence, $1,25-(\mathrm{OH})_{2} \mathrm{VD}_{3}$ reprograms the bone marrow stroma to produce and secrete CXCL12 to attract leukemia cells.

\section{CXCL12 Increases the Number of BCR-ABL Cells in Suspension Culture}

To determine whether CXCL12 could directly affect BCR-ABL cells, we treated cells in culture with CXCL12. Since VD 3 stimulated hMSC cells produced $0.5 \mathrm{ng} / \mathrm{ml}$ of CXCL12 (Figure 4-16), BCR-ABL cells in suspension culture, or co-cultured with hMSCs, were treated with $0.5 \mathrm{ng} / \mathrm{ml}$ CXCL12 for 48 hours. CXCL12 produced a significant increase $\left({ }^{*} p=0.04\right)$ in BCR-ABL cell number in cells in suspension culture, but no further increase in the number of viable BCR-ABL cells co-cultured with hMSC. This demonstrates that exogenous CXCL12 can directly stimulate growth of BCR-ABL leukemia cells.

\section{Vitamin D Plus ALL Reprograms the Bone Marrow Stroma}

A growing body of literature demonstrates a role for $\mathrm{VD}_{3}$ in remodeling stroma that can then affect cancer growth and chemotherapeutic response (Mowafi et al., 2008). Similarly, the presence of leukemia in the bone marrow has been shown to transform the bone marrow niche into a leukemia-permissive microenvironment causes reprogramming (B. Kumar et al., 2018).

To test whether vitamin $\mathrm{D}$ remodels the extracellular matrix production by bone marrow stroma, immunohistochemistry for type I and V collagen was performed on the bone marrow of healthy vitamin D sufficient and deficient mice, as well as on those with BCR-ABL ALL obtained at the time of sacrifice. Both type I and V collagen were minimally detectable in the bone marrow of healthy mice, regardless of vitamin D levels, but increased in the marrow of mice with ALL, but more frequently in the VD3 sufficient (control) group (Figure 4-17). Hence, the presence of BCR-ABL together with vitamin D is reprogramming the bone marrow stroma to increase production of type I and $\mathrm{V}$ collagens.

\section{Vitamin D Deficiency Alters the Number of CXCL12 Expressing Cell Types in Bone Marrow and Bone}

To evaluate if vitamin D deficiency had any effect on the bone marrow microenvironment, we evaluated the abundance of different cells that express CXCL12 in bone tissue and bone marrow. In vitro vitamin D induced CXCL12 expression in hMSCs, but in vivo many other cells types such as endothelial cells, osteoblasts, perivascular stromal cells and CXCL12 abundant reticular cells (CAR) also significantly express CXCL12. We analyzed the percentage of these cell types by flow cytometry in vitamin D deficient versus sufficient mouse bone and bone marrow tissues. We observed that, $\mathrm{VD}_{3}$ 


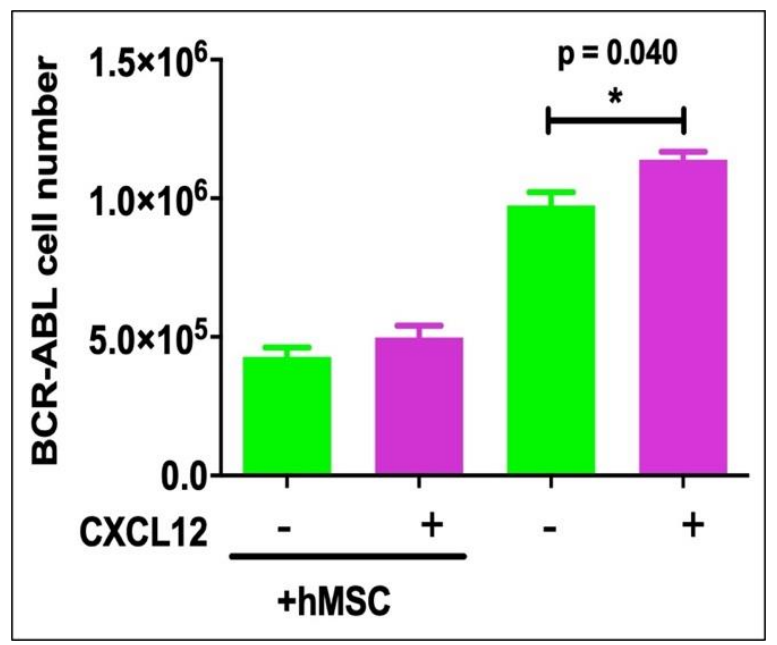

Figure 4-16. CXCL12 increases proliferation of BCR-ABL ALL cells in suspension culture.

Notes: Effect of CXCL12 treatment (48 hr) on BCR-ABL cell number in cells cultured alone or with hMSCs, $\mathrm{n}=3$ per group. The unpaired t-test was used to compare increase in cell number $(* \mathrm{p}<0.05)$

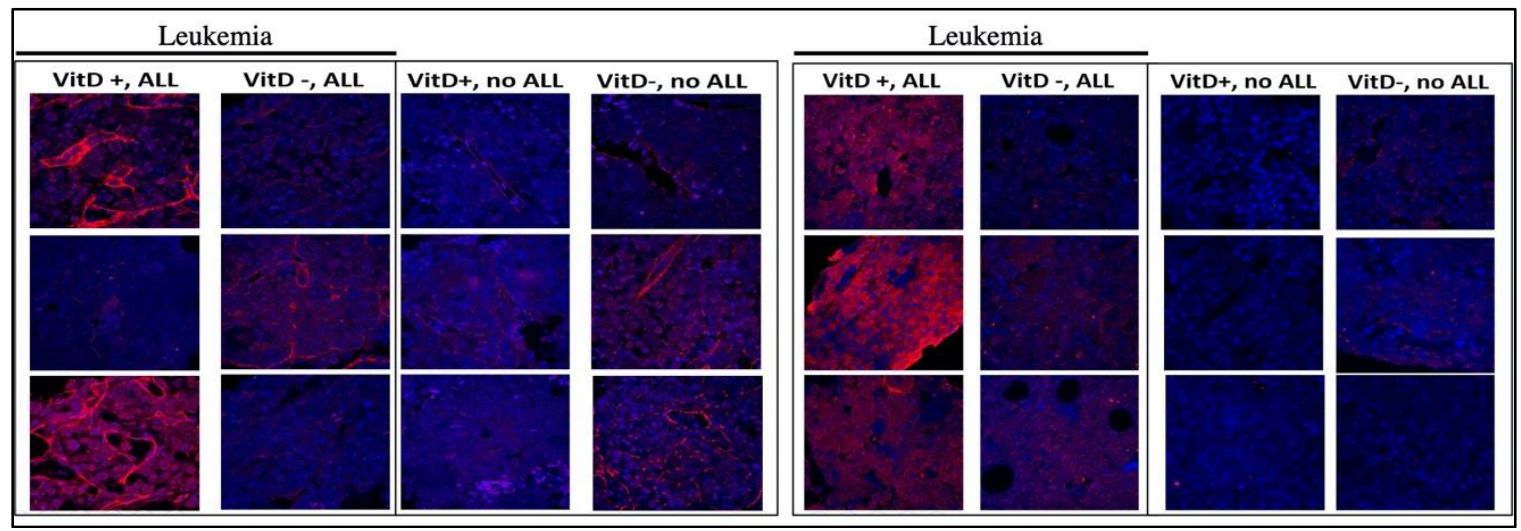

Figure 4-17. Effect of vitamin $D$ and BCR-ABL leukemia on expression of type 1 and type $\mathrm{V}$ collagens in mouse bone marrow.

Notes: Fluorescent immunohistochemistry was performed on paraffin embedded mouse tibias to examine the expression of collagens 1 and $\mathrm{V}$ in vivo. Collagens 1 and $\mathrm{V}$ both show the highest expression in $\mathrm{VD}_{3}$ sufficient mice with ALL. 
deficient mice had lower percentage of CD31 negative cells (Figure 4-18) in the bone marrow which consists of all the CXCL12 expressing cells except endothelial cells. The percentage of CXCL12 expressing cell types $\mathrm{P} \alpha \mathrm{S}$ stromal cells $(\mathrm{P} \alpha \mathrm{S})$, mesenchymal stem cells (MSCs), osteoblasts and CAR cells in bone marrow was low in the bone marrow of vitamin D deficient mice compared to the sufficient mice. We also observed a significant effect of vitamin D deficiency in the bone tissue where CD31 negative cell percentage was significantly low compared to sufficient mice (Figure 4-19). However, $\mathrm{P} \alpha \mathrm{S}, \mathrm{MSCs}$ and osteoblasts, although they make up a very small percentage of CD31 negative cells, were significantly higher in $\mathrm{VD}_{3}$ deficient mice compared to sufficient mice. But CARs are the most abundant CD31 negative cells making up to 25-30\% of CD31 negative cells and are significantly lower in $\mathrm{VD}_{3}$ deficient bones (Figure 4-19).

\section{Discussion}

Despite the high frequency of vitamin D deficiency in humans worldwide, this is the first study to test and demonstrate that host deficiencies in vitamin $\mathrm{D}$ can affect growth of BCR-ABL ALL Arf-/- leukemia and survival of mice from this disease. Our study found that vitamin D sufficient mice had markedly accelerated disease progression and decreased survival from BCR-ABL ALL compared to mice that were vitamin D deficient. A similar effect of vitamin D deficiency on survival from BCR-ABL ALL was seen regardless of whether mice were exposed to a VDD diet during gestation (Figure 4 -7) or started the diet at weaning (Figure 4-10), demonstrating the effect vitamin D deficiency on BCR-ABL leukemogenesis in this mouse model does not require deficiency during gestation.

Surprisingly, host $\mathrm{VD}_{3}$ sufficiency versus deficiency was detrimental to host survival from BCR-ABL Arf-/- ALL. Importantly, 1,25 $(\mathrm{OH})_{2} \mathrm{VD}_{3}$ had no effect on growth of the leukemia cells in suspension culture but required co-culture with the bone marrow stroma. This result is consistent with the apparent absence of the VDR in mouse BCR-ABL+ ALL Arf-/- leukemia cells, and similarly its absence in human primary BALL cells (Consolini et al., 2001).

Our results also offer a cautionary reminder for the many studies that test the effect of chemicals on leukemia cells grown in suspension culture, without co-culture of bone marrow stromal cells, as these studies may miss important leukemia cell responses that are only seen in a co-culture setting. Bone marrow stroma cells are known to form a microenvironment not only conducive for leukemic cell growth, but that can also regulate responsiveness to anti-leukemic therapies such as asparaginase and tyrosine kinase inhibitors (Iwamoto et al., 2007; B. Zhang et al., 2013).

Our results extend this paradigm to the effect of $1,25-(\mathrm{OH})_{2} \mathrm{VD}_{3}$ on growth and migration of BCR-ABL+ ALL Arf-/- leukemia cells. 1, 25- $(\mathrm{OH})_{2} \mathrm{VD}_{3}$ actively induced bone marrow stroma to release CXCL12 in vitro, and increased CXCL12 serum levels in vivo, resulting in an increased ability to attract the BCR-ABL+ Arf-/- ALL to the marrow and support its growth. Different cell types in the bone marrow, other than hMSCs that 


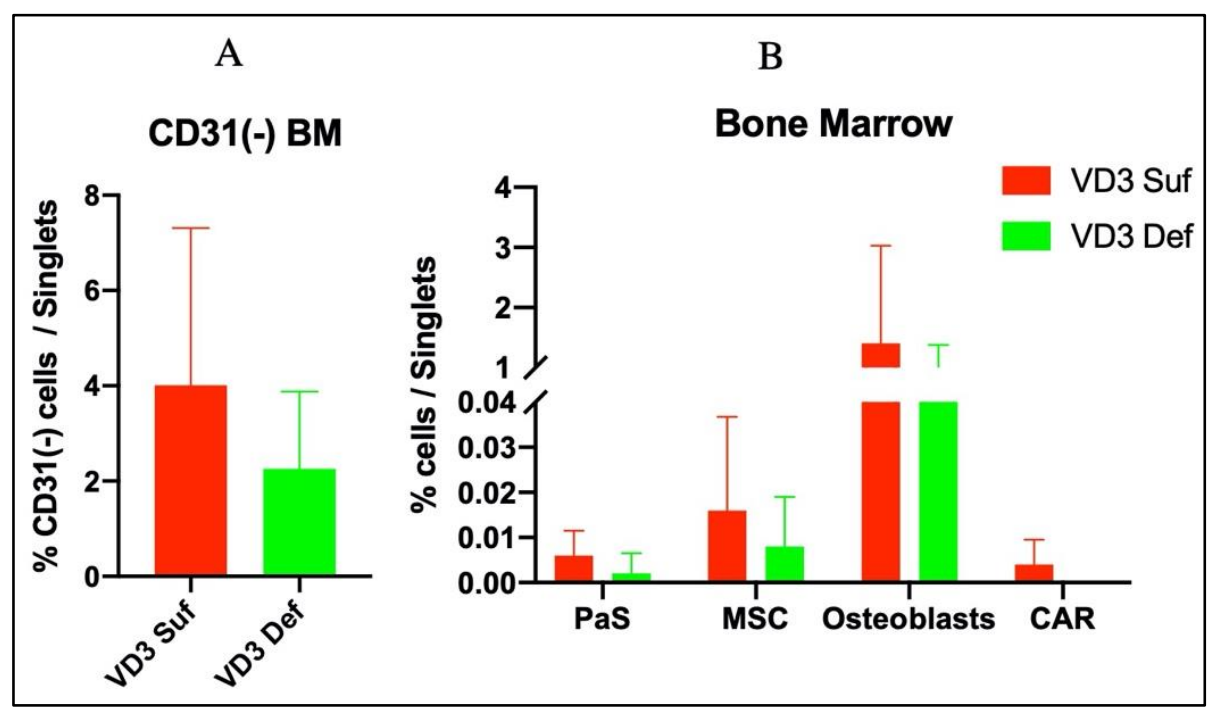

Figure 4-18. Percentage of different CXCL12 expressing cells in bone marrow of VD 3 -sufficient mice versus $V_{3}$-deficient mice.

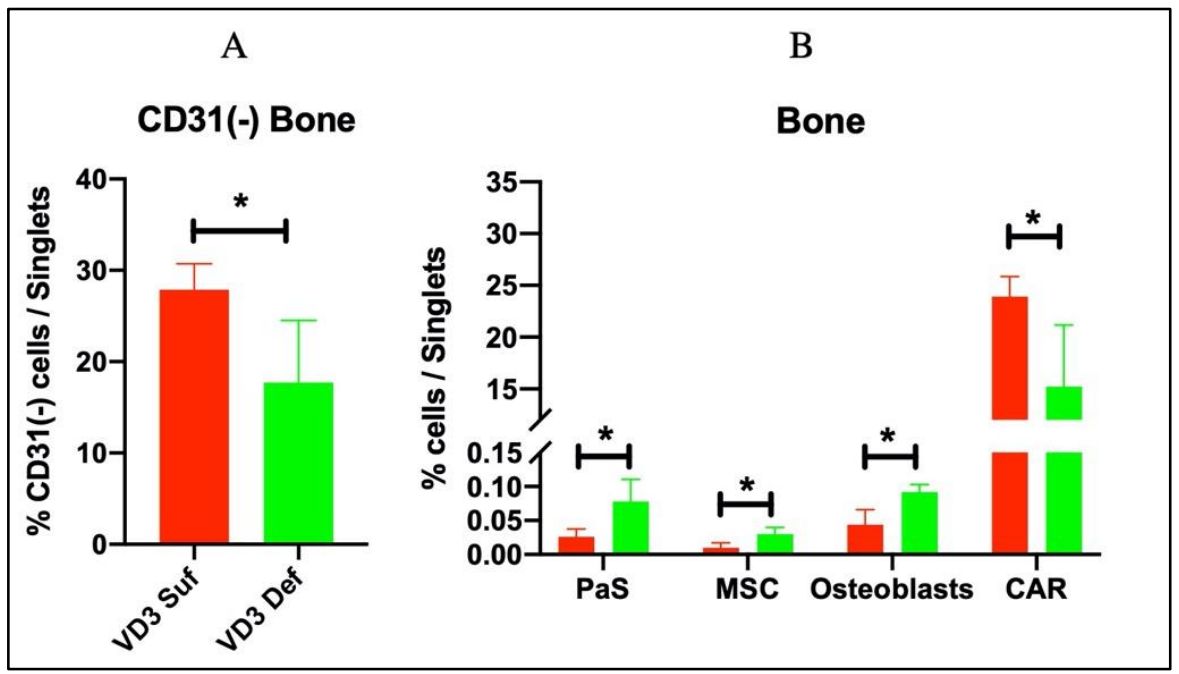

Figure 4-19. Percentage of different CXCL12 expressing cells in bone tissue of $V_{3}$-sufficient mice versus $V_{3}$-deficient mice. 
produce CXCL12 may also be responsive to $1,25-(\mathrm{OH})_{2} \mathrm{VD}_{3}$ and increase the production of chemokine CXCL12. Apart from hMSCs, in vivo multiple other cell types such as PaS mesenchymal stem cells, osteoblasts, endothelial cells and CARs express CXCL12.

Above all, CARs express significant amount of CXCL12 in the bone marrow microenvironment. In bone and bone marrow of vitamin D sufficient mice CAR cell percentage is significantly higher compared to $\mathrm{VD}_{3}$ deficient mice. Our results suggest that vitamin D not only can induce CXCL12 expression in vitro, but in vivo vitamin D can significantly affect the number of CXCL12 expressing cells.

In vivo, vitamin $\mathrm{D}$, in the presence of $\mathrm{BCR}-\mathrm{ABL}+$ Arf-/- leukemia, also remodeled the bone marrow stroma, inducing expression of Type 1 and $\mathrm{V}$ collagens. Others have reported that increased bone marrow reticulin and collagen fibers are associated with malignant diseases and may anchor and trap the leukemic cells in the marrow providing an environment where they become more drug resistant (Nath et al., 2011). While this study probed the interaction of $1,25-(\mathrm{OH})_{2} \mathrm{VD}_{3}$ signaling through the co-cultured bone marrow stromal cells, in vivo multiple other cell types in the bone marrow express VDR, including osteoblasts. Hence, in vivo, Vitamin D may signal through VDR in additional cells in the bone and bone marrow influencing the microenvironment and differentially altering the growth and viability of the leukemia cells.

It will be important to determine if vitamin $\mathrm{D}$ levels significantly affect the growth of BCR-ABL+ Arf-/- ALL in mice in vivo can be extended to other subtypes of pediatric leukemia. Moreover, do these results in mice translate to humans? Interestingly, a study in children that measured dietary vitamin $\mathrm{D}$ intake in newly diagnosed children with ALL found that vitamin D intake was significantly different between patients who presented with high-risk ALL versus standard risk ALL. Failure to meet daily dietary amounts of vitamin D was higher in the standard risk versus high-risk patients, while the proportion of patients who met the vitamin D dietary requirements was higher in the high risk versus standard risk patients (Ladas et al., 2016). Clearly our findings suggest that the clinical levels of vitamin D should be measured in leukemic patients over the course of therapy to determine if there is an association in humans between vitamin sufficiency and survival from different subtypes of leukemia, and if vitamin supplementation to vitamin deficient patients affects survival from acute lymphoblastic leukemias. 


\section{CHAPTER 5. SUMMARY}

The overall goal of this research work was to evaluate if vitamin D deficiency could cause any vitamin - drug interaction with CYP3A4 substrates and if it has an effect on survival outcome from B-ALL. We developed diet-based age-controlled vitamin D sufficient and deficient mice and evaluated changes associated with vitamin D status on intestinal, hepatic and renal drug metabolizing enzymes (CYP3A) and drug transporter (PGP, BCRP, MRP4). We found that dietary vitamin D can alter mouse intestinal Cyp3a11 and hCYP3A4 expression in female mice. Paradoxically, we also observed a significant increase in intestinal transporter expression in both male and female vitamin D deficient mice. However, these changes did not cause a significant vitamin drug interaction with dexamethasone and dasatinib which are both CYP3A4 and PGP substrates and given orally to ALL patients. However, we found that vitamin D sufficient mice had markedly accelerated disease progression and decreased survival from BCRABL ALL compared to mice that were vitamin D deficient.

DEX is a potential inducer of CYP3A depending on the concentrations achieved in the tissue. We calculated gut concentrations of DEX to be in sub-micromolar range and can activate PXR to induce CYP3A genes. Utilizing intestinal and liver tissues from DEX treated mice for 3.5 days, we analyzed the expression of CYP genes. Our results suggest that, 3.5 days treatment of DEX has significantly induced multiple CYP3A genes in the intestine of vitamin D deficient group but not the sufficient group. Moreover, we analyzed the expression of CYP3A and CYP2B genes in the livers of DEX treated mice. The plasma concentrations of DEX in this study was in nanomolar range, which can activate GR but not PXR. We observed significant induction of CYP2B genes in all the groups indicating GR activation in the livers.

When we placed vitamin D sufficient and deficient mice injected with BCR-ABL ALL cells on discontinuous DEX regimen, despite the treatment, vitamin D sufficient mice fared worse than the vitamin D deficient mice. This indicated that vitamin D itself has an effect on BCR-ABL leukemia progression. Therefore, we next evaluated the mechanisms by which vitamin D can affect leukemia cells.

Our studies show that BCR-ABL ALL cells do not express VDR, a receptor through which vitamin D binds and regulates gene transcription. However, many cells in the bone marrow microenvironment in which the lymphoblasts proliferate in vivo, such as osteoblasts, endothelial cells and CARs express VDR. In vitro the BCR-ABL ALL cells are co-cultured in with hMSCs, which act as a bone marrow micro-environment, and these cells do express VDR. Co-culturing BCR-ABL cells with hMSCs and treating with vitamin D directly increased the proliferation of BCR-ABL ALL cells and increased their migration to co-cultured hMSCs. The increased migration of BCR-ABL ALL cells to hMSCs was mediated by Vitamin D induction of CXCL12, an important chemokine for leukemia cell proliferation and migration to hMSCs. In vivo, vitamin $\mathrm{D}$, in the presence of BCR-ABL+ Arf-/- leukemia, also remodeled the bone marrow stroma, inducing expression of Type 1 and $\mathrm{V}$ collagens, and further increased the number of CARs 
(CXCL12 producing cells) in the bone and bone marrow. Consequently, Vitamin D may signal through VDR in neighboring supportive cells in the bone and bone marrow, remodeling the microenvironment and differentially altering the growth and viability of the leukemia cells.

Our studies have highlighted that vitamin D deficiency has a significant effect on survival outcome. Although many retrospective reports suggest the association of vitamin D levels and cancer risk, since their primary end point was not overall survival or mortality, it will be important to design individual clinical trials to ascertain the association of vitamin D levels and survival outcome. Since many pediatric leukemia patients are given vitamin D supplementation, it is important to understand the impact of VD 3 supplementation during chemotherapy on survival and drug efficacy in patients with pre-B cell ALL. Further efforts are needed to elucidate vitamin D's role and potential utility in the clinical setting for different leukemia subtypes.

Clearly our findings suggest that the clinical levels of vitamin D should be measured in leukemic patients over the course of therapy to determine if there is an association in humans between vitamin sufficiency and survival from different subtypes of acute lymphoblastic leukemia, and if vitamin supplementation to vitamin deficient patients affects survival from acute lymphoblastic leukemias.

With vitamin D having a significant effect on BCR-ABL ALL cell proliferation and migration, it will be important to determine if vitamin D levels also affect other subtypes of pediatric leukemia. Moreover, there are many studies suggesting different roles of vitamin $\mathrm{D}$ in mice versus humans. Thus, it will be important to study if these results in mice translate to humans.

Our data demonstrate that dietary vitamin D can influence intestinal mouse Cyp3a11 and human CYP3A4 expression. Given that the level of induction of CYP3A genes is different between vitamin D deficient and sufficient mice, further studies investigating possible drug-drug interactions that could arise when CYP3A4 drugs are co-administered with DEX in vitamin D deficient condition could be of significant importance. This data might help in better understanding of clinical situations where multiple drugs are co-administered during various stages of chemotherapy.

The broad expression pattern and widespread effects of vitamin D signaling can lead to unexpected outcomes and caution might be needed while supplementing patients with vitamin $\mathrm{D}$. Owing to the known pleiotropic roles of vitamin $\mathrm{D}$, and taking into consideration unexpected results from this study showing vitamin D deficient mice have a survival advantage from ALL, as mentioned before it is important to study further effects of vitamin D deficiency and supplementation in other B-cell leukemia subtypes and other diseases. Vitamin D was shown to cause stromal cell remodeling in pancreatic cancer (Sherman et al., 2014), fibrosis in hepatic stellate cancer (N. Ding et al., 2013) and in this study altering the bone marrow microenvironment suggesting an indirect role of vitamin D on disease outcome. Although it has been reported that high vitamin D levels or intake of vitamin D reduces the risk of colorectal, breast cancer as well as type I 
diabetes, multiple sclerosis and hypertension (Holick \& Chen, 2008), it is critical to reevaluate any effects of vitamin D status on the disease progression and outcome as well as to discern the mechanistic pathways altered by vitamin D status which directly or indirectly can affect the disease outcome. This would be best performed through clinical trials that would evaluate, first, whether vitamin D status (without supplementation) effects disease progression or survival across ALL subtypes before beginning any vitamin $\mathrm{D}$ therapeutic treatment in these patients. 


\section{LIST OF REFERENCES}

Abe, E., Miyaura, C., Sakagami, H., Takeda, M., Konno, K., Yamazaki, T., . . Suda, T. (1981). Differentiation of mouse myeloid leukemia cells induced by 1 alpha,25dihydroxyvitamin D3. Proc Natl Acad Sci U S A, 78(8), 4990-4994. doi:10.1073/pnas.78.8.4990

Achour, B., Barber, J., \& Rostami-Hodjegan, A. (2014). Expression of hepatic drugmetabolizing cytochrome p450 enzymes and their intercorrelations: a metaanalysis. Drug Metab Dispos, 42(8), 1349-1356. doi:10.1124/dmd.114.058834

Allen, J. D., Brinkhuis, R. F., Wijnholds, J., \& Schinkel, A. H. (1999). The mouse Bcrp1/Mxr/Abcp gene: amplification and overexpression in cell lines selected for resistance to topotecan, mitoxantrone, or doxorubicin. Cancer Res, 59(17), 42374241. Retrieved from https://www.ncbi.nlm.nih.gov/pubmed/10485464

Annu, K., Cline, C., Yasuda, K., Ganguly, S., Pesch, A., Cooper, B., . . Schuetz, E. G. (2020). Role of Vitamins A and D in BCR-ABL Arf(-/-) Acute Lymphoblastic Leukemia. Sci Rep, 10(1), 2359. doi:10.1038/s41598-020-59101-4

Antony, R., Sheng, X., Ehsanipour, E. A., Ng, E., Pramanik, R., Klemm, L., . . . Mittelman, S. D. (2012). Vitamin D protects acute lymphoblastic leukemia cells from dexamethasone. Leuk Res, 36(5), 591-593. doi:10.1016/j.leukres.2012.01.011

Bailey, D. G., Malcolm, J., Arnold, O., \& Spence, J. D. (1998). Grapefruit juice-drug interactions. Br J Clin Pharmacol, 46(2), 101-110. doi:10.1046/j.13652125.1998.00764.x

Berridge, M. J. (2015). Vitamin D cell signalling in health and disease. Biochem Biophys Res Commun, 460(1), 53-71. doi:10.1016/j.bbrc.2015.01.008

Berridge, M. J. (2017). Vitamin D deficiency accelerates ageing and age-related diseases: a novel hypothesis. J Physiol, 595(22), 6825-6836. doi:10.1113/JP274887

Bhardwaj, R. K., Glaeser, H., Becquemont, L., Klotz, U., Gupta, S. K., \& Fromm, M. F. (2002). Piperine, a major constituent of black pepper, inhibits human Pglycoprotein and CYP3A4. J Pharmacol Exp Ther, 302(2), 645-650. doi:10.1124/jpet.102.034728

Bikle, D. D. (2012). Vitamin D and the skin: Physiology and pathophysiology. Rev Endocr Metab Disord, 13(1), 3-19. doi:10.1007/s11154-011-9194-0

Bikle, D. D. (2016). The Endocrine Society Centennial: Extrarenal Production of 1,25 Dihyroxyvitamin D Is Now Proven. Endocrinology, 157(5), 1717-1718. doi:10.1210/en.2016-1186

Bischoff-Ferrari, H. A., Willett, W. C., Orav, E. J., Lips, P., Meunier, P. J., Lyons, R. A., ... Dawson-Hughes, B. (2012). A pooled analysis of vitamin D dose requirements for fracture prevention. $N$ Engl J Med, 367(1), 40-49. doi:10.1056/NEJMoa1109617

Bittenbring, J. T., Neumann, F., Altmann, B., Achenbach, M., Reichrath, J., Ziepert, M., . . . Pfreundschuh, M. (2014). Vitamin D deficiency impairs rituximab-mediated cellular cytotoxicity and outcome of patients with diffuse large B-cell lymphoma treated with but not without rituximab. J Clin Oncol, 32(29), 3242-3248. doi:10.1200/JCO.2013.53.4537 
Blazsek, I., Farabos, C., Quittet, P., Labat, M. L., Bringuier, A. F., Triana, B. K., . . Misset, J. L. (1996). Bone marrow stromal cell defects and 1 alpha,25dihydroxyvitamin D3 deficiency underlying human myeloid leukemias. Cancer Detect Prev, 20(1), 31-42. Retrieved from https://www.ncbi.nlm.nih.gov/pubmed/8907201

Borst, P., \& Schinkel, A. H. (2013). P-glycoprotein ABCB1: a major player in drug handling by mammals. J Clin Invest, 123(10), 4131-4133. doi:10.1172/JCI70430

Boscoe, F. P., \& Schymura, M. J. (2006). Solar ultraviolet-B exposure and cancer incidence and mortality in the United States, 1993-2002. BMC Cancer, 6, 264. doi:10.1186/1471-2407-6-264

Cao, H., Xu, Y., de Necochea-Campion, R., Baylink, D. J., Payne, K. J., Tang, X., . . . Chen, C. S. (2017). Application of vitamin D and vitamin D analogs in acute myelogenous leukemia. Experimental Hematology, 50, 1-12. doi:10.1016/j.exphem.2017.01.007

Carlberg, C., \& Campbell, M. J. (2013). Vitamin D receptor signaling mechanisms: integrated actions of a well-defined transcription factor. Steroids, 78(2), 127-136. doi:10.1016/j.steroids.2012.10.019

Cascorbi, I. (2011). P-glycoprotein: tissue distribution, substrates, and functional consequences of genetic variations. Handb Exp Pharmacol(201), 261-283. doi:10.1007/978-3-642-14541-4_6

Chai, S. C., Cherian, M. T., Wang, Y. M., \& Chen, T. (2016). Small-molecule modulators of PXR and CAR. Biochim Biophys Acta, 1859(9), 1141-1154. doi:10.1016/j.bbagrm.2016.02.013

Chen, Y., Agarwal, S., Shaik, N. M., Chen, C., Yang, Z., \& Elmquist, W. F. (2009). Pglycoprotein and breast cancer resistance protein influence brain distribution of dasatinib. J Pharmacol Exp Ther, 330(3), 956-963. doi:10.1124/jpet.109.154781

Cheung, C., Yu, A. M., Chen, C. S., Krausz, K. W., Byrd, L. G., Feigenbaum, L., . . . Gonzalez, F. J. (2006). Growth hormone determines sexual dimorphism of hepatic cytochrome P450 3A4 expression in transgenic mice. J Pharmacol Exp Ther, 316(3), 1328-1334. doi:10.1124/jpet.105.094367

Choudhary, A., Chou, J., Heller, G., \& Sklar, C. (2013). Prevalence of vitamin D insufficiency in survivors of childhood cancer. Pediatr Blood Cancer, 60(7), 1237-1239. doi:10.1002/pbc.24403

Christakos, S., Dhawan, P., Verstuyf, A., Verlinden, L., \& Carmeliet, G. (2016). Vitamin D: Metabolism, Molecular Mechanism of Action, and Pleiotropic Effects. Physiol Rev, 96(1), 365-408. doi:10.1152/physrev.00014.2015

Christopher, L. J., Cui, D., Wu, C., Luo, R., Manning, J. A., Bonacorsi, S. J., . . Iyer, R. A. (2008). Metabolism and disposition of dasatinib after oral administration to humans. Drug Metab Dispos, 36(7), 1357-1364. doi:10.1124/dmd.107.018267

Collier, S. D., Wu, W. J., \& Pruett, S. B. (1998). Endogenous glucocorticoids induced by a chemical stressor (ethanol) cause apoptosis in the spleen in B6C3F1 female mice. Toxicol Appl Pharmacol, 148(1), 176-182. doi:10.1006/taap.1997.8324

Colmone, A., Amorim, M., Pontier, A. L., Wang, S., Jablonski, E., \& Sipkins, D. A. (2008). Leukemic cells create bone marrow niches that disrupt the behavior of normal hematopoietic progenitor cells. Science, 322(5909), 1861-1865. doi:10.1126/science. 1164390 
Consolini, R., Pala, S., Legitimo, A., Crimaldi, G., Ferrari, S., \& Ferrari, S. (2001). Effects of vitamin D on the growth of normal and malignant B-cell progenitors. Clin Exp Immunol, 126(2), 214-219. Retrieved from https://www.ncbi.nlm.nih.gov/pubmed/11703363

Cortes, M., Chen, M. J., Stachura, D. L., Liu, S. Y., Kwan, W., Wright, F., . . North, T. E. (2016). Developmental Vitamin D Availability Impacts Hematopoietic Stem Cell Production. Cell Rep, 17(2), 458-468. doi:10.1016/j.celrep.2016.09.012

Dame, M. C., Pierce, E. A., \& DeLuca, H. F. (1985). Identification of the porcine intestinal 1,25-dihydroxyvitamin D3 receptor on sodium dodecyl sulfate/polyacrylamide gels by renaturation and immunoblotting. Proc Natl Acad Sci U S A, 82(23), 7825-7829. doi:10.1073/pnas.82.23.7825

DeLuca, H. F., \& Schnoes, H. K. (1976). Metabolism and mechanism of action of vitamin D. Annu Rev Biochem, 45, 631-666. doi:10.1146/annurev.bi.45.070176.003215

Demirsoy, U., Sarper, N., Aylan Gelen, S., Zengin, E., Kum, T., \& Demir, H. (2017). The Association of Oral Vitamin D and Calcium Supplementation With Bone Mineral Density in Pediatric Acute Lymphoblastic Leukemia Patients. J Pediatr Hematol Oncol, 39(4), 287-292. doi:10.1097/MPH.0000000000000797

Ding, L., \& Morrison, S. J. (2013). Haematopoietic stem cells and early lymphoid progenitors occupy distinct bone marrow niches. Nature, 495(7440), 231-235. doi:10.1038/nature11885

Ding, L., Saunders, T. L., Enikolopov, G., \& Morrison, S. J. (2012). Endothelial and perivascular cells maintain haematopoietic stem cells. Nature, 481(7382), 457462. doi:10.1038/nature10783

Ding, N., Yu, R. T., Subramaniam, N., Sherman, M. H., Wilson, C., Rao, R., . . Evans, R. M. (2013). A vitamin D receptor/SMAD genomic circuit gates hepatic fibrotic response. Cell, 153(3), 601-613. doi:10.1016/j.cell.2013.03.028

Drake, M. T., Maurer, M. J., Link, B. K., Habermann, T. M., Ansell, S. M., Micallef, I. N., . . Cerhan, J. R. (2010). Vitamin D insufficiency and prognosis in nonHodgkin's lymphoma. J Clin Oncol, 28(27), 4191-4198. doi:10.1200/JCO.2010.28.6674

Endres, B., \& DeLuca, H. F. (2001). 26-Hydroxylation of 1alpha,25-dihydroxyvitamin D3 does not occur under physiological conditions. Arch Biochem Biophys, 388(1), 127-134. doi:10.1006/abbi.2000.2271

Feldman, D., Krishnan, A. V., Swami, S., Giovannucci, E., \& Feldman, B. J. (2014). The role of vitamin D in reducing cancer risk and progression. Nat Rev Cancer, 14(5), 342-357. doi:10.1038/nrc3691

Foa, R., Vitale, A., Vignetti, M., Meloni, G., Guarini, A., De Propris, M. S., . . Party, G. A. L. W. (2011). Dasatinib as first-line treatment for adult patients with Philadelphia chromosome-positive acute lymphoblastic leukemia. Blood, 118(25), 6521-6528. doi:10.1182/blood-2011-05-351403

Fujisaki, J., Wu, J., Carlson, A. L., Silberstein, L., Putheti, P., Larocca, R., . . Lin, C. P. (2011). In vivo imaging of Treg cells providing immune privilege to the haematopoietic stem-cell niche. Nature, 474(7350), 216-219. doi:10.1038/nature10160 
Furge, L. L., \& Guengerich, F. P. (2006). Cytochrome P450 enzymes in drug metabolism and chemical toxicology: An introduction. Biochem Mol Biol Educ, 34(2), 66-74. doi:10.1002/bmb.2006.49403402066

Furmanski, B. D., Hu, S., Fujita, K. I., Li, L., Gibson, A. A., Janke, L. J., . . Baker, S. D. (2013). Contribution of ABCC4-mediated gastric transport to the absorption and efficacy of dasatinib. Clin Cancer Res, 19(16), 4359-4370. doi:10.1158/10780432.CCR-13-0980

Gao, C., Liao, M. Z., Han, L. W., Thummel, K. E., \& Mao, Q. (2018). Hepatic Transport of 25-Hydroxyvitamin D3 Conjugates: A Mechanism of 25-Hydroxyvitamin D3 Delivery to the Intestinal Tract. Drug Metab Dispos, 46(5), 581-591. doi:10.1124/dmd.117.078881

Gascon-Barre, M., Demers, C., Mirshahi, A., Neron, S., Zalzal, S., \& Nanci, A. (2003). The normal liver harbors the vitamin D nuclear receptor in nonparenchymal and biliary epithelial cells. Hepatology, 37(5), 1034-1042. doi:10.1053/jhep.2003.50176

Gawlik, A., Gepstein, V., Rozen, N., Dahan, A., Ben-Yosef, D., Wildbaum, G., . . . Tiosano, D. (2015). Duodenal Expression of 25 Hydroxyvitamin D3-1alphahydroxylase Is Higher in Adolescents Than in Children and Adults. J Clin Endocrinol Metab, 100(10), 3668-3675. doi:10.1210/jc.2015-1483

Gentile, D. M. (1996). Dexamethasone metabolism by human liver in vitro. Metabolite identification and inhibition of 6-hydroxylation. J Pharmacol Exp Ther, 277(1), 105-112. Retrieved from https://www.ncbi.nlm.nih.gov/pubmed/8613906

Glorieux, F. H., \& Pettifor, J. M. (2014). Vitamin D/dietary calcium deficiency rickets and pseudo-vitamin D deficiency rickets. Bonekey Rep, 3, 524. doi:10.1038/bonekey.2014.19

Gocek, E., Baurska, H., Marchwicka, A., \& Marcinkowska, E. (2012). Regulation of Leukemic Cell Differentiation through the Vitamin D Receptor at the Levels of Intracellular Signal Transduction, Gene Transcription, and Protein Trafficking and Stability. Leuk Res Treatment, 2012, 713243. doi:10.1155/2012/713243

Gonzalez, F. J., Liu, S. Y., \& Yano, M. (1993). Regulation of cytochrome P450 genes: molecular mechanisms. Pharmacogenetics, 3(1), 51-57. doi:10.1097/00008571199302000-00006

Goodwin, B., Hodgson, E., \& Liddle, C. (1999). The orphan human pregnane X receptor mediates the transcriptional activation of CYP3A4 by rifampicin through a distal enhancer module. Mol Pharmacol, 56(6), 1329-1339. doi:10.1124/mol.56.6.1329

Gorski, J. C., Jones, D. R., Haehner-Daniels, B. D., Hamman, M. A., O'Mara, E. M., Jr., \& Hall, S. D. (1998). The contribution of intestinal and hepatic CYP3A to the interaction between midazolam and clarithromycin. Clin Pharmacol Ther, 64(2), 133-143. doi:10.1016/S0009-9236(98)90146-1

Granvil, C. P., Yu, A. M., Elizondo, G., Akiyama, T. E., Cheung, C., Feigenbaum, L., . . . Gonzalez, F. J. (2003). Expression of the human CYP3A4 gene in the small intestine of transgenic mice: In vitro metabolism and pharmacokinetics of midazolam. Drug Metabolism and Disposition, 31(5), 548-558. doi:DOI 10.1124/dmd.31.5.548 
Greer, F. R. (2009). Defining vitamin D deficiency in children: beyond 25-OH vitamin D serum concentrations. Pediatrics, 124(5), 1471-1473. doi:10.1542/peds.20092307

Guengerich, F. P. (2008). Cytochrome p450 and chemical toxicology. Chem Res Toxicol, 21(1), 70-83. doi:10.1021/tx700079z

Haussler, M. R., Whitfield, G. K., Haussler, C. A., Hsieh, J. C., Thompson, P. D., Selznick, S. H., . . Jurutka, P. W. (1998). The nuclear vitamin D receptor: biological and molecular regulatory properties revealed. J Bone Miner Res, 13(3), 325-349. doi:10.1359/jbmr.1998.13.3.325

Haussler, M. R., Whitfield, G. K., Kaneko, I., Haussler, C. A., Hsieh, D., Hsieh, J. C., \& Jurutka, P. W. (2013). Molecular mechanisms of vitamin D action. Calcif Tissue Int, 92(2), 77-98. doi:10.1007/s00223-012-9619-0

Heaney, R. P. (2005). The Vitamin D requirement in health and disease. J Steroid Biochem Mol Biol, 97(1-2), 13-19. doi:10.1016/j.jsbmb.2005.06.020

Helou, M., Ning, Y., Yang, S., Irvine, P., Bachmann, L. M., Godder, K., \& Massey, G. (2014). Vitamin d deficiency in children with cancer. J Pediatr Hematol Oncol, 36(3), 212-217. doi:10.1097/MPH.0b013e31829f3754

Henry, H. L., \& Norman, A. W. (1984). Vitamin D: metabolism and biological actions. Annu Rev Nutr, 4, 493-520. doi:10.1146/annurev.nu.04.070184.002425

Hewison, M., Burke, F., Evans, K. N., Lammas, D. A., Sansom, D. M., Liu, P., . . . Adams, J. S. (2007). Extra-renal 25-hydroxyvitamin D3-1alpha-hydroxylase in human health and disease. J Steroid Biochem Mol Biol, 103(3-5), 316-321. doi:10.1016/j.jsbmb.2006.12.078

Hiwase, D. K., Saunders, V., Hewett, D., Frede, A., Zrim, S., Dang, P., . . White, D. L. (2008). Dasatinib cellular uptake and efflux in chronic myeloid leukemia cells: therapeutic implications. Clin Cancer Res, 14(12), 3881-3888. doi:10.1158/10780432.CCR-07-5095

Hobaus, J., Thiem, U., Hummel, D. M., \& Kallay, E. (2013). Role of calcium, vitamin D, and the extrarenal vitamin D hydroxylases in carcinogenesis. Anticancer Agents Med Chem, 13(1), 20-35. Retrieved from https://www.ncbi.nlm.nih.gov/pubmed/23094918

Holick, M. F. (2007). Vitamin D deficiency. N Engl J Med, 357(3), 266-281. doi:10.1056/NEJMra070553

Holick, M. F., Binkley, N. C., Bischoff-Ferrari, H. A., Gordon, C. M., Hanley, D. A., Heaney, R. P., . . Endocrine, S. (2011). Evaluation, treatment, and prevention of vitamin D deficiency: an Endocrine Society clinical practice guideline. J Clin Endocrinol Metab, 96(7), 1911-1930. doi:10.1210/jc.2011-0385

Holick, M. F., \& Chen, T. C. (2008). Vitamin D deficiency: a worldwide problem with health consequences. Am J Clin Nutr, 87(4), 1080S-1086S. doi:10.1093/ajcn/87.4.1080S

Honkakoski, P., \& Negishi, M. (1998). Protein serine/threonine phosphatase inhibitors suppress phenobarbital-induced Cyp2b10 gene transcription in mouse primary hepatocytes. Biochem J, 330 ( Pt 2), 889-895. doi:10.1042/bj3300889

Houlihan, D. D., Mabuchi, Y., Morikawa, S., Niibe, K., Araki, D., Suzuki, S., . . . Matsuzaki, Y. (2012). Isolation of mouse mesenchymal stem cells on the basis of 
expression of Sca-1 and PDGFR-alpha. Nat Protoc, 7(12), 2103-2111.

doi:10.1038/nprot.2012.125

Inouye, K., \& Sakaki, T. (2001). Enzymatic studies on the key enzymes of vitamin D metabolism; 1 alpha-hydroxylase (CYP27B1) and 24-hydroxylase (CYP24).

Biotechnol Апnи Rev, 7, 179-194. doi:10.1016/s1387-2656(01)07037-5

Iwamoto, S., Mihara, K., Downing, J. R., Pui, C. H., \& Campana, D. (2007).

Mesenchymal cells regulate the response of acute lymphoblastic leukemia cells to asparaginase. J Clin Invest, 117(4), 1049-1057. doi:10.1172/JCI30235

Johnson, E. J., Won, C. S., Kock, K., \& Paine, M. F. (2017). Prioritizing pharmacokinetic drug interaction precipitants in natural products: application to OATP inhibitors in grapefruit juice. Biopharm Drug Dispos, 38(3), 251-259. doi:10.1002/bdd.2061

Jones, B. G., Oshansky, C. M., Bajracharya, R., Tang, L., Sun, Y., Wong, S. S., . . . Hurwitz, J. L. (2016). Retinol binding protein and vitamin D associations with serum antibody isotypes, serum influenza virus-specific neutralizing activities and airway cytokine profiles. Clin Exp Immunol, 183(2), 239-247.

doi: $10.1111 /$ cei.12718

Jones, G., Strugnell, S. A., \& DeLuca, H. F. (1998). Current understanding of the molecular actions of vitamin D. Physiol Rev, 78(4), 1193-1231. doi:10.1152/physrev.1998.78.4.1193

Juarez, J., Dela Pena, A., Baraz, R., Hewson, J., Khoo, M., Cisterne, A., . . Bendall, L. J. (2007). CXCR4 antagonists mobilize childhood acute lymphoblastic leukemia cells into the peripheral blood and inhibit engraftment. Leukemia, 21(6), 12491257. doi:10.1038/sj.leu.2404684

Kagi, L., Bettoni, C., Pastor-Arroyo, E. M., Schnitzbauer, U., Hernando, N., \& Wagner, C. A. (2018). Regulation of vitamin D metabolizing enzymes in murine renal and extrarenal tissues by dietary phosphate, FGF23, and 1,25(OH)2D3. PLoS One, 13(5), e0195427. doi:10.1371/journal.pone.0195427

Kamath, A. V., Wang, J., Lee, F. Y., \& Marathe, P. H. (2008). Preclinical pharmacokinetics and in vitro metabolism of dasatinib (BMS-354825): a potent oral multi-targeted kinase inhibitor against SRC and BCR-ABL. Cancer Chemother Pharmacol, 61(3), 365-376. doi:10.1007/s00280-007-0478-8

Kaminsky, L. S., \& Fasco, M. J. (1991). Small intestinal cytochromes P450. Crit Rev Toxicol, 21(6), 407-422. doi:10.3109/10408449209089881

Kim, M., Mirandola, L., Pandey, A., Nguyen, D. D., Jenkins, M. R., Turcel, M., . . . Chiriva-Internati, M. (2012). Application of vitamin D and derivatives in hematological malignancies. Cancer Lett, 319(1), 8-22. doi:10.1016/j.canlet.2011.10.026

Kliewer, S. A., Moore, J. T., Wade, L., Staudinger, J. L., Watson, M. A., Jones, S. A., . . Lehmann, J. M. (1998). An orphan nuclear receptor activated by pregnanes defines a novel steroid signaling pathway. Cell, 92(1), 73-82. doi:10.1016/s00928674(00)80900-9

Kolars, J. C., Awni, W. M., Merion, R. M., \& Watkins, P. B. (1991). First-pass metabolism of cyclosporin by the gut. Lancet, 338(8781), 1488-1490. doi:10.1016/0140-6736(91)92302-i 
Kolars, J. C., Lown, K. S., Schmiedlin-Ren, P., Ghosh, M., Fang, C., Wrighton, S. A., . . . Watkins, P. B. (1994). CYP3A gene expression in human gut epithelium. Pharmacogenetics, 4(5), 247-259. doi:10.1097/00008571-199410000-00003

Krebsbach, P. H., Kuznetsov, S. A., Bianco, P., \& Robey, P. G. (1999). Bone marrow stromal cells: characterization and clinical application. Crit Rev Oral Biol Med, 10(2), 165-181. doi:10.1177/10454411990100020401

Kuhl, S., Gotz, H., Brochhausen, C., Jakse, N., Filippi, A., d'Hoedt, B., \& Kreisler, M. (2012). The influence of substitute materials on bone density after maxillary sinus augmentation: a microcomputed tomography study. Int J Oral Maxillofac Implants, 27(6), 1541-1546. Retrieved from https://www.ncbi.nlm.nih.gov/pubmed/23189308

Kumar, B., Garcia, M., Weng, L., Jung, X., Murakami, J. L., Hu, X., . . . Chen, C. C. (2018). Acute myeloid leukemia transforms the bone marrow niche into a leukemia-permissive microenvironment through exosome secretion. Leukemia, 32(3), 575-587. doi:10.1038/leu.2017.259

Kumar, J., Muntner, P., Kaskel, F. J., Hailpern, S. M., \& Melamed, M. L. (2009). Prevalence and associations of 25-hydroxyvitamin D deficiency in US children: NHANES 2001-2004. Pediatrics, 124(3), e362-370. doi:10.1542/peds.2009-0051

Kumar, R. (1984). Metabolism of 1,25-dihydroxyvitamin D3. Physiol Rev, 64(2), 478504. doi:10.1152/physrev.1984.64.2.478

Ladas, E. J., Orjuela, M., Stevenson, K., Cole, P. D., Lin, M., Athale, U. H., . . Kelly, K. M. (2016). Dietary intake and childhood leukemia: The Diet and Acute Lymphoblastic Leukemia Treatment (DALLT) cohort study. Nutrition, 32(10), 1103-1109 e1101. doi:10.1016/j.nut.2016.03.014

Lagas, J. S., van Waterschoot, R. A., van Tilburg, V. A., Hillebrand, M. J., Lankheet, N., Rosing, H., . . . Schinkel, A. H. (2009). Brain accumulation of dasatinib is restricted by $\mathrm{P}$-glycoprotein $(\mathrm{ABCB} 1)$ and breast cancer resistance protein (ABCG2) and can be enhanced by elacridar treatment. Clin Cancer Res, 15(7), 2344-2351. doi:10.1158/1078-0432.CCR-08-2253

Lee, H. J., Muindi, J. R., Tan, W., Hu, Q., Wang, D., Liu, S., . . Wetzler, M. (2014). Low 25(OH) vitamin D3 levels are associated with adverse outcome in newly diagnosed, intensively treated adult acute myeloid leukemia. Cancer, 120(4), 521529. doi:10.1002/cncr.28368

Lee, J. Y., So, T. Y., \& Thackray, J. (2013). A review on vitamin d deficiency treatment in pediatric patients. J Pediatr Pharmacol Ther, 18(4), 277-291. doi:10.5863/1551-6776-18.4.277

Li, J., Chen, R., Yao, Q. Y., Liu, S. J., Tian, X. Y., Hao, C. Y., . . Zhou, T. Y. (2018). Time-dependent pharmacokinetics of dexamethasone and its efficacy in human breast cancer xenograft mice: a semi-mechanism-based pharmacokinetic/pharmacodynamic model. Acta Pharmacol Sin, 39(3), 472-481. doi:10.1038/aps.2017.153

Li, X., He, Y., Ruiz, C. H., Koenig, M., Cameron, M. D., \& Vojkovsky, T. (2009). Characterization of dasatinib and its structural analogs as CYP3A4 mechanismbased inactivators and the proposed bioactivation pathways. Drug Metab Dispos, 37(6), 1242-1250. doi:10.1124/dmd.108.025932 
Lin, J. H. (2006). CYP induction-mediated drug interactions: in vitro assessment and clinical implications. Pharm Res, 23(6), 1089-1116. doi:10.1007/s11095-0060277-7

Lindh, J. D. (2011). Seasonal variation in blood drug concentrations and a potential relationship to vitamin D. Drug Metab Dispos, 39(5), 933-937.

doi:10.1124/dmd.111.038125

Lips, P., Gielen, E., \& van Schoor, N. M. (2014). Vitamin D supplements with or without calcium to prevent fractures. Bonekey Rep, 3, 512. doi:10.1038/bonekey.2014.7

Liu, P., Oyajobi, B. O., Russell, R. G., \& Scutt, A. (1999). Regulation of osteogenic differentiation of human bone marrow stromal cells: interaction between transforming growth factor-beta and 1,25(OH)(2) vitamin $\mathrm{D}(3)$ In vitro. Calcif Tissue Int, 65(2), 173-180. doi:10.1007/s002239900678

Liu, Y. T., Hao, H. P., Liu, C. X., Wang, G. J., \& Xie, H. G. (2007). Drugs as CYP3A probes, inducers, and inhibitors. Drug Metab Rev, 39(4), 699-721. doi:10.1080/03602530701690374

Loew, D., Schuster, O., \& Graul, E. H. (1986). Dose-dependent pharmacokinetics of dexamethasone. Eur J Clin Pharmacol, 30(2), 225-230. doi:10.1007/BF00614309

Lown, K. S., Kolars, J. C., Thummel, K. E., Barnett, J. L., Kunze, K. L., Wrighton, S. A., \& Watkins, P. B. (1994). Interpatient heterogeneity in expression of CYP3A4 and CYP3A5 in small bowel. Lack of prediction by the erythromycin breath test.

Drug Metab Dispos, 22(6), 947-955. Retrieved from https://www.ncbi.nlm.nih.gov/pubmed/7895614

Luczynska, A., Kaaks, R., Rohrmann, S., Becker, S., Linseisen, J., Buijsse, B., . . . Nieters, A. (2013). Plasma 25-hydroxyvitamin D concentration and lymphoma risk: results of the European Prospective Investigation into Cancer and Nutrition. Am J Clin Nutr, 98(3), 827-838. doi:10.3945/ajcn.112.054676

Luo, F. R., Yang, Z., Camuso, A., Smykla, R., McGlinchey, K., Fager, K., . . Lee, F. Y. (2006). Dasatinib (BMS-354825) pharmacokinetics and pharmacodynamic biomarkers in animal models predict optimal clinical exposure. Clin Cancer Res, 12(23), 7180-7186. doi:10.1158/1078-0432.CCR-06-1112

Mackowiak, B., \& Wang, H. (2016). Mechanisms of xenobiotic receptor activation: Direct vs. indirect. Biochim Biophys Acta, 1859(9), 1130-1140. doi:10.1016/j.bbagrm.2016.02.006

Madhusoodhan, P. P., Carroll, W. L., \& Bhatla, T. (2016). Progress and Prospects in Pediatric Leukemia. Curr Probl Pediatr Adolesc Health Care, 46(7), 229-241. doi:10.1016/j.cppeds.2016.04.003

Makishima, M., Lu, T. T., Xie, W., Whitfield, G. K., Domoto, H., Evans, R. M., . . Mangelsdorf, D. J. (2002). Vitamin D receptor as an intestinal bile acid sensor. Science, 296(5571), 1313-1316. doi:10.1126/science.1070477

Mao, Q., \& Unadkat, J. D. (2015). Role of the breast cancer resistance protein (BCRP/ABCG2) in drug transport--an update. AAPS J, 17(1), 65-82. doi:10.1208/s12248-014-9668-6

Margolin JF, S. C., Poplack DG. (2006). Principles and practice of pediatric oncology. Lippincott Williams \& Wilkins, p. 538.

Matsubara, T., Yoshinari, K., Aoyama, K., Sugawara, M., Sekiya, Y., Nagata, K., \& Yamazoe, Y. (2008). Role of vitamin D receptor in the lithocholic acid-mediated 
CYP3A induction in vitro and in vivo. Drug Metab Dispos, 36(10), 2058-2063. doi:10.1124/dmd.108.021501

Matsunaga, T., Maruyama, M., Matsubara, T., Nagata, K., Yamazoe, Y., \& Ohmori, S. (2012). Mechanisms of CYP3A induction by glucocorticoids in human fetal liver cells. Drug Metab Pharmacokinet, 27(6), 653-657. doi:10.2133/dmpk.dmpk-12nt-018

Mazziotti, G., Giustina, A., Canalis, E., \& Bilezikian, J. P. (2007). Glucocorticoidinduced osteoporosis: clinical and therapeutic aspects. Arq Bras Endocrinol Metabol, 51(8), 1404-1412. Retrieved from https://www.ncbi.nlm.nih.gov/pubmed/18209880

McFarlane, L., Truong, V., Palmer, J. S., \& Wilhelm, D. (2013). Novel PCR assay for determining the genetic sex of mice. Sex Dev, 7(4), 207-211. doi: $10.1159 / 000348677$

Melamed, M. L., Michos, E. D., Post, W., \& Astor, B. (2008). 25-hydroxyvitamin D levels and the risk of mortality in the general population. Arch Intern Med, 168(15), 1629-1637. doi:10.1001/archinte.168.15.1629

Meyer zu Schwabedissen, H. E., \& Kroemer, H. K. (2011). In vitro and in vivo evidence for the importance of breast cancer resistance protein transporters (BCRP/MXR/ABCP/ABCG2). Handb Exp Pharmacol(201), 325-371. doi:10.1007/978-3-642-14541-4_9

Mihara, K., Imai, C., Coustan-Smith, E., Dome, J. S., Dominici, M., Vanin, E., \& Campana, D. (2003). Development and functional characterization of human bone marrow mesenchymal cells immortalized by enforced expression of telomerase. Br J Haematol, 120(5), 846-849. Retrieved from https://www.ncbi.nlm.nih.gov/pubmed/12614220

Mitchell, C. D., Richards, S. M., Kinsey, S. E., Lilleyman, J., Vora, A., Eden, T. O., \& Medical Research Council Childhood Leukaemia Working, P. (2005). Benefit of dexamethasone compared with prednisolone for childhood acute lymphoblastic leukaemia: results of the UK Medical Research Council ALL97 randomized trial. Br J Haematol, 129(6), 734-745. doi:10.1111/j.1365-2141.2005.05509.x

Mowafi, F., Cagigi, A., Matskova, L., Bjork, O., Chiodi, F., \& Nilsson, A. (2008). Chemokine CXCL12 enhances proliferation in pre-B-ALL via STAT5 activation. Pediatr Blood Cancer, 50(4), 812-817. doi:10.1002/pbc.21370

Mullighan, C. G., Miller, C. B., Radtke, I., Phillips, L. A., Dalton, J., Ma, J., . . . Downing, J. R. (2008). BCR-ABL1 lymphoblastic leukaemia is characterized by the deletion of Ikaros. Nature, 453(7191), 110-114. doi:10.1038/nature06866

Nakasone, E. S., Askautrud, H. A., Kees, T., Park, J. H., Plaks, V., Ewald, A. J., . . Egeblad, M. (2012). Imaging tumor-stroma interactions during chemotherapy reveals contributions of the microenvironment to resistance. Cancer Cell, 21(4), 488-503. doi:10.1016/j.ccr.2012.02.017

Nath, S. V., Nicholson, I., Tapp, H., Zola, H., Zannettino, A. C., \& Revesz, T. (2011). Reticulin fibres anchor leukaemic blasts in the marrow of patients with acute lymphoblastic leukaemia. Med Hypotheses, 77(3), 333-335. doi:10.1016/j.mehy.2011.05.007 
Naz, A., Qureshi, R. N., Shamsi, T. S., \& Mahboob, T. (2013). Vitamin D levels in patients of acute leukemia before and after remission-induction therapy. Pakistan Journal of Medical Sciences, 29(1), 10-14. doi:10.12669/pjms.291.2764

Naz, A., R, N. Q., T, S. S., \& Mahboob, T. (2013). Vitamin D levels in patients of acute leukemia before and after remission-induction therapy. Pak J Med Sci, 29(1), 1014. doi:10.12669/pjms.291.2764

Nebert, D. W., \& Russell, D. W. (2002). Clinical importance of the cytochromes P450. Lancet, 360(9340), 1155-1162. doi:10.1016/S0140-6736(02)11203-7

Newmark, H. L., Yang, K., Kurihara, N., Fan, K., Augenlicht, L. H., \& Lipkin, M. (2009). Western-style diet-induced colonic tumors and their modulation by calcium and vitamin D in C57Bl/6 mice: a preclinical model for human sporadic colon cancer. Carcinogenesis, 30(1), 88-92. doi:10.1093/carcin/bgn229

Nombela-Arrieta, C., Pivarnik, G., Winkel, B., Canty, K. J., Harley, B., Mahoney, J. E., . . Silberstein, L. E. (2013). Quantitative imaging of haematopoietic stem and progenitor cell localization and hypoxic status in the bone marrow microenvironment. Nat Cell Biol, 15(5), 533-543. doi:10.1038/ncb2730

Omatsu, Y., Sugiyama, T., Kohara, H., Kondoh, G., Fujii, N., Kohno, K., \& Nagasawa, T. (2010). The essential functions of adipo-osteogenic progenitors as the hematopoietic stem and progenitor cell niche. Immunity, 33(3), 387-399. doi:10.1016/j.immuni.2010.08.017

Ooi, L. L., Zhou, H., Kalak, R., Zheng, Y., Conigrave, A. D., Seibel, M. J., \& Dunstan, C. R. (2010). Vitamin D deficiency promotes human breast cancer growth in a murine model of bone metastasis. Cancer Res, 70(5), 1835-1844. doi:10.1158/0008-5472.CAN-09-3194

Orgel, E., Mueske, N. M., Sposto, R., Gilsanz, V., Wren, T. A. L., Freyer, D. R., . . Mittelman, S. D. (2017). A randomized controlled trial testing an adherenceoptimized Vitamin D regimen to mitigate bone change in adolescents being treated for acute lymphoblastic leukemia. Leuk Lymphoma, 58(10), 2370-2378. doi:10.1080/10428194.2017.1289526

Paine, M. F., Khalighi, M., Fisher, J. M., Shen, D. D., Kunze, K. L., Marsh, C. L., . . Thummel, K. E. (1997). Characterization of interintestinal and intraintestinal variations in human CYP3A-dependent metabolism. J Pharmacol Exp Ther, 283(3), 1552-1562. Retrieved from https://www.ncbi.nlm.nih.gov/pubmed/9400033

Paine, M. F., Ludington, S. S., Chen, M. L., Stewart, P. W., Huang, S. M., \& Watkins, P. B. (2005). Do men and women differ in proximal small intestinal CYP3A or Pglycoprotein expression? Drug Metab Dispos, 33(3), 426-433. doi:10.1124/dmd.104.002469

Pavek, P. (2016). Pregnane X Receptor (PXR)-Mediated Gene Repression and CrossTalk of PXR with Other Nuclear Receptors via Coactivator Interactions. Front Pharmacol, 7, 456. doi:10.3389/fphar.2016.00456

Pavek, P., \& Dvorak, Z. (2008). Xenobiotic-induced transcriptional regulation of xenobiotic metabolizing enzymes of the cytochrome P450 superfamily in human extrahepatic tissues. Curr Drug Metab, 9(2), 129-143.

doi:10.2174/138920008783571774 
Peled, A., Petit, I., Kollet, O., Magid, M., Ponomaryov, T., Byk, T., . . . Lapidot, T. (1999). Dependence of human stem cell engraftment and repopulation of NOD/SCID mice on CXCR4. Science, 283(5403), 845-848. doi:10.1126/science.283.5403.845

Peltoniemi, M. A., Saari, T. I., Hagelberg, N. M., Laine, K., Neuvonen, P. J., \& Olkkola, K. T. (2012). St John's wort greatly decreases the plasma concentrations of oral Sketamine. Fundam Clin Pharmacol, 26(6), 743-750. doi:10.1111/j.14728206.2011.00954.x

Podzolkov, V. I., Pokrovskaya, A. E., \& Panasenko, O. I. (2018). Vitamin D deficiency and cardiovascular pathology. Ter Arkh, 90(9), 144-150. doi:10.26442/terarkh2018909144-150

Prentice, A. (2013). Nutritional rickets around the world. J Steroid Biochem Mol Biol, 136, 201-206. doi:10.1016/j.jsbmb.2012.11.018

Prockop, D. J. (1997). Marrow stromal cells as stem cells for nonhematopoietic tissues. Science, 276(5309), 71-74. doi:10.1126/science.276.5309.71

Quach, H. P., Noh, K., Hoi, S. Y., Bruinsma, A., Groothuis, G. M. M., Li, A. P., . . Pang, K. S. (2018). Alterations in gene expression in vitamin D-deficiency: Down-regulation of liver Cyp7a1 and renal Oat3 in mice. Biopharm Drug Dispos, 39(2), 99-115. doi:10.1002/bdd.2118

Ramsey, L. B., Janke, L., Payton, M. A., Cai, X. J., Jeha, S., Pui, C. H., . . Relling, M. V. (2014). Antileukemic Efficacy of Continuous Vs Discontinuous Dexamethasone in Murine Xenografts of Acute Lymphoblastic Leukemia. Blood, 124(21). Retrieved from <Go to ISI >://WOS:000349242702066

Ramsey, L. B., Janke, L. J., Payton, M. A., Cai, X., Paugh, S. W., Karol, S. E., . . Relling, M. V. (2015). Antileukemic Efficacy of Continuous vs Discontinuous Dexamethasone in Murine Models of Acute Lymphoblastic Leukemia. PLoS One, 10(8), e0135134. doi:10.1371/journal.pone.0135134

Ray, R., Banks, M., Abuzahra, H., Eddy, V. J., Persons, K. S., Lucia, M. S., . . Holick, M. F. (2012). Effect of dietary vitamin D and calcium on the growth of androgeninsensitive human prostate tumor in a murine model. Anticancer Res, 32(3), 727731. Retrieved from https://www.ncbi.nlm.nih.gov/pubmed/22399584

Reid, I. R. (1997). Glucocorticoid osteoporosis--mechanisms and management. Eur J Endocrinol, 137(3), 209-217. doi:10.1530/eje.0.1370209

Renaud, H. J., Cui, J. Y., Khan, M., \& Klaassen, C. D. (2011). Tissue distribution and gender-divergent expression of 78 cytochrome P450 mRNAs in mice. Toxicol Sci, 124(2), 261-277. doi:10.1093/toxsci/kfr240

Reschly, E. J., \& Krasowski, M. D. (2006). Evolution and function of the NR1I nuclear hormone receptor subfamily (VDR, PXR, and CAR) with respect to metabolism of xenobiotics and endogenous compounds. Curr Drug Metab, 7(4), 349-365. doi:10.2174/138920006776873526

Russel, F. G., Koenderink, J. B., \& Masereeuw, R. (2008). Multidrug resistance protein 4 (MRP4/ABCC4): a versatile efflux transporter for drugs and signalling molecules. Trends Pharmacol Sci, 29(4), 200-207. doi:10.1016/j.tips.2008.01.006

Sahay, M., \& Sahay, R. (2012). Rickets-vitamin D deficiency and dependency. Indian J Endocrinol Metab, 16(2), 164-176. doi:10.4103/2230-8210.93732 
Schafer, A. L., Weaver, C. M., Black, D. M., Wheeler, A. L., Chang, H., Szefc, G. V., . . . Sellmeyer, D. E. (2015). Intestinal Calcium Absorption Decreases Dramatically After Gastric Bypass Surgery Despite Optimization of Vitamin D Status. J Bone Miner Res, 30(8), 1377-1385. doi:10.1002/jbmr.2467

Schinkel, A. H., Wagenaar, E., van Deemter, L., Mol, C. A., \& Borst, P. (1995). Absence of the mdr1a P-Glycoprotein in mice affects tissue distribution and pharmacokinetics of dexamethasone, digoxin, and cyclosporin A. J Clin Invest, 96(4), 1698-1705. doi:10.1172/JCI118214

Schuetz, E. G., Schmid, W., Schutz, G., Brimer, C., Yasuda, K., Kamataki, T., . . Cole, T. J. (2000). The glucocorticoid receptor is essential for induction of cytochrome P-4502B by steroids but not for drug or steroid induction of CYP3A or P-450 reductase in mouse liver. Drug Metab Dispos, 28(3), 268-278. Retrieved from https://www.ncbi.nlm.nih.gov/pubmed/10681370

Schuetz, E. G., Wrighton, S. A., Barwick, J. L., \& Guzelian, P. S. (1984). Induction of cytochrome P-450 by glucocorticoids in rat liver. I. Evidence that glucocorticoids and pregnenolone 16 alpha-carbonitrile regulate de novo synthesis of a common form of cytochrome P-450 in cultures of adult rat hepatocytes and in the liver in vivo. J Biol Chem, 259(3), 1999-2006. Retrieved from https://www.ncbi.nlm.nih.gov/pubmed/6141167

Schuetz, E. G., Wrighton, S. A., Safe, S. H., \& Guzelian, P. S. (1986). Regulation of cytochrome P-450p by phenobarbital and phenobarbital-like inducers in adult rat hepatocytes in primary monolayer culture and in vivo. Biochemistry, 25(5), 11241133. doi:10.1021/bi00353a027

Schwartz, J. B. (2009). Effects of vitamin D supplementation in atorvastatin-treated patients: a new drug interaction with an unexpected consequence. Clin Pharmacol Ther, 85(2), 198-203. doi:10.1038/clpt.2008.165

Seyedalipour, F., Mansouri, A., Vaezi, M., Gholami, K., Heidari, K., Hadjibabaie, M., \& Ghavamzadeh, A. (2017). High Prevalence of Vitamin D Deficiency in Newly Diagnosed Acute Myeloid Leukemia Patients and Its Adverse Outcome. Int J Hematol Oncol Stem Cell Res, 11(3), 209-216. Retrieved from https://www.ncbi.nlm.nih.gov/pubmed/28989587

Shanafelt, T. D., Drake, M. T., Maurer, M. J., Allmer, C., Rabe, K. G., Slager, S. L., . . . Cerhan, J. R. (2011). Vitamin D insufficiency and prognosis in chronic lymphocytic leukemia. Blood, 117(5), 1492-1498. doi:10.1182/blood-2010-07295683

Sherman, M. H., Yu, R. T., Engle, D. D., Ding, N., Atkins, A. R., Tiriac, H., . . Evans, R. M. (2014). Vitamin D receptor-mediated stromal reprogramming suppresses pancreatitis and enhances pancreatic cancer therapy. Cell, 159(1), 80-93. doi:10.1016/j.cell.2014.08.007

Siebe, H., Baude, G., Lichtenstein, I., Wang, D., Buhler, H., Hoyer, G. A., \& Hierholzer, K. (1993). Metabolism of dexamethasone: sites and activity in mammalian tissues. Ren Physiol Biochem, 16(1-2), 79-88. doi:10.1159/000173753

Sinha, A., Avery, P., Turner, S., Bailey, S., \& Cheetham, T. (2011). Vitamin D status in paediatric patients with cancer. Pediatr Blood Cancer, 57(4), 594-598. doi:10.1002/pbc. 22963 
Sipkins, D. A., Wei, X., Wu, J. W., Runnels, J. M., Cote, D., Means, T. K., . . . Lin, C. P. (2005). In vivo imaging of specialized bone marrow endothelial microdomains for tumour engraftment. Nature, 435(7044), 969-973. doi:10.1038/nature03703

Squibb, B.-M. (2006). Sprycel: Summary of product characteristics. https://www.ema.europa.eu/en/documents/product-information/sprycel-eparproduct-information_en.pdf.

Stacy, A. E., Jansson, P. J., \& Richardson, D. R. (2013). Molecular pharmacology of ABCG2 and its role in chemoresistance. Mol Pharmacol, 84(5), 655-669. doi:10.1124/mol.113.088609

Strahlendorf, C., Pole, J., Barber, R. (2018). Enrolling children with acute lymphoblastic leukaemia on a clinical trial improves event-free survival: a population-based study. Br J Cancer, 118. doi:https://doi.org/10.1038/bjc.2017.462

Strolin Benedetti, M., \& Dostert, P. (1994). Induction and autoinduction properties of rifamycin derivatives: a review of animal and human studies. Environ Health Perspect, 102 Suppl 9, 101-105. doi:10.1289/ehp.94102s9101

Strom, S. C., Pisarov, L. A., Dorko, K., Thompson, M. T., Schuetz, J. D., \& Schuetz, E. G. (1996). Use of human hepatocytes to study P450 gene induction. Methods Enzymol, 272, 388-401. doi:10.1016/s0076-6879(96)72044-X

Swami, S., Krishnan, A. V., Wang, J. Y., Jensen, K., Horst, R., Albertelli, M. A., \& Feldman, D. (2012). Dietary vitamin D(3) and 1,25-dihydroxyvitamin D(3) (calcitriol) exhibit equivalent anticancer activity in mouse xenograft models of breast and prostate cancer. Endocrinology, 153(6), 2576-2587. doi:10.1210/en.2011-1600

Tanaka, H., Abe, E., Miyaura, C., Kuribayashi, T., Konno, K., Nishii, Y., \& Suda, T. (1982). 1 alpha,25-Dihydroxycholecalciferol and a human myeloid leukaemia cell line (HL-60). Biochem J, 204(3), 713-719. doi:10.1042/bj2040713

Tangpricha, V., Spina, C., Yao, M., Chen, T. C., Wolfe, M. M., \& Holick, M. F. (2005). Vitamin D deficiency enhances the growth of MC-26 colon cancer xenografts in Balb/c mice. J Nutr, 135(10), 2350-2354. doi:10.1093/jn/135.10.2350

Tenen, D. G. (2003). Disruption of differentiation in human cancer: AML shows the way. Nat Rev Cancer, 3(2), 89-101. doi:10.1038/nrc989

Thirumaran, R. K., Lamba, J. K., Kim, R. B., Urquhart, B. L., Gregor, J. C., Chande, N., . .. Schuetz, E. G. (2012). Intestinal CYP3A4 and midazolam disposition in vivo associate with VDR polymorphisms and show seasonal variation. Biochemical Pharmacology, 84(1), 104-112. doi:10.1016/j.bcp.2012.03.017

Thummel, K. E., Brimer, C., Yasuda, K., Thottassery, J., Senn, T., Lin, Y., . . Schuetz, E. (2001). Transcriptional control of intestinal cytochrome P-4503A by 1alpha,25-dihydroxy vitamin D3. Mol Pharmacol, 60(6), 1399-1406. Retrieved from https://www.ncbi.nlm.nih.gov/pubmed/11723248

Thummel, K. E., O'Shea, D., Paine, M. F., Shen, D. D., Kunze, K. L., Perkins, J. D., \& Wilkinson, G. R. (1996). Oral first-pass elimination of midazolam involves both gastrointestinal and hepatic CYP3A-mediated metabolism. Clin Pharmacol Ther, 59(5), 491-502. doi:10.1016/S0009-9236(96)90177-0

Tomlinson, E. S., Lewis, D. F., Maggs, J. L., Kroemer, H. K., Park, B. K., \& Back, D. J. (1997). In vitro metabolism of dexamethasone (DEX) in human liver and kidney: the involvement of CYP3A4 and CYP17 (17,20 LYASE) and molecular 
modelling studies. Biochemical Pharmacology, 54(5), 605-611.

doi:10.1016/s0006-2952(97)00166-4

Tredger, J. M., Smith, H. M., Powell-Jackson, P. R., Davis, M., \& Williams, R. (1981).

Effect of rifampicin on the mouse hepatic mixed-function oxidase system.

Biochemical Pharmacology, 30(10), 1043-1051. doi:10.1016/00062952(81)90440-8

Turner, R. T., Puzas, J. E., Forte, M. D., Lester, G. E., Gray, T. K., Howard, G. A., \& Baylink, D. J. (1980). In vitro synthesis of 1 alpha,25-dihydroxycholecalciferol and 24,25-dihydroxycholecalciferol by isolated calvarial cells. Proc Natl Acad Sci $U S$ A, 77(10), 5720-5724. doi:10.1073/pnas.77.10.5720

van den Berk, L. C., van der Veer, A., Willemse, M. E., Theeuwes, M. J., Luijendijk, M. W., Tong, W. H., ... den Boer, M. L. (2014). Disturbed CXCR4/CXCL12 axis in paediatric precursor B-cell acute lymphoblastic leukaemia. Br J Haematol, 166(2), 240-249. doi:10.1111/bjh.12883

Wallace, G., Jodele, S., Howell, J., Myers, K. C., Teusink, A., Zhao, X., . . Davies, S. M. (2015). Vitamin D Deficiency and Survival in Children after Hematopoietic Stem Cell Transplant. Biol Blood Marrow Transplant, 21(9), 1627-1631. doi:10.1016/j.bbmt.2015.06.009

Watkins, P. B., Wrighton, S. A., Maurel, P., Schuetz, E. G., Mendez-Picon, G., Parker, G. A., \& Guzelian, P. S. (1985). Identification of an inducible form of cytochrome P450 in human liver. Proc Natl Acad Sci U S A, 82(18), 6310-6314. doi:10.1073/pnas.82.18.6310

Williams, R. T., Roussel, M. F., \& Sherr, C. J. (2006). Arf gene loss enhances oncogenicity and limits imatinib response in mouse models of Bcr-Abl-induced acute lymphoblastic leukemia. Proc Natl Acad Sci U S A, 103(17), 6688-6693. doi:10.1073/pnas.0602030103

Williams, R. T., \& Sherr, C. J. (2008). The INK4-ARF (CDKN2A/B) locus in hematopoiesis and BCR-ABL-induced leukemias. Cold Spring Harb Symp Quant Biol, 73, 461-467. doi:10.1101/sqb.2008.73.039

Wrighton, S. A., Schuetz, E. G., Watkins, P. B., Maurel, P., Barwick, J., Bailey, B. S., . . . Guzelian, P. (1985). Demonstration in multiple species of inducible hepatic cytochromes P-450 and their mRNAs related to the glucocorticoid-inducible cytochrome P-450 of the rat. Mol Pharmacol, 28(3), 312-321. Retrieved from https://www.ncbi.nlm.nih.gov/pubmed/3875783

Xu, Y., Hashizume, T., Shuhart, M. C., Davis, C. L., Nelson, W. L., Sakaki, T., . . . Thummel, K. E. (2006). Intestinal and hepatic CYP3A4 catalyze hydroxylation of 1alpha,25-dihydroxyvitamin D(3): implications for drug-induced osteomalacia. Mol Pharmacol, 69(1), 56-65. doi:10.1124/mol.105.017392

Yanagimoto, T., Itoh, S., Sawada, M., \& Kamataki, T. (1997). Mouse cytochrome P450 (Cyp3a11): predominant expression in liver and capacity to activate aflatoxin B1. Arch Biochem Biophys, 340(2), 215-218. doi:10.1006/abbi.1997.9900

Young, J., Welin, E., Braeutigam, C., Gilger, E., Lane, A., \& Salloum, R. (2018). Impact of a Vitamin D Replacement Algorithm in Children and Young Adults With Acute Lymphoblastic Leukemia. J Pediatr Hematol Oncol, 40(8), 594-597. doi:10.1097/MPH.0000000000001204 
Yusim, A., Franklin, L., Brooke, S., Ajilore, O., \& Sapolsky, R. (2000). Glucocorticoids exacerbate the deleterious effects of gp120 in hippocampal and cortical explants. J Neurochem, 74(3), 1000-1007. doi:10.1046/j.1471-4159.2000.0741000.x

Zhang, B., Li, M., McDonald, T., Holyoake, T. L., Moon, R. T., Campana, D., . . . Bhatia, R. (2013). Microenvironmental protection of CML stem and progenitor cells from tyrosine kinase inhibitors through $\mathrm{N}$-cadherin and Wnt-beta-catenin signaling. Blood, 121(10), 1824-1838. doi:10.1182/blood-2012-02-412890

Zhang, H., LeCulyse, E., Liu, L., Hu, M., Matoney, L., Zhu, W., \& Yan, B. (1999). Rat pregnane $\mathrm{X}$ receptor: molecular cloning, tissue distribution, and xenobiotic regulation. Arch Biochem Biophys, 368(1), 14-22. doi:10.1006/abbi.1999.1307

Zhou, C., Assem, M., Tay, J. C., Watkins, P. B., Blumberg, B., Schuetz, E. G., \& Thummel, K. E. (2006). Steroid and xenobiotic receptor and vitamin D receptor crosstalk mediates CYP24 expression and drug-induced osteomalacia. J Clin Invest, 116(6), 1703-1712. doi:10.1172/JCI27793

Zhou, J. Y., Norman, A. W., Chen, D. L., Sun, G. W., Uskokovic, M., \& Koeffler, H. P. (1990). 1,25-Dihydroxy-16-ene-23-yne-vitamin D3 prolongs survival time of leukemic mice. Proc Natl Acad Sci U S A, 87(10), 3929-3932.

doi:10.1073/pnas.87.10.3929 


\section{VITA}

Kavya Annu, daughter of Manikya Reddy Annu and Vimala Pati was born in Hyderabad, India in 1992. After high school, she obtained Bachelor of Pharmacy degree from G. Pulla Reddy College of Pharmacy, Osmania University, Hyderabad, India in May 2013. She then moved to United States of America to pursue Master of Science in Pharmacology and Toxicology from Wright State University, Dayton, OH, USA and graduated in May 2015. She then worked as a Regulatory Affairs Specialist contractor with Hospira, a Pfizer company from 2015 to 2016. With keen interest in research, she joined Integrated Biomedical Sciences (IBS) program for doctoral studies at University of Tennessee Health Science Center, Memphis, TN, USA in August 2016. She expects to receive her doctorate degree in November 2020. 